\title{
Studies of Relativistic Heavy Ion Collisions \\ at the AGS (Experiment 814)
}

\author{
W. E. Cleland \\ University of Pittsburgh \\ Department of Physics and Astronomy
}

\section{DISCLAIMER}

This report was prepared as an account of work sponsored by an agency of the United States per the United States Government nor any agency thereol, nility or responsiGovernment. Neither the Uarranty, express or implied, or assumes any legal apparatus, product, or employees, makes any warkanteteness, or usefulness of any information, appaned rights. Referbility for the accuracy, completents its use would not infringe privately owe name, trademark, process disclosed, or represents that its product, process, or service by trade name, trad, recomence herein to any specific commercial product, proconstitute or imply its endorsem. manufacturer, or otherwise does not necessate Government or any agency thereof. The views mendation, or favoring by the United States not necessarily state or reflect those of the and opinions of authors expressed hercin thereof.

United States Government or any agency the

\section{Annual Progress Report}

DOE Grant NO. DE FG02 87ER40363

1 May 1991 - 30 April 1992 


\section{Studies of Relativistic Heavy Ion Collisions at the AGS (Experiment 814)}

During the past year, the Pittsburgh group has continued to work with the E814 collaboration in carrying out AGS Experiment 814. We present here a brief history of the experiment, followed by a detailed report of the analysis work being pursued at the University of Pittsburgh.

In May 1987, during the heavy ion run at the AGS, we mounted a test setup, which was used (a) to understand the behavior of the modified uranium/scintillator calorimeters, (b) to test the prototype forward scintillation counters, (c) to carry out a set of albedo measurements, and (d) to carry out a measurement of the $E_{T}$ spectrum for a $10 \mathrm{GeV} /$ nucleon beam incident upon a variety of nuclear targets. Preliminary results [1] from this run indicate that there is almost complete stopping of the ions at $10 \mathrm{GeV} /$ nucleon, as the limit in $E_{T}$ reached seems to depend little on the $A$ of the target nucleus. In November 1987, we mounted a smaller test in order to study the properties of scintillation counters in relativistic ion beams. In December 1988, the apparatus in its final configuration (but still incomplete) was exposed first to proton beams, in which debugging and testing was carried out, and then to $15 \mathrm{GeV} / \mathrm{c}^{28} \mathrm{Si}$ beams, during which a significant data sample was taken on each of the three major physics topics we are studying. The 1988 data have been analyzed, resulting in the publication of three papers $[2,3,4]$.

In June 1989, another run was carried out, in which all of the forward calorimeters were in place, while the drift chambers and the participant calorimeter were being tested. Results from this run on central collisions have been recently published [5]. Another ${ }^{28} \mathrm{Si}$ run was carried out in June 1990, in which the apparatus was finally complete, including the participant calorimeter, the drift chambers, and a new device, the beam vertex detector. There was a strong emphasis on peripheral data in this run, and a paper on $1 p$ emission has recently been submitted for publication [6](included here as Appendix 
A). Other peripheral channels are still being analyzed, and the progress in this area is one of the main topics of this report. In addition, a set of data on central collisions were taken using the silicon multiplicity detector as the trigger. The analysis of these data was the main topic of the Ph.D. thesis of M. K. Jayananda, included here as Appendix B. In addition, a paper on the charged multiplicity distribution has been submitted for publication [7]. In 1991 we carried out a calibration run on the participant calorimeter as well as a data run on central collisions, with PCAL as the main trigger. The status of the analysis of these data is described in detail below. Another topic in the 1991 run was a measurement of the $p_{T}$ spectra of forward-emitted particles, which is being analyzed by the Stony Brook group.

As originally proposed, Experiment 814 is a study of both extreme peripheral collisions and the transition from peripheral to central collisions in relativistic heavy ion-nucleus interactions. We are studying rlativistic heavy ion interactions with nuclei in two types of collisions: (a) extreme peripheral collisions of large impact parameter, and (b) central collisions with high transverse energy in the final state. The experiment emphasizes the measurement of overall event characteristics, in particular energy flow measurements and a precise measurement of the particle charge, momentum, and energy in the forward direction. This permits measurements of cross sections and rapidity densities as a function of the transverse energy for leading baryons emitted into regions of larger rapidity. Combining the energy flow measurements as a function of rapidity with the spectra of leading baryons provides information on the impact parameter dependence of the nuclear stopping of the projectile in relativistic heavy ion collisions. In 1988, the scope of Experiment 814 was enlarged to include a search for strange matter in central collisions, the first results of which have been published [4], and analysis on a longer run taken in 1990 is still under way. 


\section{Detectors in Experiment 814}

A diagram of the experiment is shown in Figure 1. We give a brief description of each of the detector systems below. More detailed information is available in our previous annual reports and in the publications and conference reports of the experiment.

- Beam vertex detector. The beam vertex detector is a pair of silicon strip detectors used to locate the horizontal position and angle of the incoming beam particle. This system was installed before the June 1990 run, in order to improve the resolution in the center of mass quantities in certain exclusive peripheral interactions.

- Beam counters. The beam detectors serve to define the presence of a beam projectile, to establish the time of the collision, and to identify the charge of the projectile. The telescope consists of two counters in coincidence and two other counters, with a hole for the beam, in anticoincidence. The geometrical coincidence signifies that a track has crossed the target. A pulse height measurement from one of the counters is used to establish the charge of the projectile, and the timing of the other counter is used to establish its time of arrival. The problems posed by large dynamic range in making good amplitude and timing measurements for scintillation counters operating in a heavy ion beam are nontrivial. Tests on the suitability of various scintillators and phototubes were carried out in a heavy ion run during the fall of 1987 . On the basis of these tests, NE102 was chosen as the scintillator material, Hammamatsu R2083 as the phototube for good timing, and EM 9954 as the tube for good charge resolution.

- Target. The target for E814 is a simple disk target with a remote positioning control. The rotating target assembly was built by the McGill group and was installed in the mount which supports the multiplicity counter. The disk has eight positions, so that up to seven targets plus an empty frame may be used at any one time. The complement of targets is changed for the different programs, as peripheral, central, and strangelet running require different materials and thicknesses. 

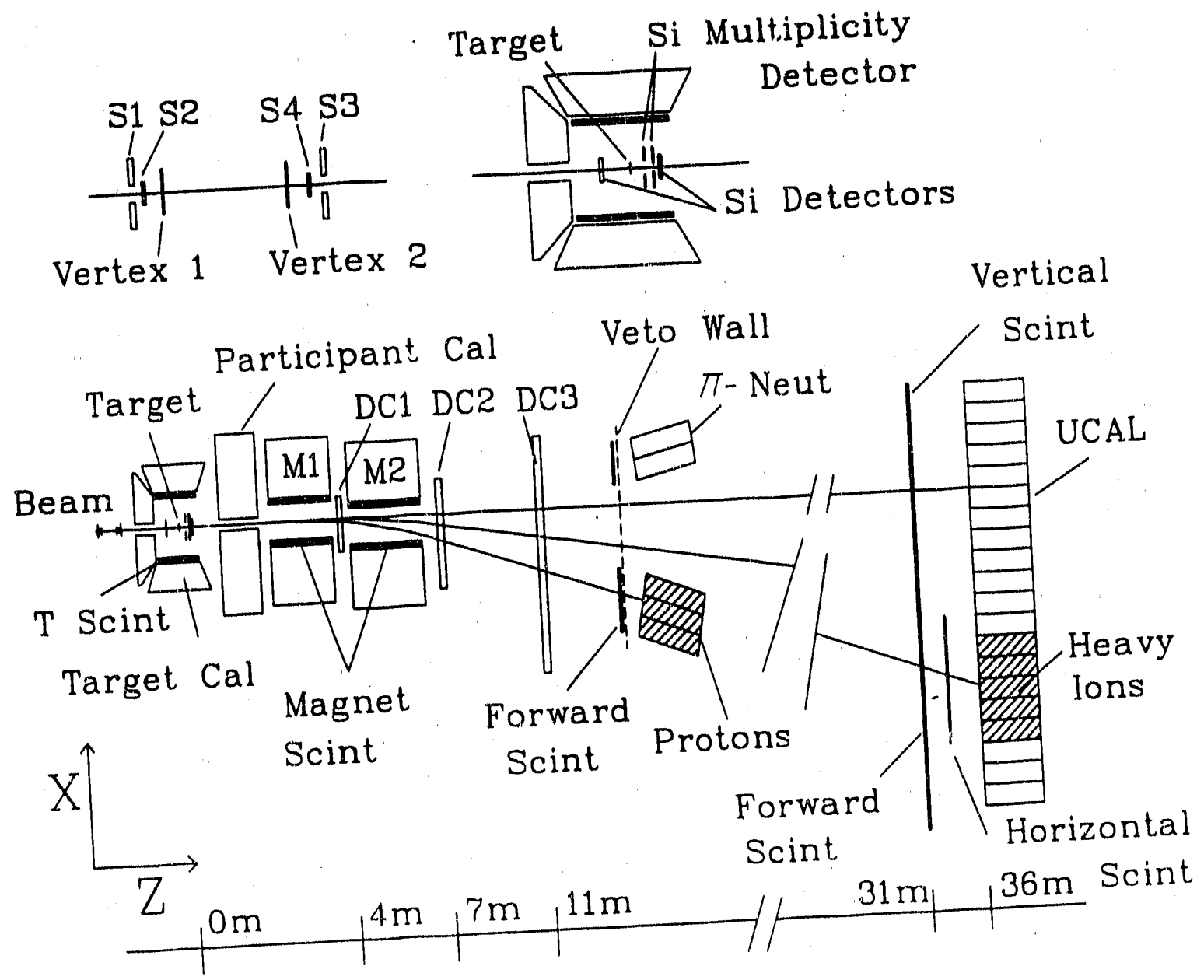

Figure 1: Diagram of the apparatus of Experiment 814. M1 and M2 are dipole magnets. DC1, $\mathrm{DC2}$, and DC3 are drift chambers used to track particles in the forward spectrometer. Solid lines in the figure represent trajectories for neutral particles, beamlike particles (with $\mathrm{Z} / \mathrm{A}=$ $1 / 2$ ) and protons with $14.6 \mathrm{GeV} / \mathrm{c}$ momentum. 
- Multiplicity Detector. The silicon multiplicity counter consists of two 512 pad silicon detectors which are positioned immediately downstream of the target. They are used both at the trigger level and in offline studies of the multiplicity as a function of rapidity. This detector was fabricated at $\mathrm{BNL}$, and the electronics was supplied by BNL, CERN, and New Mexico. K. Jayananda, a Pitt graduate student, was responsible for the pad layout and for designing the electronics for integrating it into the trigger. The detector was used successfully as a multiplicity trigger in the June 1989 run, and it was the source for most of the central triggers for the 1990 and 1991 runs. Details of the detector and analysis of the multiplicity data are given in Jayananda's Ph.D. thesis, included here as Appendix B.

- Target Calorimeter. The target calorimeter is used to detect the products of the target fragmentation and covers the region of $\theta$ between 45 and 180 degrees. It consists of a box composed of 900 blocks of NaI, $4 \times 4 \mathrm{~cm}$ in cross section and 6 radiation lengths thick. It serves to measure $E_{T}$ in central collisions and it also acts as a veto for the peripheral collisions. The $\mathrm{NaI}$ is read out using photodiodes followed by preamplifiers and shaping amplifiers. Summing electronics for the first and second level trigger has been designed and fabricated at Stony Brook. The construction and calibration of this detector is the responsibility of the Stony Brook group.

- Participant Calorimeter. The participant calorimeter, constructed by Los Alamos and Texas $A \& M$, is azimuthally symmetric, covering the polar angle region $1.6^{\circ}$ $<\theta<48^{\circ}$. It has both azimuthal and polar angle segmentation. The radiator is lead and iron plates, and the sampling layer is plastic scintillator, coupled to phototubes via wavelength-shifting fiber light guides. The electromagnetic and hadronic sections of the calorimeter are read out separately. Fast trigger sums, using $E_{T}$ weights, are formed separately for each azimuthal section, both for the electromagnetic and hadronic sections of the calorimeter. Z. Zhang, a Pitt graduate student, is responsible for the PCAL and its trigger electronics, including the problem of 
on-line and off-line gain monitoring and adjustment. A discussion of the status of the calorimeter and the data taken with the PCAL trigger is given in Section 3.2.1.

- Tracking Chambers. In order to measure the momenta of the charged particles emitted in the collisions, we use a spectrometer consisting of three sets of tracking chambers and two magnets. This configuration is chosen due to the wide range of particle momenta. One of the chambers, DC1, has highly segmented pad readout and interpolation by charge division. It is positioned between the two magnets. The chamber has 1000 pads, and the density varies across the plane in accordance with the expected track multiplicity. The design of this chamber was carried out in the Instrumentation Division of BNL. One of the key people in this group was Bo Yu, a graduate student at the University of Pittsburgh who has recently complinted his $\mathrm{Ph} . \mathrm{D}$. thesis[10], which contains several studies relevant to the E814 trackir g chambers, especially DC1. The other drift chambers for the experiment, DC2 and DC3, consist of six drift planes and two planes with cathode pad readout. They have been designed at BNL, with the fabrication of the frames carried out at Yale. These chambers were installed just before the June 1989 run and were fully operational in the June 1990 run.

- Forward Calorimeters. Charged particles with momenta greater than $6 \mathrm{GeV} / \mathrm{c}$ and all neutral particles emitted in a cone of $\theta$ less than 1 degree are measured in the forward calorimeters. In 1988, we had only 13 of these units, since they were obtained from CERN and were still required in HFiLIOS. The remaining 12 units were shipped to BNL in early 1989 and were modified and installed in the experiment before the June 1989 run, completing the set of uranium/scintillator calorimeters in the forward spectrometer. Just upstream of each of the calorimeter stacks are a bank of scintillation counters, two counters associated with each stack, which are used to measure the charge and the vertical position of the track. Because fragments emitted in the forward direction have approximately the velocity of the beam particle 15 $\mathrm{GeV} /$ nucleon, the energy measurement in the calorimeters is a good measure of the 
nucleon number. The use of calorimeters in the detection of final state neutrons is particularly attractive, as the good energy and spatial resolution of these calorimeters permit studies of correlations among leading neutrons in order to get information on transparency in nuclear collisions and to search for bound multineutron states. Thus is is important for us to understand the detailed response of these units to the forward emitted baryons.

- Scintillation Counters. There are three sets of scintillation hodoscopes used in the experiment: (a) the target counters, used in conjunction with the target calorimeter to identify charged particle fragments and neutrals; (b) magnet scintillators, which line the sidewalls of the two magnets, used to detect pions produced in the forward direction; and (c) forward scintillation counters, to be used in conjunction with the forward calorimeter to measure the charge of nuclear fragments. The target counters have been constructed and installed by the Stony Brook group. The magnet counters and forward scintillation counters have been constructed and installed by the Pitt group, and their operation and calibration is the responsibility of $U$. Sonnadara.

\section{Analysis of Peripheral Collisions}

\subsection{Data Sample}

In June 1990, during 10 days of running, we collected a large, high quality sample of data to study peripheral collisions. The recorded sample consists of two major parts, namely, (a) a single nucleon emission ( $1 \mathrm{p}-1 \mathrm{n}$ ) and (b) two or three nucleon emission $(2 \mathrm{p}-2 \mathrm{n})$. These were recorded over 6 different targets including data taken with an empty target frame. In addition, a majority of the events were recorded with an online veto requirement to enrich the data sample. A small percentage of data were taken without the online veto requirement to study trigger efficiencies. In a normal data run, the trigger was set up to record, in addition to the peripheral events, a small fraction of events with minimal trigger requirements (pre-triggers), events with no interactions (beam triggers), 
Table 1: Sample used in the analysis of peripheral data.

\begin{tabular}{|c|c|c|}
\hline Target & $\begin{array}{c}\text { No of tapes for } \\
\text { single nucleon }\end{array}$ & $\begin{array}{c}\text { No of tapes for } \\
\text { two or three nucleons }\end{array}$ \\
\hline $\mathrm{Pb}$ & 8 & 36 \\
$\mathrm{Sn}$ & 7 & 36 \\
$\mathrm{Cu}$ & 3 & 37 \\
$\mathrm{Al}$ & 5 & 35 \\
$\mathrm{C}$ & 3 & - \\
$\mathrm{MT}$ & 4 & 33 \\
\hline
\end{tabular}

and random events with no beam particles present (empty events). These were used to study gain variations and other systematic effects. The recorded data sample is shown in Table 1.

In addition we recorded 18 beam trigger tapes, 18 pre-trigger tapes and 18 pulser tapes, also used for systematic detector studies.

These data were originally written into 6250 magnetic tapes (one tape for each run). The policy of the collaboration is to keep the original copy of the data at BNL and produce copies for individual institutions onto $8 \mathrm{~mm}$ tapes which can be read via hexabyte tape drives. Each $8 \mathrm{~mm}$ tape is used to record up to 12 runs from 6250 tapes. The peripheral sample with which we are working consists of 6 (one for each target) $8 \mathrm{~mm}$ tapes for $1 \mathrm{p}-1 \mathrm{n}$ triggers and 22 tapes for $2 \mathrm{p}-2 \mathrm{n}$ or rare triggers. This sample consists of about $2,400,000$ events.

\subsection{Event Selection}

In studying peripheral interactions, the main objective is to identify pure electromagnetic interactions from nuclear induced interactions. The detectors surrounding the target region are very useful for this. The Participant Calorimeter (PCAL) is an excellent tool 
for separating these two classes. In Figure 2 we show the energy seen by the PCAL for a normal peripheral run. The peak at $8 \mathrm{GeV}$ corresponds to events where one nucleon is emitted at an angle greater than 0.8 degrees (which corresponds to the aperture in the PCAL) due to high momentum transfer from nuclear induced collisions. Pure electromagnetic events do not produce detectable energy in PCAL, so we can set un upper limit of $1.0 \mathrm{GeV}$ (a typical noise level for the total energy sum) in PCAL as a yeto against nuclear interactions.

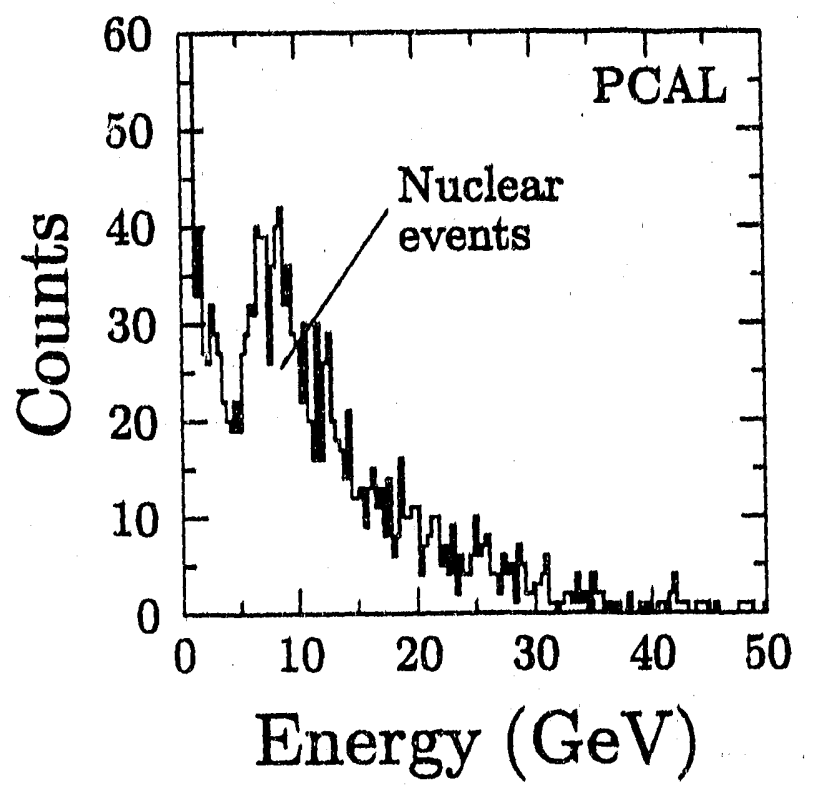

Figure 2: Energy deposited in PCAL in the peripheral data. The EMD events are charaterized by low $(<1 \mathrm{GeV})$ energy deposit in tbis calorimeter.

The second objective is to detect and identify all final state products, for which we used the downstream detectors. We identify the charge of the final state particles from the upstrearn and downstream scintillator hodescopes and energy from proton and neutron region calorimeters. Figure $3,(a)$ and (b) shows the measured charge distribution for heavy-ion fragments and the measured energy distribution for protons. We have achieved about $2.7 \%$ resolution in charge measurement and $0.6 / \sqrt{E}$ for energy measurement, both of which are adequate to identify low multiplicity peripheral channels (1p, in or $1 \mathrm{p} 1 \mathrm{n})$. The resolution in the charge measurement is good enough to separate all charges from 
$Z=1$ to $Z=14$.
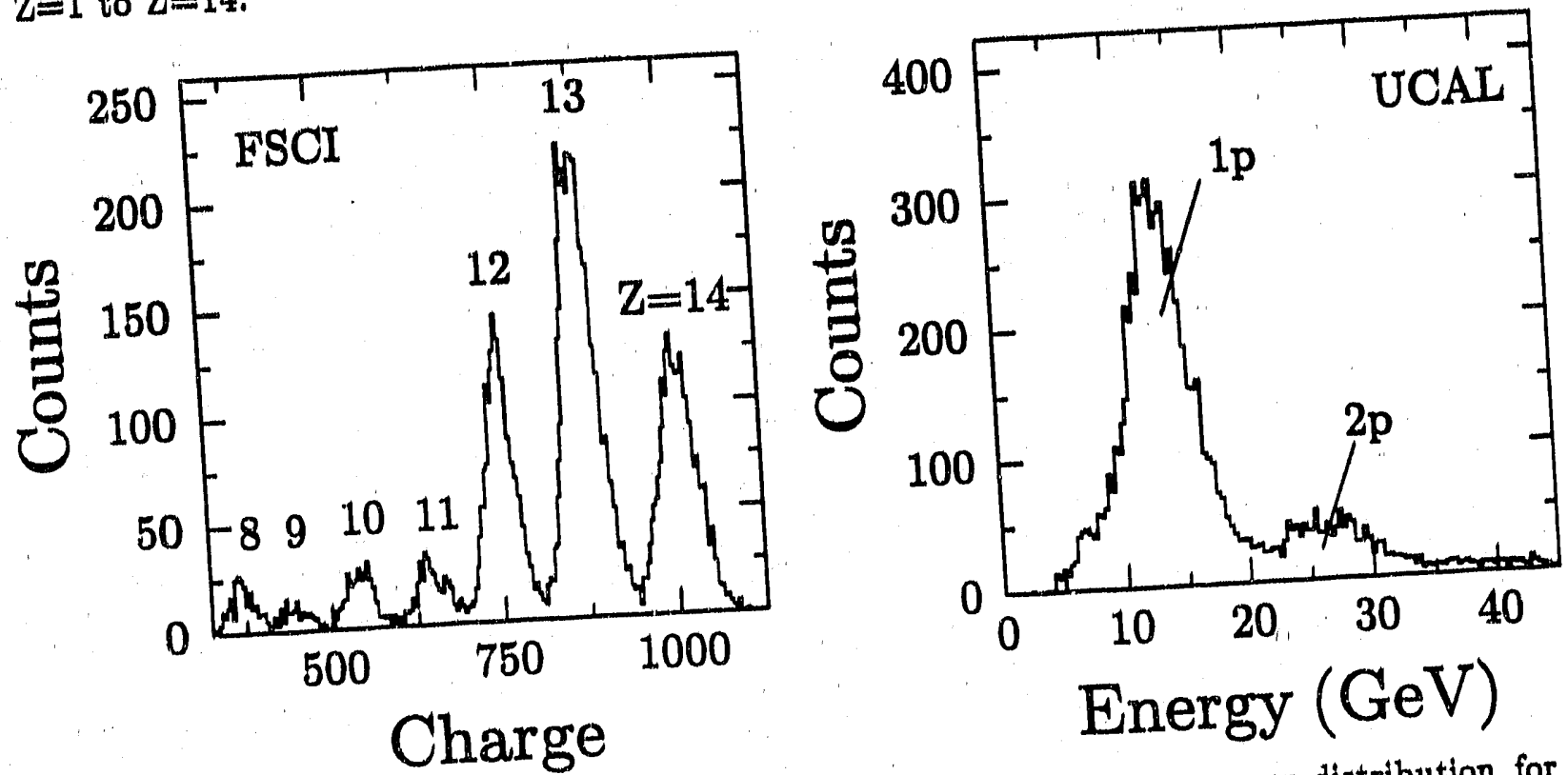

Figure 3: (a) Charge distribution for heavy lons and (b) calorimeter energy distribution for protons in peripheral data.

With these tools, we analyze raw data tapes and extract candidates for nuclear and peripheral events for further analysis and record them in a secondary set of $8 \mathrm{~mm}$ tapes. This procedure reduced our data sample by a factor of 4 , mostly due to the rejection of events containing downstream interactions. These tapes were used at this stage to study cross sections for channels with low-multiplicity final states, such as $1 \mathrm{p}+{ }^{27} \mathrm{Al}, 1 \mathrm{n}+{ }^{28} \mathrm{Si}$, and $1 p+1 n+{ }^{26} \mathrm{Al}$. The more complex peripheral channels are contaminated and information from the tracking chambers is required to reduce the background.

The E814 tracking chambers (described in detail elsewhere $[8,9]$ ) have been used previously to study events produced via central interactions, in which only minimum ionizing particles are present. One of the problem we faced was to utilize these chambers in peripheral interactions, in which a highly charged ion is present in the final state. The heavy-fragment tends to produce more delta rays and other noise problems in the chambers making it difficult to obtain a good tracking resolution with the tracking software developed for minimum ionizing particles. 
peripheral tivents after using our event reconstruction. With this information we are able to easily identify multinucleon emission channels. As an example, we show in Figure 4 (b) the magnetic rigidity for the events with measured $\mathrm{Z}=13$. Different peaks correspond to different isotopes and we can clearly identify $1 \mathrm{p}, 1 \mathrm{p} 1 \mathrm{n}$ and $1 \mathrm{p} 2 \mathrm{n}$ events.
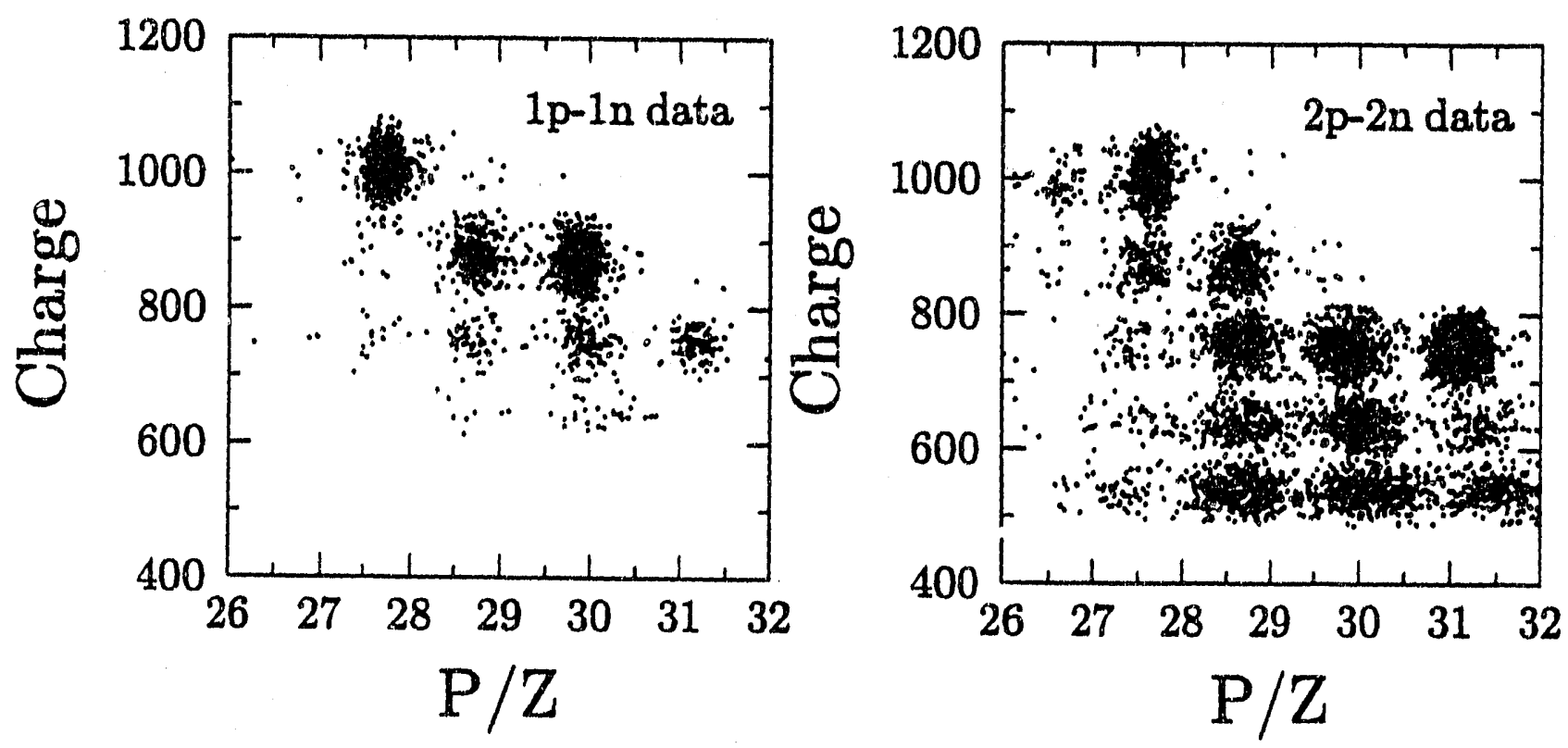

Figure 5: Two dimensional plots of the heavy ion charge (on a scale where $\mathrm{Z}=14$ corresponds to 1000 ) vs its magnetic rigidity (P/Z). Each "island" corresponds to a different final state. (a) $1 p$ triggers; (b) $2 p$ triggers.

In Figure 5, (a) and (b) we show the measured charge against the magnetic rigidity of the heavy-ion for a collection of events extracted through $1 \mathrm{p}-1 \mathrm{n}$ tapes and $2 \mathrm{p}-2 \mathrm{n}$ tapes. (Figure 4 are two projections of this plot.) We can identify a large number of peripheral channels from these and we have a good possibility of extracting rare channels such as $3 p+{ }^{25} \mathrm{Na}, 3 p+1 n+{ }^{24} \mathrm{Na}, 2 \mathrm{p}+1 \mathrm{~d}+{ }^{24} \mathrm{Na}, 4 \mathrm{p}+2 \mathrm{n}+{ }^{22} \mathrm{Ne}, 2 \mathrm{p}+1 \alpha+{ }^{22} \mathrm{Ne}$ etc. We are currently investigating the possibilities of extraction of some of these channels for full event reconstruction. 


\subsection{Physics Analysis}

One of the main goals of the E814 peripheral program was to measure the excitation energy of the ${ }^{28} \mathrm{Si}$ for possible channels and to get an insight into the Giant Dipole resonance. This can shed light into the Weiszäcker-Williams (WW) method and also to the possible multiphoton excitations. The E814 apparatus allows us to fully reconstruct peripheral channels with 2 kinematic constraints in order to study in detail such effects.

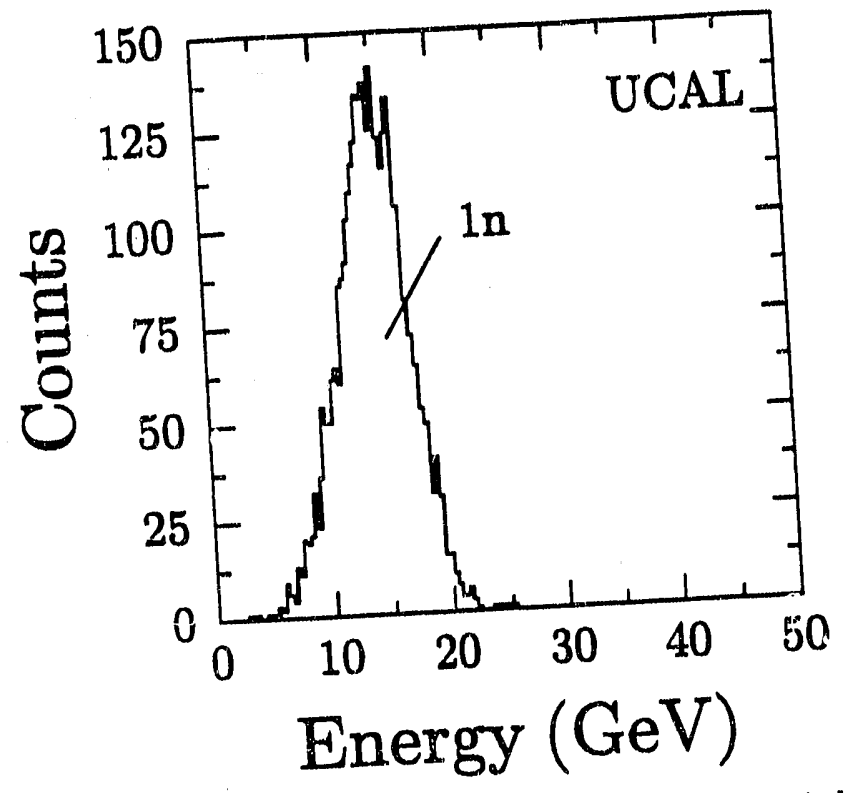

Figure 6: Neutron calorimeter energy distribution for peripheral events.

We began this work with the simplest decay channel $1 \mathrm{p}+{ }^{27} \mathrm{Al}$. This channel, apart from physics understanding, provides an excellent tool to understand the systematic problems associated with the detectors and resolution measurements. We also developed a simuiation program which simulates the $1 p$ decay. It takes the known low energy photo nuclear cross section data for the ${ }^{28} \mathrm{Si}(\gamma, \mathrm{p})^{27} \mathrm{Al}$ channel and convolutes it with the WW photon spectrum and gives an excellent representation for our data. The preliminary result of this $1 p$ analysis $w$ is presented at the April 1991 APS meeting. The work for $1 \mathrm{p}$ is now completed, and the results were submitted for publication in Physical Review C. A copy of this paper is included in this report as Appendix A. 
We are now working on a somewhat more difficult channel, $1 n+{ }^{27} \mathrm{Si}$. This channel is interesting for many reasons. In literature, very limited data is available for low energy measurements because of the difficulty in measuring and detecting the neutron. This channel can also provide an understanding of how well calorimetry works in the reconstruction of exclusive events containing final state neutrons, and could lay the groundwork for studies of events with final state multineutron clusters like ${ }^{2} n$ or ${ }^{4} n$.
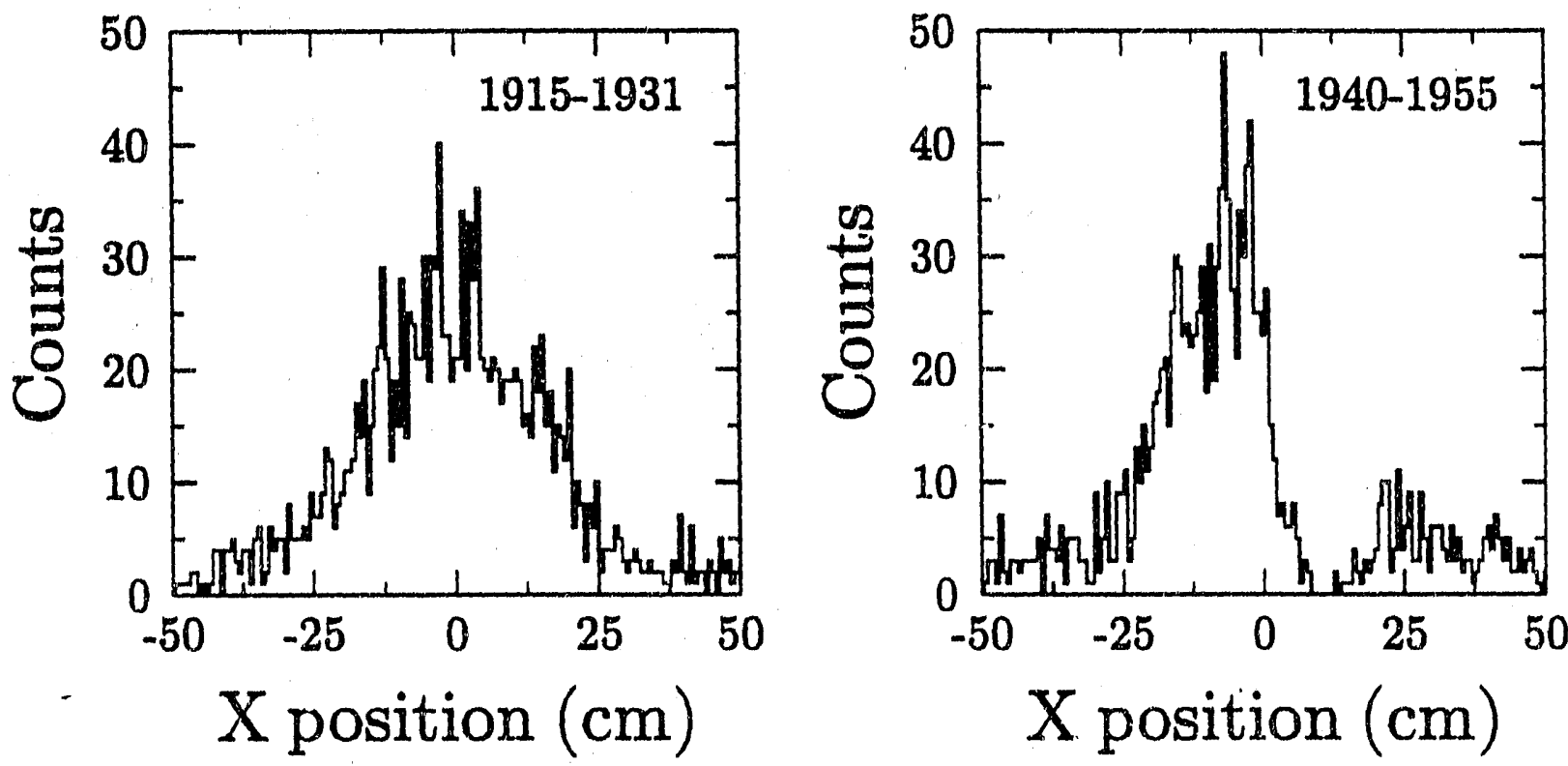

Figure 7: Horizontal position distribution for 1n data. (a) Portion of data sample with full calorimeter trigger; (b) portion of sample with one calorimeter module missing from trigger.

In Figure 6, we show the measured energy in the neutron calorimeters for the $1 n$ events. The efficiency of the neutron calorimeters are about $96 \%$. The low energy tail corresponds to neutrons with late showers which deposit very little energy in the calorimeter. In Figure, 7 (a) and (b) we show the measured $x$ positions at these calorimeters for the early part of the run and later part of the run. Due to a bad cable from one of the calorimeters (between $0.21 \mathrm{~cm}$ ) we have lost the contribution of one of the calorimeter modules to the trigger sum during the second part of the run which results in a gap in the position distributions. Fortunately, about half of the data does not suffer from this problem. Our calorimeter algorithms provide about $\approx 2 \mathrm{~cm}$ resolution in $\mathrm{x}$ and a little worse resolution 
in the $\mathrm{y}$ direction.

Figure 8 shows the measured cross sections for 1n data (white circles) together with $1 \mathrm{p} 1 \mathrm{n}$ (black circles). The fit is a two component fit described in the 1p paper. Both of these show roughly a $Z^{1.8}$ behavior with the target charge for the EMD part although the fit is s: Jwn only on 1n data. 1pln data suffered due to low statistics. The total cross section shows a non-negligible contribution from the "soft" nuclear part, specially for low $\mathrm{Z}$ targets.

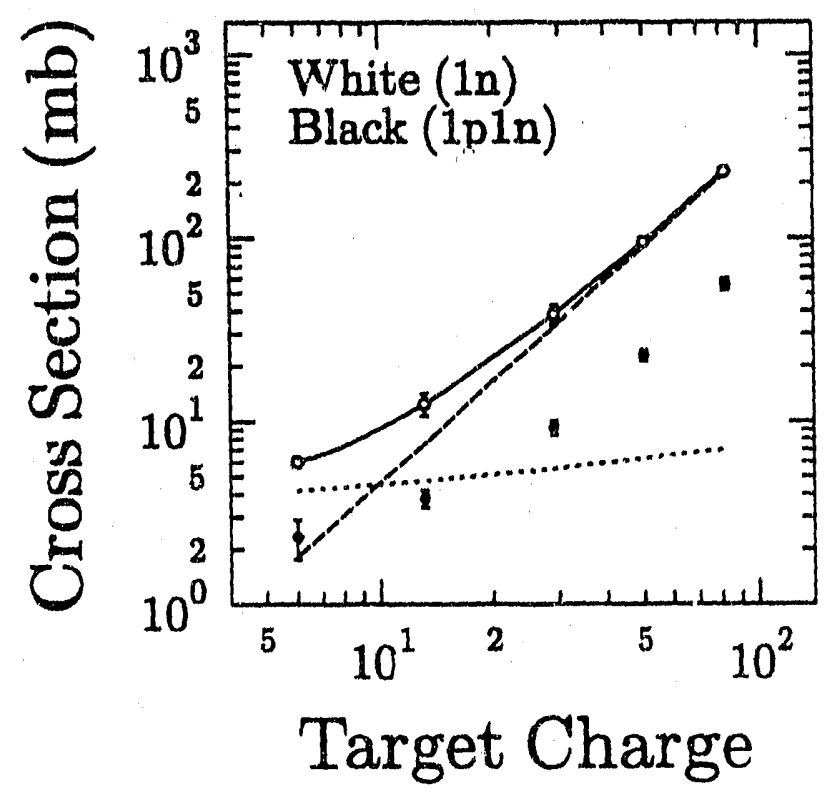

Figure 8: Cross section vs, target charge for 1 n (white circles) and 1n1p (black circles) data. The lines show the electromagnetic (dashed), nuclaar (dotted), and total (solid) composition for the 1n data.

We carry out the same kinematic reconstruction process for the In data sample as was used for the $1 \mathrm{p}$ sample, although we expect considerably worse resolution due to the lack of magnetic tracking for the neutron. This is easily seen in Figure 9, where we show the reconstructed momentum spectra for the $1 \mathrm{p}$ and $1 \mathrm{n}$ events. Note that the energy distribution in the in case is not quite symmetric, which we believe is a property of the calorimeter, arising from cases in which the neutron shower starts deep in the calorimeter. One of the challenges in this analysis, which is still in progress, will be to correctly handle 
the calorimeter response in the kinematic reconstruction. One of the tools at our disposal is the reconstructed angular distribution of the polar decay angle in the center-of-mass frame $\left(\theta^{*}\right)$.
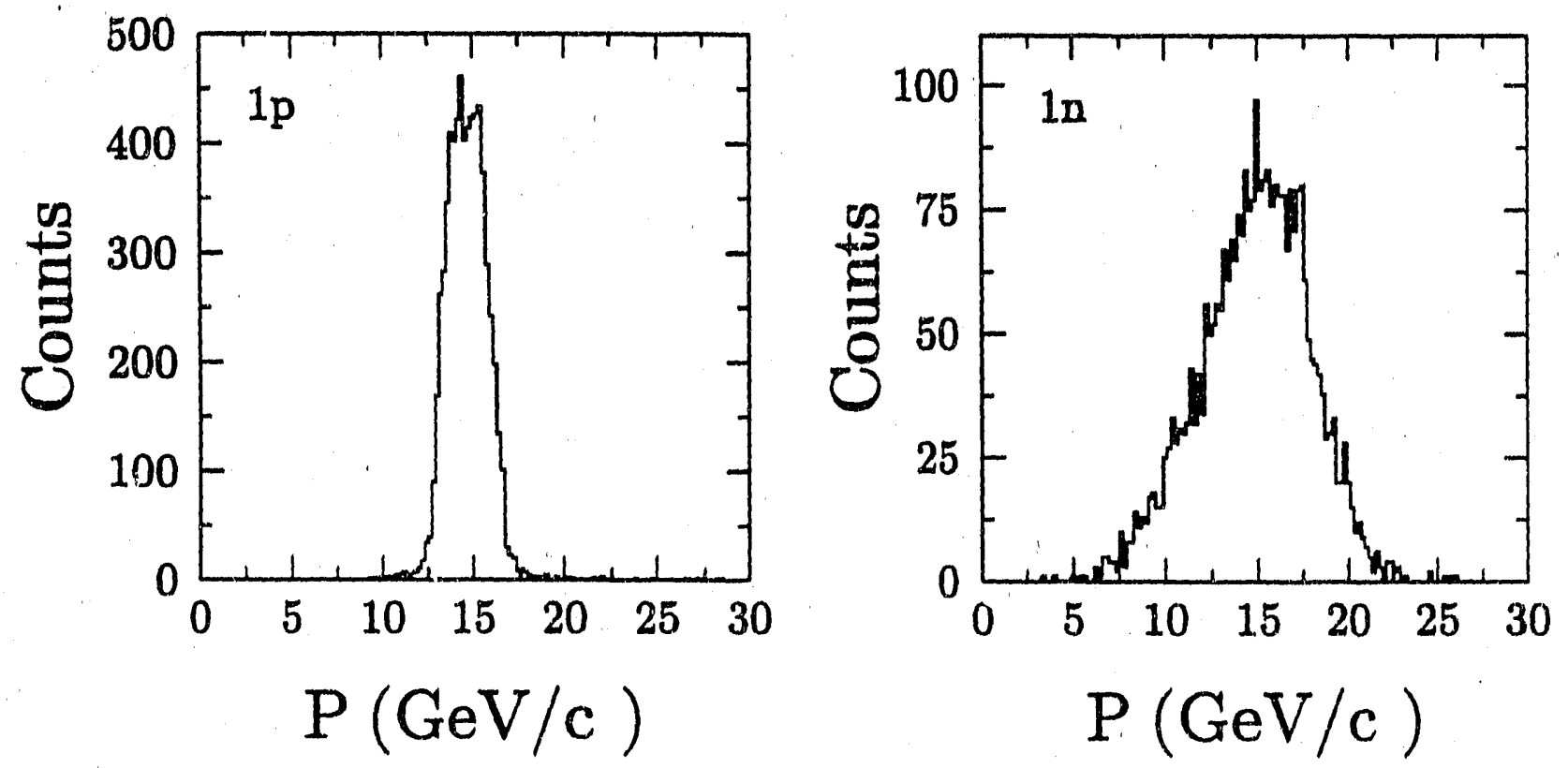

Figure 9: Momentum distributions from kinematic fit for (a) protons in $1 p$ events and (b) neutrons in $1 \mathrm{n}$ events. The resolution for the $1 \mathrm{n}$ data is considerably worse than that for the $1 \mathrm{p}$ events due to the use of the calorimeter rather than magnetic tracking for energy determination.

In Figure 10a we show the angular distribution found for the $1 \mathrm{p}$ events, which is consistent with isotropy, and in Figure $10 \mathrm{~b}$ we show the corresponding quantity for the $1 \mathrm{n}$ events, which is clearly very different. The severe anisotropy in this case arises, we believe, from an improperly weighted measurement of the neutron energy, which, in the current analysis is assumed to be gaussian in nature. (Too low or too high energy measurements in UCAL tends to put events forward or backward in the CM system, artificially giving this shape.) We plan to develop a liklihood technique which will permit the introduction of a nongaussian response function for the calorimeter, and monitor the effect on the angular distribution, in which we will assume the same isotropy as has been measured in the $1 \mathrm{p}$ case.

Figure 11, (a) and (b) compare the excitation energy distribution for $1 \mathrm{p}$ and $1 \mathrm{n}$. The 

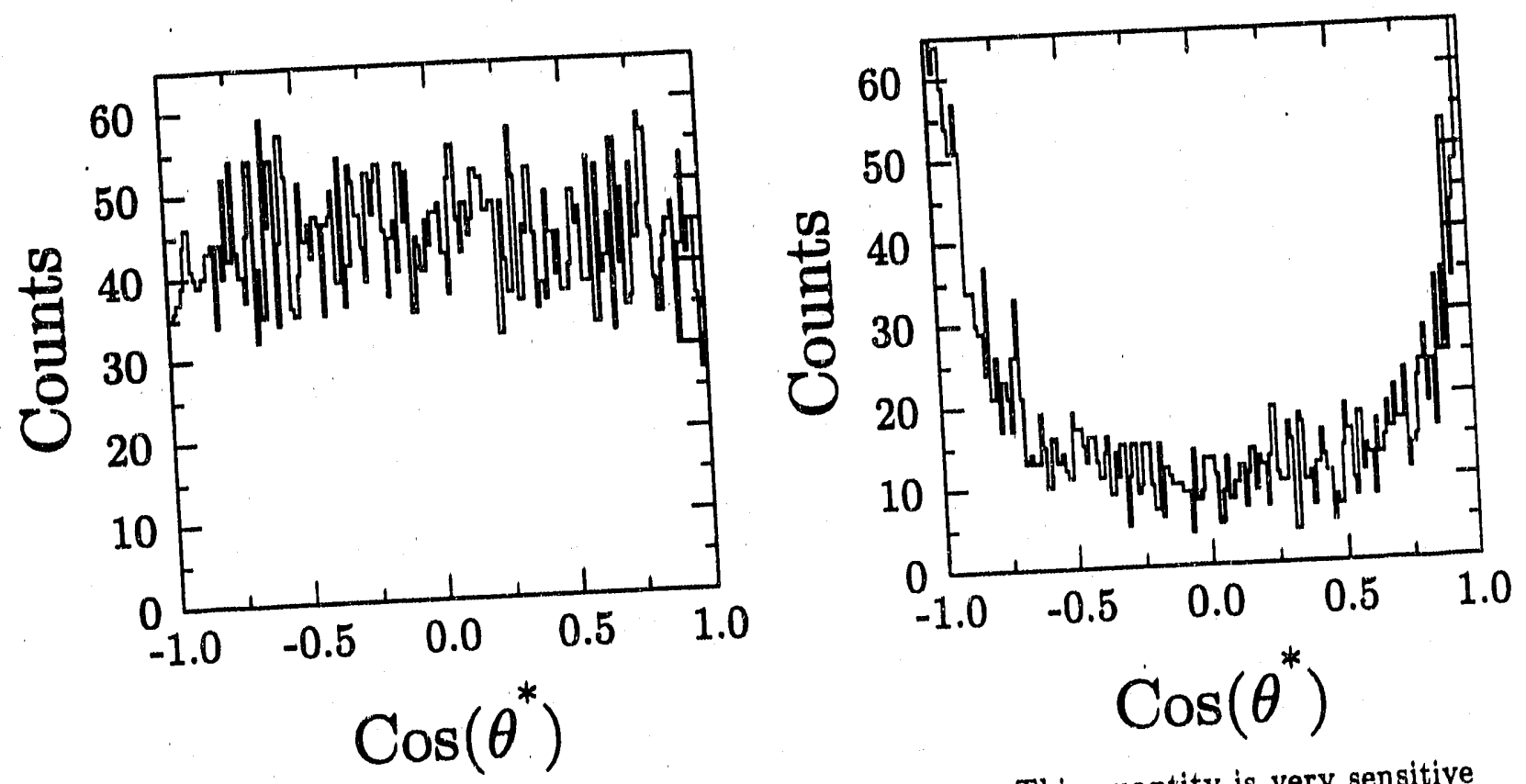

Figure 10: Distribution in $\cos \left(\theta^{*}\right)$ for (a) $1 \mathrm{p}$ and (b) in events. This quantity is very sensitive to errors in the energy determination. The lack of uniformity in the in case probably arises from the non-gaussian behaviour of the calorimeter response.

kinematic thresholds for these events are $11.59 \mathrm{MeV}$ and $17.18 \mathrm{MeV}$ respectively. Clearly the neutron energy and position measurement also affects the excitation energy for the event. We plan to present preliminary results for this channel at the April 1992 APS meeting.

2.4 Studies of $2 \mathrm{p}$ emission in the EMD of ${ }^{28} \mathrm{Si}$ and reconstruction of the invariant mass for the $2 \mathrm{p} 0 \mathrm{n}$ decay channel

Following the encouraging results obtained in the reconstruction of the invariant masses in the dissociation of ${ }^{28} \mathrm{Si}$ by emission of one proton, we are also pursuing a study of EMD into the $2 p$ emission channels, particularly the $2 p 0 n$ decay channel. These decay modes require larger photon energies to be produced, and might reveal new aspects in the excitation process of ${ }^{28} \mathrm{Si}$. Also, the event reconstruction of the two nucleon emission channel, $2 \mathrm{p} 0 \mathrm{n}$, opens up the possibility for looking for correlated $2 \mathrm{p}$ emission if this can happen in peripheral collisions in the energy region spanned by our data. 

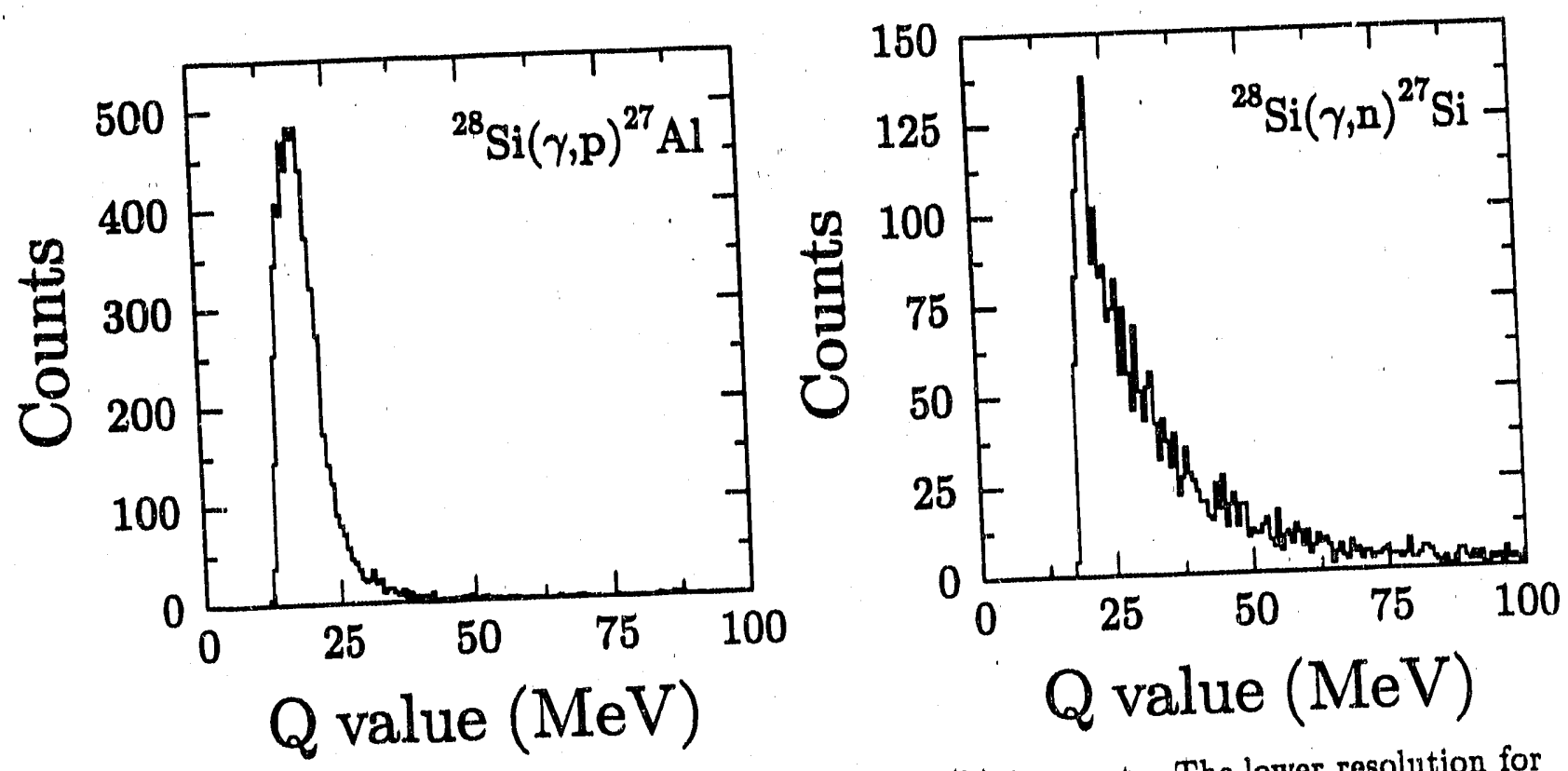

Figure 11: Excitation energy distribution for (a) $1 \mathrm{p}$ and (b) in events. The lower resolution for the neutron position and energy causes a lower resolution in the excitation energy determination.

\subsubsection{Event Selection}

To select good "EM events" (events from the EMD of ${ }^{28} \mathrm{Si}$ ) we use the same method described in detail in refs. [3] and [6]. To select good 2pxn events we' impose cuts on the charge and energy of the heavy ion $(\mathrm{Mg})$ as well as of the protons, measured in the corresponding forward scintillators and calorimeters (details of the analysis procedure are described in ref. [6]), and we also demand appropriate energy in the neutron calorimeters. To further improve the quality of the selection procedure, we require the magnetic rigidity $\mathrm{P} / \mathrm{Z}$ of the heavy ion (see Figure 4) to be within certain limits. This step is needed since inefficiency and poor energy resolution in the neutron calorimeters can allow background (primarily $2 p(x+1) n$ ) events to be present in the $2 p x n$ sample. Combined with the charge information, this analysis permits us to identify the mass number of the heavy ion, thus enabling the extraction of a cleaner sample of $2 p x n$ events.

To perform the heavy ion $\mathrm{P} / \mathrm{Z}$ analysis we use information provided by the drift chambers of the E814 forward spectrometer, and we reconstruct the heavy ion track. 


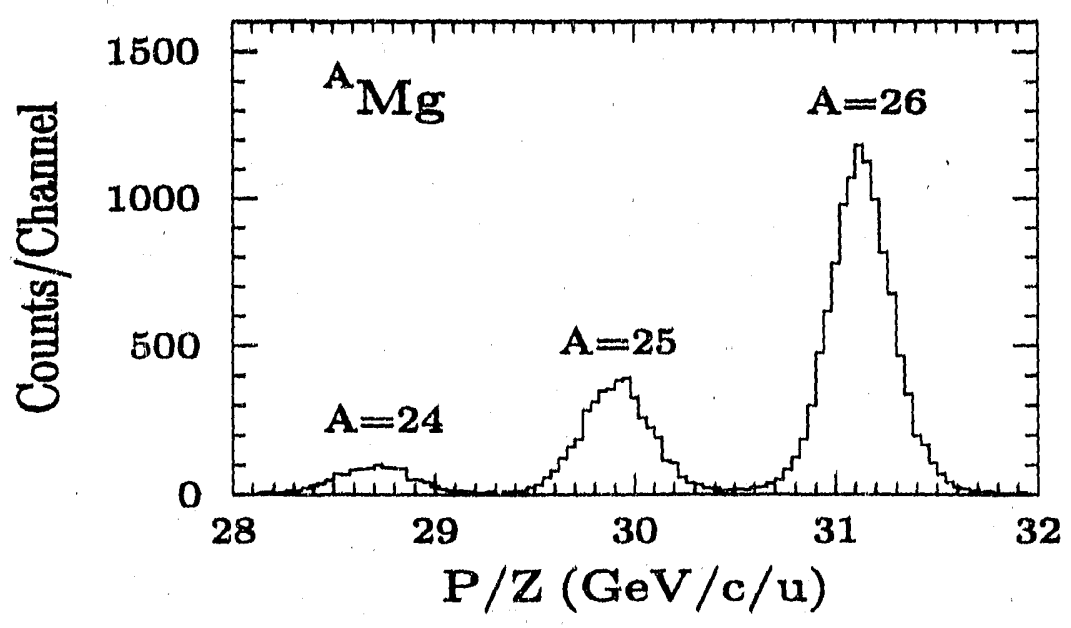

Figure 12: Momentum distribution of $\mathrm{Mg}$ isotopes corresponding to 2pxn emission channels from the decay of ${ }^{28} \mathrm{Si}^{*}$.

Figure 12 shows the $\mathrm{P} / \mathrm{Z}$ distribution for events where the heavy ion has charge number $\mathrm{Z}=12$. Indicated in the figure are the mass numbers corresponding to the various $\mathrm{Mg}$ isotopes, and we note the clean separation between the different groups.

This mathod of mass identification of the heavy ion is currently being applied to many other studies of the E814 peripheral collision data, such as studies of fragmentation processes, EMD into multi-nucleon emission channels and search for rarer decay modes, involving for example the emission of $\alpha$ particles. It is also used in the selection of specific samples of events for studies involving invariant mass reconstruction, which are presently being done for the $2 \mathrm{p} 0 \mathrm{n}$ and $1 \mathrm{n}$ decay channels.

\subsubsection{Cross Sections Studies}

Preliminary cross sections for the EMD of ${ }^{28} \mathrm{Si}$ into $2 \mathrm{p} 0 \mathrm{n}, 2 \mathrm{p} 1 \mathrm{n}$ and $2 \mathrm{p} 2 \mathrm{n}$ decay channels were extracted for targets of $\mathrm{Pb}, \mathrm{Sn}, \mathrm{Cu}$ and $\mathrm{Al}$. Figure 13 shows the cross sections as a function of the target $Z$. Under the assumption that the Coulomb and the nuclear parts do not interfere, and that the nuclear component scales as the sum of the target 


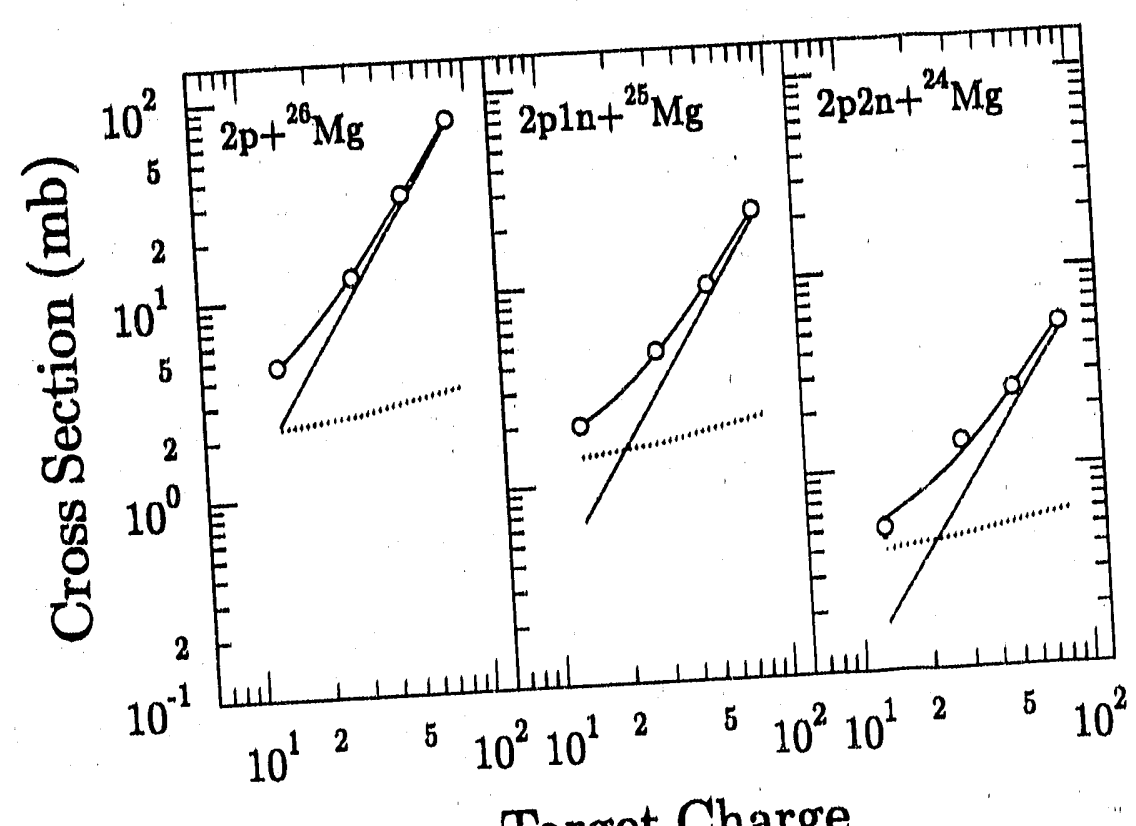

Figure 13: Cross sections for the $2 \mathrm{p} 0 \mathrm{n}, 2 \mathrm{p} 1 \mathrm{n}$ and $2 \mathrm{p} 2 \mathrm{n}$ decay channels from ${ }^{28} \mathrm{Si}^{*}$ as a function of the target $Z$.

and projectile perimeters, the electromagnetic component scales approximately as $Z^{1.8}$, in agreement with other EMD studies [11]. In the figure, the dashed curve represents the EM component, the dotted curve is for the nuclear component, and the solid curve is for the sum of the two components.

\subsubsection{Invariant mass reconstruction studies for the $2 \mathrm{p} 0 \mathrm{n}$ decay channel}

To handle the kinematic reconstruction of the 3-body decay channel, we found it convenient to consider it as a composite 2-body decay system. We emphasize that this is not a physical assumption, and that we can always convert the quantities extracted in the frame of any 2-body decay frame to the original frame of the decaying projectile by doing the appropriate Lorentz transformations.

We consider the following decay scheme: 


$$
\begin{aligned}
& \text { - }{ }^{28} \mathrm{Si} \rightarrow{ }^{26} \mathrm{Mg}+\mathcal{D} \text { ("diproton") } \\
& \text { - } \mathcal{D} \rightarrow \mathrm{p}+\mathrm{p}
\end{aligned}
$$

\section{We define the following quantities:}

- $\mathrm{Ex}_{1}$ is the excitation energy of the excited ${ }^{28} \mathrm{Si}^{*}$.

- $Q_{2}$ is the mass of the diproton less the rest masses of the two protons.

- $\theta^{*}$ is the polar angle of the ${ }^{26} \mathrm{Mg}$ momentum vector with respect to the beam momentum vector in the rest frame of the ${ }^{28} \mathrm{Si}$ projectile.

- $\phi^{\prime \prime}$ is the azimuthal angle of the decay plane about the beam momentum vector, in the first 2-body decay subsystem, referred to the horizontal plane.

- $\theta_{p}$ is the polar angle of the momentum vector of the proton going forward with respect to the momentum vector of the diproton in the rest frame of the diproton.

- $\phi_{p}$ is the azimuthal angle of the decay plane about the diproton momentum vector, in the diproton frame, referred to the decay plane of the first 2-body decay subsystem.

The basic steps for event reconstruction are described in detail in ref. [3]. However a number of extra procedures are introduced to handle the reconstruction of the two proton tracks, since they lie close together in a considerable fraction of the events. The reconstructed mass of the diproton shows a distribution concentrated at very low values, as is shown below, and under these circumstances the momentum vectors of the two protons are almost identical, so they follow practically the same trajectory.

The main new features introduced in the reconstruction program are:

- A better definition of the "roads" defined by the pads in DC2 and DC3 to take into account regions of different pad widths in each chamber. These "roads" are used to perform an initial selection of the TDC hits belonging to each track. 
- Inclusion of a second alternative for creating the "roads" corresponding to each track by swapping, in one of the chambers, the pad information initially assigned to each track.

- For the cases where the two proton tracks cross the same pad in one of the chambers, inclusion of a second alternative for fitting the track through the TDC hits by swapping, in the chamber having the common pad, the set of TDC hits initially associated to each proton track. Studies done with simulated events showed that improper assignment of the set of TDC hits to each track was not unlikely to occur, resulting in wrong values for the reconstructed quartities.

- Inclusion of special criteria to choose the cocrect alternative for the proton hits, which consists of reconstructing the tracks for both configurations, going through the magnets up to the target, and making the choice based on (a) how mainy hits have been found in DC1, (b) how many TDr hits are found to lie on each track, and (c) ihe combined $\chi^{2}$ values of the fit to the two tracks. Tests done with simulated events show that the method works well.

By using oimulated events we also determine the resolution attainable in the reconstruction of invariant masses and decay angles. Basically, we generate the positions of the tracks in all the detection planes of our apparatus, in which we itclude distortions due to multiple scattering in the target and in the materials present in the path of the particles, and resolution of the detectors. The set of events is then reconstructed and we compare the results from the reconstruction to the quantities used to produce the event. Figure 14 shows the differences between these two quantities for: $(a)$ the excitation energy of the ${ }^{28} \mathrm{Si}^{*} ;(b)$ the mass of the diproton; $(c)$ the polar angle $\theta^{*} ;(d)$ the azimuthal angle $\phi^{*} ;$ (e) the polar angle $\theta_{p i}(f)$ the azimuthal angle $\phi_{p}$. The distributions shown correspond to the $\mathrm{Pb}$ target. Our resolution is about $1.7 \mathrm{MeV}$ in the excitation energy of the ${ }^{28} \mathrm{Si}^{*}$ and $0.5 \mathrm{MeV}$ in the mass of the diproton, which are remarkable when we consider that the beam energy is of hundreds of $\mathrm{GeV}$. The angular distributions also show good overall 

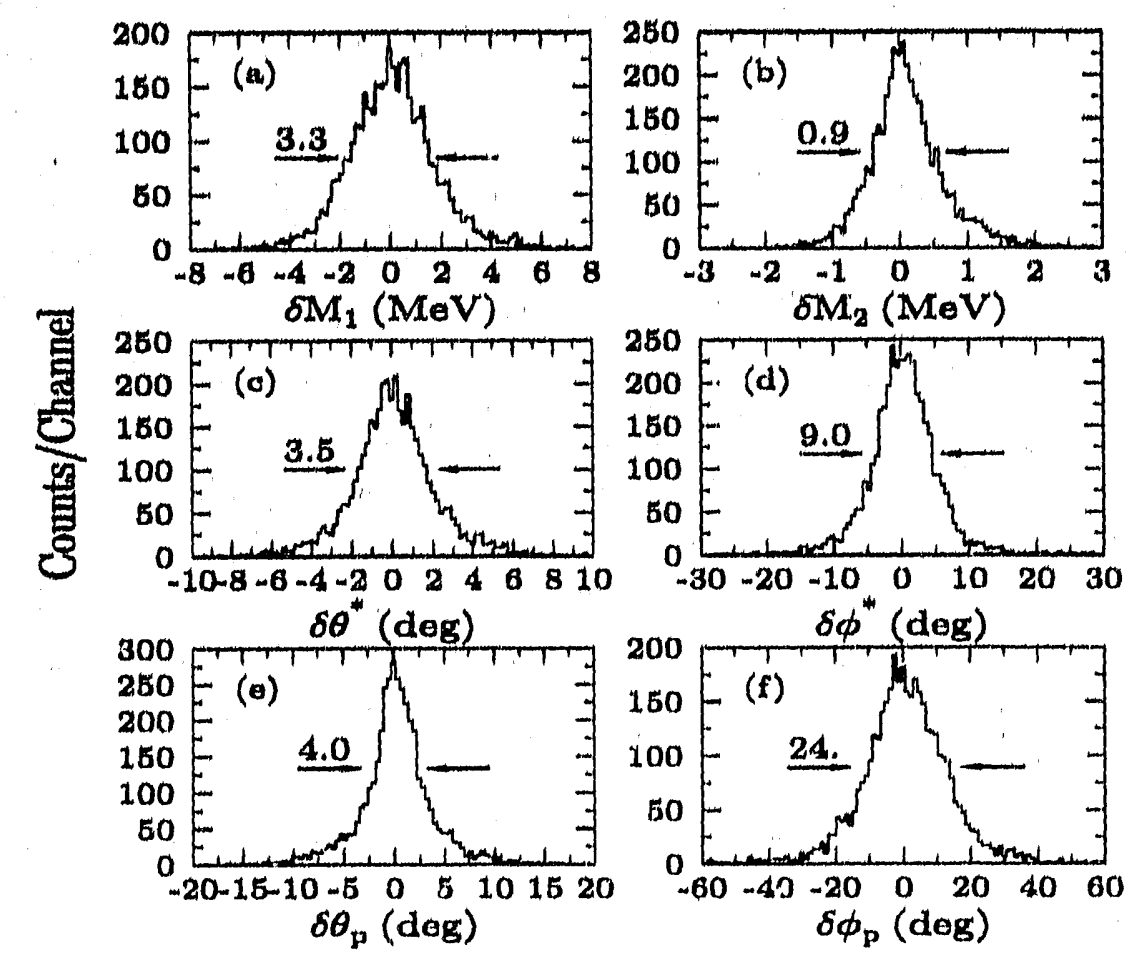

Figure 14: Distribution of the differences between reconstructed quantities and the ones used to produce the event.

resolution except for the azimuthal angle of the diproton decay, which is understandable if we remember that most of the time the two protons are quite close together.

Some preliminary reconstructed data are presented in Figs. 15 and 16. Figure 15 shows the distributions of $(a)$ the excitation energy of the ${ }^{28} \mathrm{Si}^{*}$ and $(b)$ the mass of the diproton system (less the rest masses). In Figu e 16 we show the angular distributions of the decay. The sharp rise above the threshold that we see in Figure 15(a) gives an indication of our good resolution in excitation energy. If we compare this distribution with the one for the excitation energy of ${ }^{28} \mathrm{Si}^{*}$ in the $1 \mathrm{p}$ decay channel $\mathrm{n}$ the $1 \mathrm{p}$ decay channel (Figure $\varepsilon$ of ref. [3]), we note that in the present case of $2 p$ emission the high energy tail extends further out. In part (b) of Figure 15 we see that the diproton mass distribution is peaked at very low values, which means that the two protons will have close trajectories, as noted 


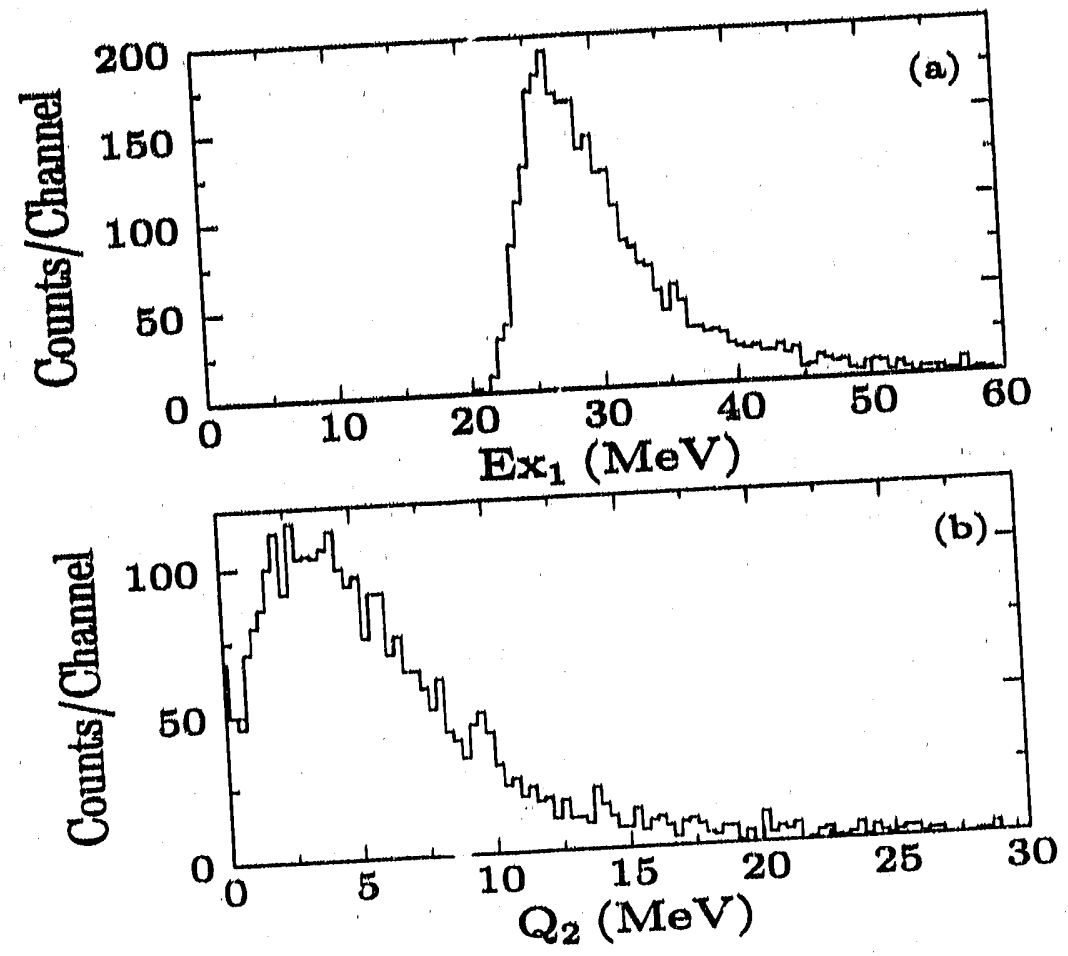

Figure 15: Experimental Distribution of $(a)$ excitation energy of ${ }^{28} \mathrm{Si}^{*} ;(b)$ mass of the diproton system (less the rest masses).

above.

The $\cos \left(\theta^{*}\right)$ distribution shown in Figure $16(a)$ indicates that apparently the ${ }^{26} \mathrm{Mg}$ is emitted preferentially in the forward direction. However studies made using simulated events show that this behaviour could be due to misalignment of the detectors or imprecise information about the incident beam momentum. Studies of the incident beam momentum provided by the Gauss Clock (a pulse train provided by the AGS whose frequency is related to the instantaneous magnetic rigidity of the extracted beam) indicate that from one set of runs to another the beam rigidity $\mathrm{P} / \mathrm{Z}$ can vary by as much as 0.3 $\mathrm{GeV} / \mathrm{c} / \mathrm{u}$. On the other hand our studies based on simulation indicate that a systematic error of 0.2 in the beam $\mathrm{P} / \mathrm{Z}$ value already produce angular distributions with the $\cos \left(\theta^{*}\right)$ peaked at one of the extremes. This effect is caused by an error in the Lorentz factor, and 
$E_{T}$ distributions measured in the participant calorimeter.

The charged multiplicity work was the $\mathrm{Ph} . \mathrm{D}$. thesis of one of our graduates students, $\mathrm{M}$. $K$. Jayananda, and this research was completed during the past year. A copy of his thesis is included as Appendix B of this report. The topics covered included total multiplicity distributions for the three targets used in the central data-taking ( $\mathrm{Al}, \mathrm{Cu}$, and $\mathrm{Pb}$ ), a study of $d N / d \eta$, including an evaluation of the negative binomial parameters, and a study of two-particle correlation functions. The techniques and conclusions are reported in detail in Appendix B. A paper on the charged multiplicity distributions measured in E814 has been submitted to Physical Review C [7].

The analysis of the $E_{T}$ distributions from the participant calorimeter is still underway. It is discussed in detail in the following section.

\subsection{Studies of $E_{T}$ with the Participant Calorimeter}

\subsubsection{Detector Description}

Table 2: List of the mechanical properties of the participant calorimeter

\begin{tabular}{|c|c|c|c|c|}
\hline & EM1 & EM2 & HAD1 & HAD2 \\
\hline $\begin{array}{c}\text { Number of } \mathrm{Pb} \text { plates } \\
\text { Thicknes of Pb plates }\end{array}$ & $\begin{array}{c}5 \\
1.0 \mathrm{~cm}\end{array}$ & $\begin{array}{c}5 \\
1.0 \mathrm{~cm}\end{array}$ & $\begin{array}{c}20 \\
1.0 \mathrm{~cm}\end{array}$ & $\begin{array}{c}19 \\
1.0 \mathrm{~cm}\end{array}$ \\
\hline Number of Fe plates & 1 & 1 & 1 & 1 \\
Thicknes of Fe plates & $1.6 \mathrm{~cm}$ & $1.6 \mathrm{~cm}$ & $1.6 \mathrm{~cm}$ & $1.6 \mathrm{~cm}$ \\
\hline Number of scint. layers & 6 & 6 & 24 & 23 \\
Number of scint. plates & 768 & 768 & 3072 & 3072 \\
Thicknes of scint. plates & $0.3 \mathrm{~cm}$ & $0.3 \mathrm{~cm}$ & $0.3 \mathrm{~cm}$ & $0.3 \mathrm{~cm}$ \\
Type of scint. plates & $\mathrm{BC} 408$ & $\mathrm{BC} 408$ & KSTI-430 & KSTI-430 \\
\hline Number of PMT's & 128 & 128 & 128 & 128 \\
\hline
\end{tabular}

The participant calorimeter is designed to be approximately azimuthally symmetric, 

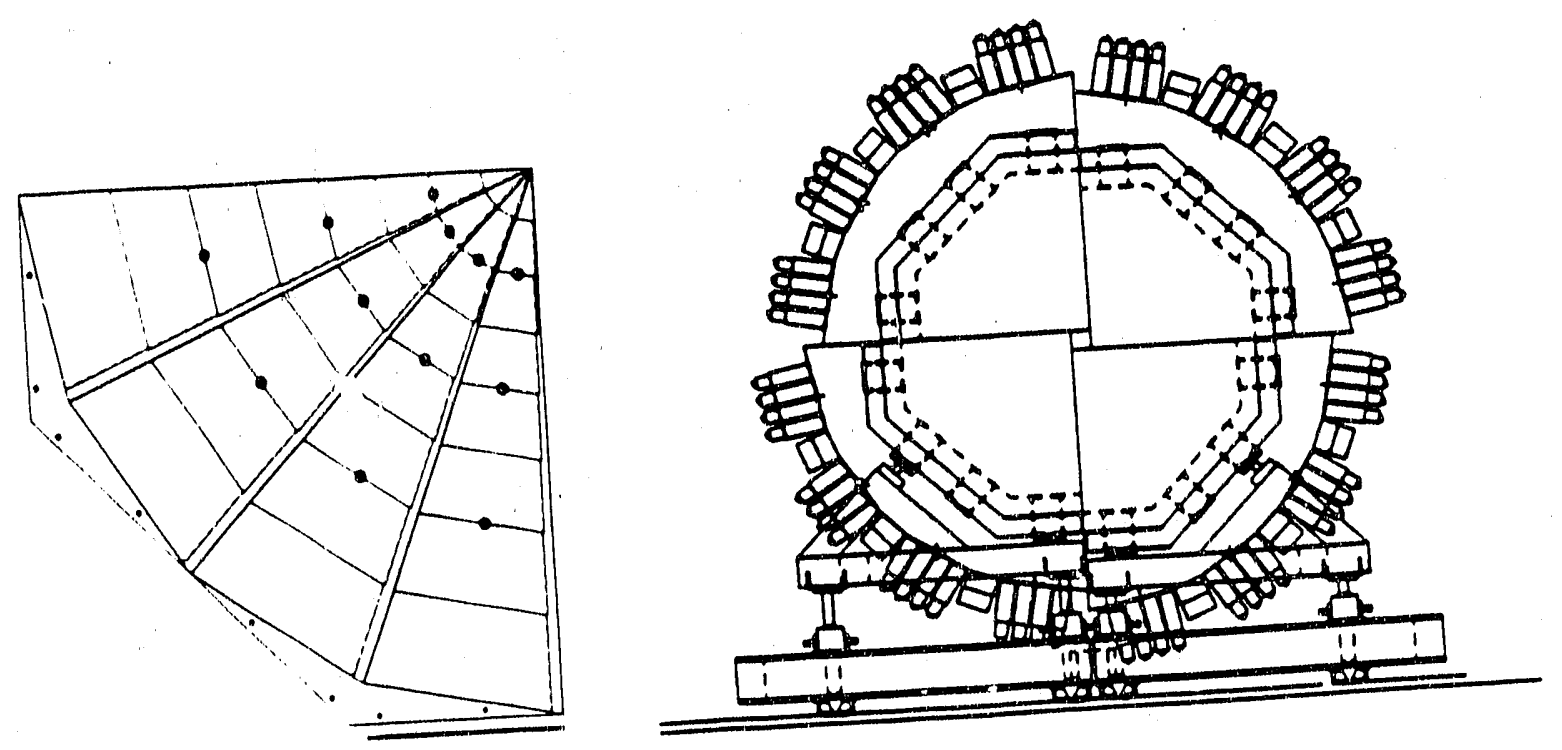

Figure 17: Participant calorimeter, seen by the beam. The left drawing details transverse segmentation in quatrant IV.

coveririg the polar angle $1.6^{\circ}<\theta<48.0^{\circ}$, corresponding to pseudorapitity region 0.81 $<\eta<4.3$. It uses lead plates as radiators and plastic scintillators coupled with optical fibers for sampling readouts. Every 6th lead plate is substituted with an iron plate for mechanical stability.

The calorimeter has a depth of four interaction lengths and a radius of 33 inches. It is segmented tranversely into 8 radial and 16 azimuthal segments and longitudinally into 4 depth segments, and is read out with an array of 512 phototubes. A list of the mechanical properties is given in Table 2 and a drawing of the calorimeter is shown in Figure 17.

\subsubsection{SEB Test beam for Calibration}

The procedure to calibrate PCAL is that a group of towers accessible to the beam which lie in the horizontal azimuthal segment (pie) with different sizes and positioned at different values of pseudorapidity can be calibrated first with SEB beam, with the reasonable 
assumption that all towers at the same pseudorapidity receive the same amount of $E_{T}$ the calibration is then spread around the detector according to the calibrated tower with similar pseudorapidity and same depth.

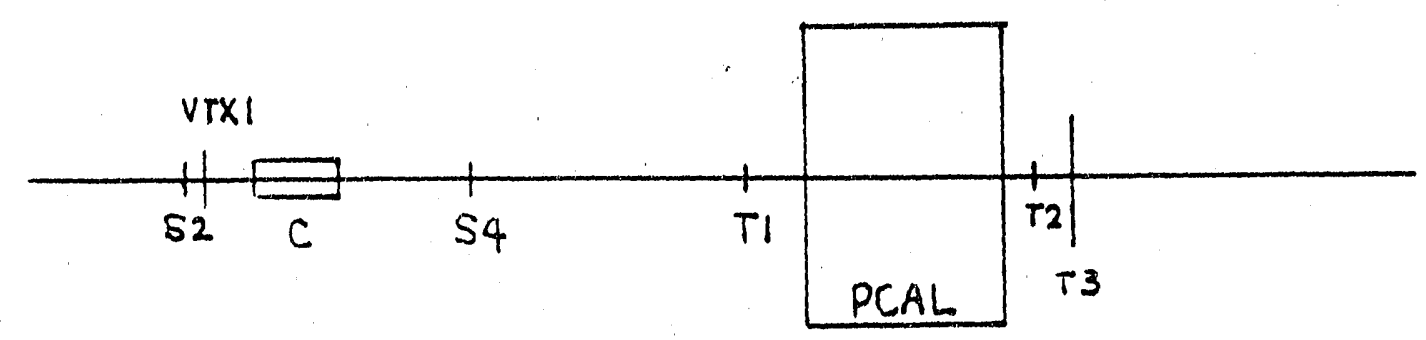

Figure 18: Setup used in the calibration of the participant calorimeter.

Table 3: Conditions for the calibration runs, showing the techniques employed for the particle identification

\begin{tabular}{|c|c|c|c|c|c|}
\hline momentum(Gev/c) & $\mu$ & $\mathrm{e}$ & $\pi$ & $\mathrm{p}$ & gas in Cherekov \\
\hline 1.0 & $\mathrm{~T} 1, \mathrm{~T} 2$ & $\mathrm{C}$ & no C & TOF & air(1.0atm) \\
2.0 & $\mathrm{~T} 1, \mathrm{~T} 2$ & $\mathrm{C}$ & no C & TOF (limit) & air(1.0atm) \\
-4.0 & $\mathrm{~T} 1, \mathrm{~T} 2$ & $\mathrm{C}$ & no C & - & air(1.0atm) \\
+4.0 & T1,T2 & no C & no C & $\mathrm{C}$ & freon(1.0atm) \\
10.0 & T1,T2 & no C & no C & $\mathrm{C}$ & freon(1.0atm) \\
14.6 & T1,T2 & no C & no C & C & freon(1.0atm) \\
\hline
\end{tabular}

The calibration was performed in April 1991, when AGS SEB proton beam was available to E814. The setup for beam calibration is shown in Figure 18. It consists of a vertex detector(VTX1), a pair of beam scintilator horoscopes(S2,S4), a Cherenkov counter(C), two $1.0 \mathrm{~cm}$ diameter $\mu$ scintilator counters $(\mathrm{T} 1, \mathrm{~T} 2)$ in both front and back of the PCAL, 
and a 12 inch paddle scintilator(T3) at the end. Two drift chambers were also turned on for beam momentum test. Table 3 shows the identifications of different particles at different momenta.

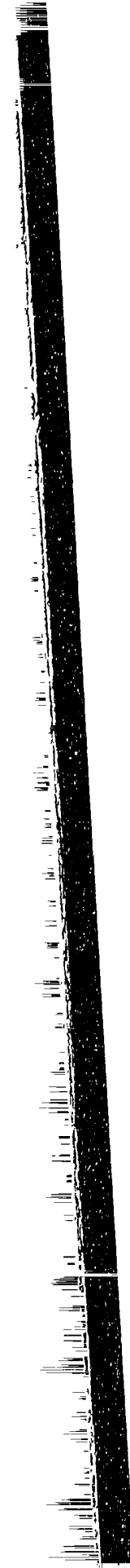

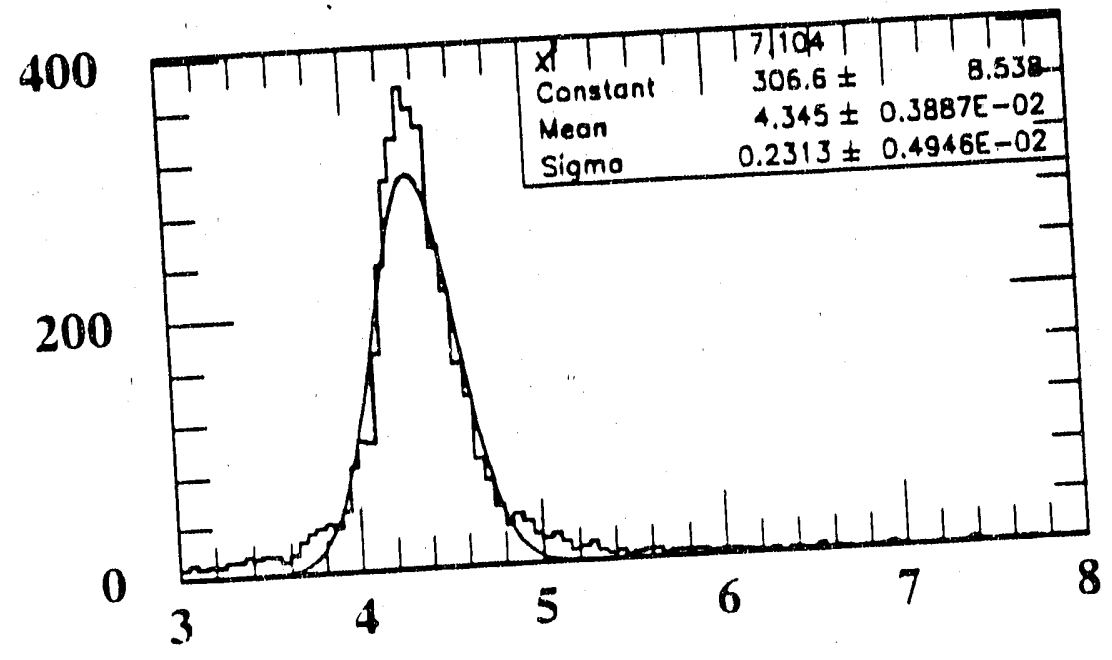

Figure 19: Momentum of the beam used in the PCAL calibration, obtained from tracking in the E814 magnetic spectrometer.

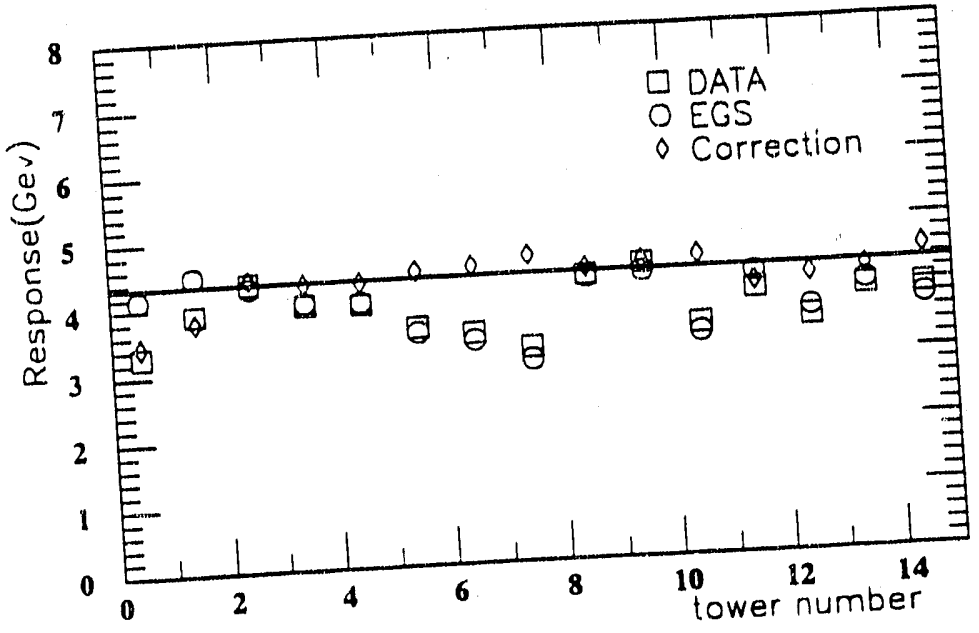

Figure 20: Mean values of the electron energy for a beam of $4.31 \mathrm{GeV} / \mathrm{c}$ (squares). Monte Carlo simulated data are shown as circles. Corrected data are shown as diamonds.

Figure 19 shows the beam momentum at a nominal value $4.31 \mathrm{Gev} / \mathrm{c}$, the histogram 


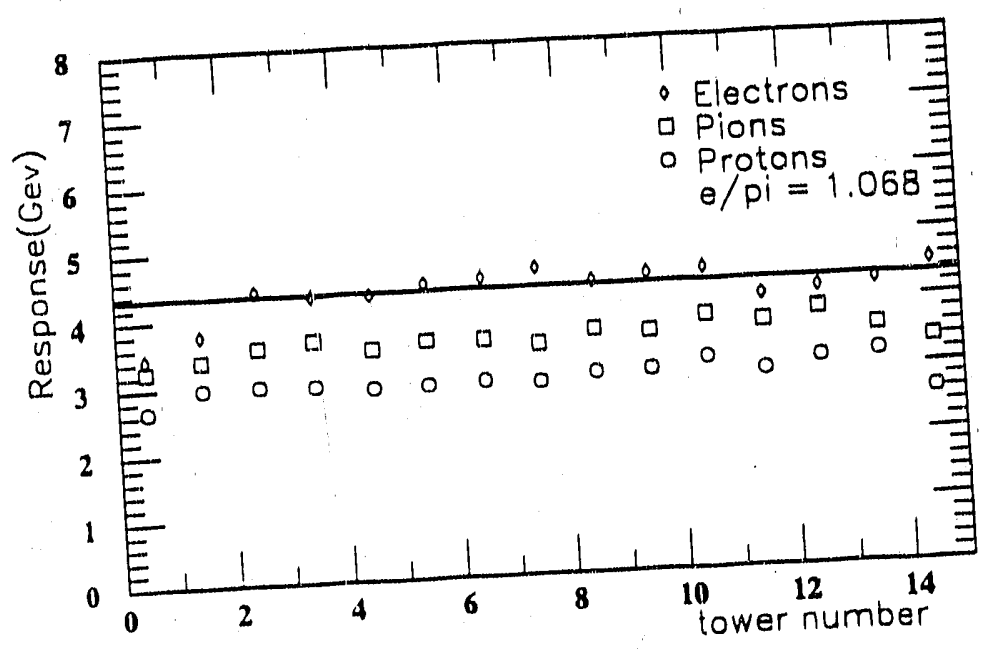

Figure 21: Comparison of electron, pion and proton responses for a beam of $4.31 \mathrm{GeV} / \mathrm{c}$. From these data we obtain a value of the $e: \pi$ ratio of 1.07 .

is the measured value using drift chambers and GRIST track reconstruction code. All towers in pie 16 and towers of the: 2 smallest sizes in quadrant IV(shown in Figeure 17) were calibrated at this energy. For each tower, a tape of about 8,000 events were recorded with mixed trigger of $\mu$, electron, $\pi$ and proton.

Relative calibrations of the photomultinliers for these towers in the beam are achieved using $\mu$ data at $4.31 \mathrm{Gev} / \mathrm{c}$. The energy loss of $\mu$ 's while traversing the calorimeter is essentically minimum ionizing and can be calculated basing on the Bethe-Bloch formula to set the relative calibrations for all towers seen by the beam.

Figure 20 shows electron energy depositions in all these calibrated towers, with relative gain matching from $\mu$ data. The large fluctuations are due to the fact that optical coupling varies drastically from plate to plate and from tower to tower. By putting in the coupling constants obtained from ${ }^{60} \mathrm{Co}$ source scan, EGS simulation tracks these fluctuations to within $5 \%$, except the first two towers because of the leakage into the PCAL center hole. Average electron response is then corrected by taking out the effect of optical coupling.

Pion and proton responses were also measured and Figure 21 shows them together with electrons. Taking the average of size $4,5,6$, where there is minimal side leakage, 

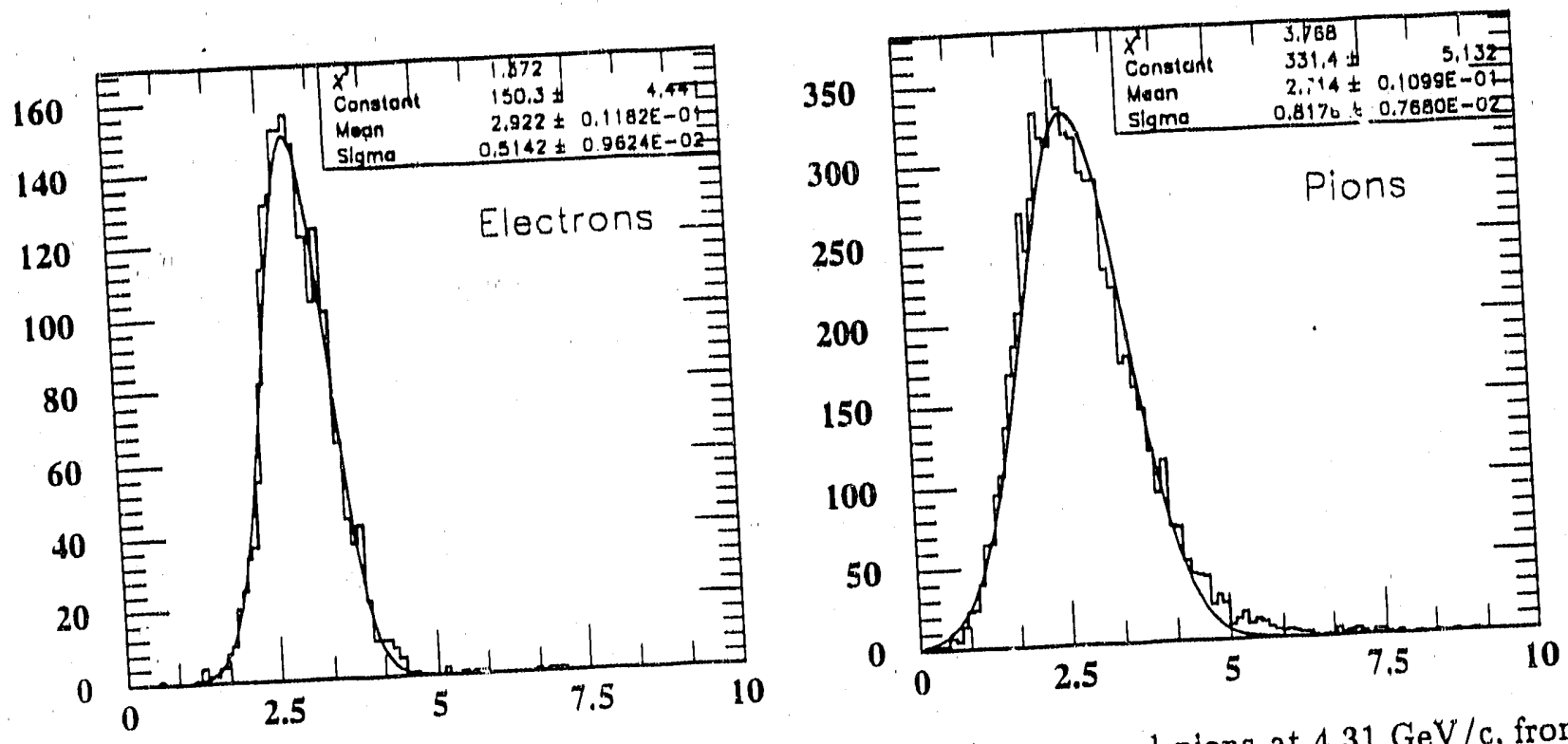

Figure 22: Response curves of a single tower $(5,16)$ to electrons and pions at $4.31 \mathrm{GeV} / \mathrm{c}$, from which the resolution values of the calorimeter are obtained.

the $\mathrm{e} / \pi$ response ratio is about 1.068 if the back leakage is considered as $6 \%$ as in the literature [12].

Figure 22 shows typical responses of the calorimeter to electron and $\pi$ beams. In this case, beams are aimed at the center of tower $(5,16)$. The resolution for electromagnetic shower is $28 \% / \sqrt{E}$ averaged among the calibrated towers while hadronic shower resolution is $50 \% / \sqrt{E}$. These resolution data will be put into a parametrization Monte Carlo program PROPHET simulating the calorimeter response.

\subsubsection{Trigger}

The design of the PCAL trigger system is that towers in every azimuthal segment are grouped into two fast analog sums on the side of the calorimeter, one for electromagnetic sections and one for hadronic sections. Each tower is weighted according to its geometry to form a analog sum of $\mathrm{E}_{T}$ in that group. 32 analog sums are thus generated and being transmitted via fast cables to the counting house where trigger decisions are made before taking the event.

The trigger system of PCAL for the measurement of tranverse energy has been im- 


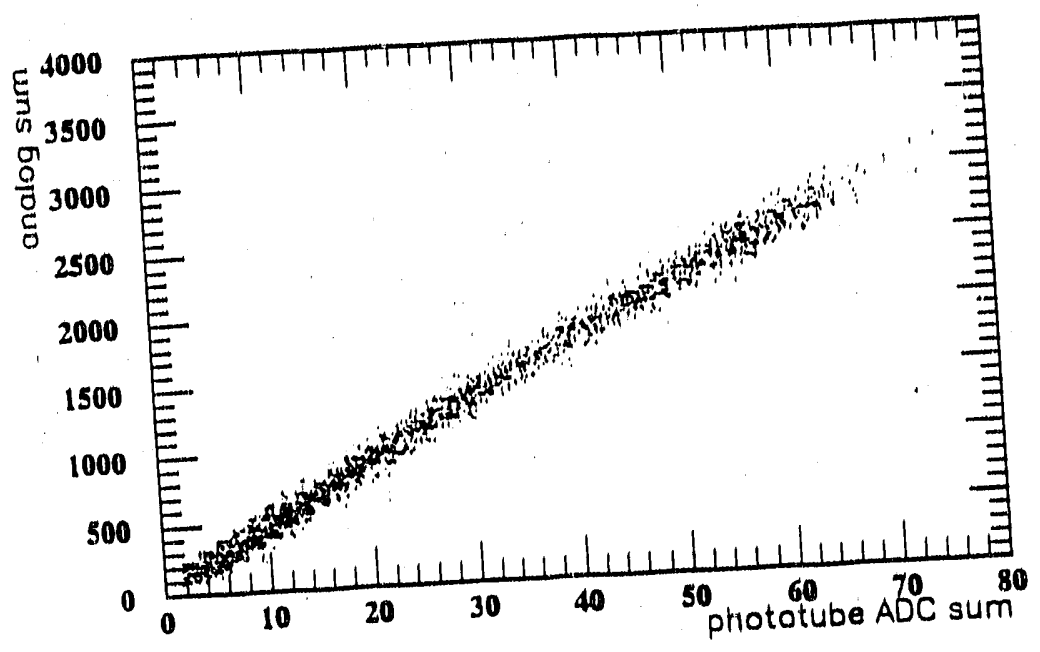

Figure 23: Trigger ananlog sum of PCAL $E_{T}$ versus the corresponding digital sum of all indlvidual towers with proper geometrical weights.

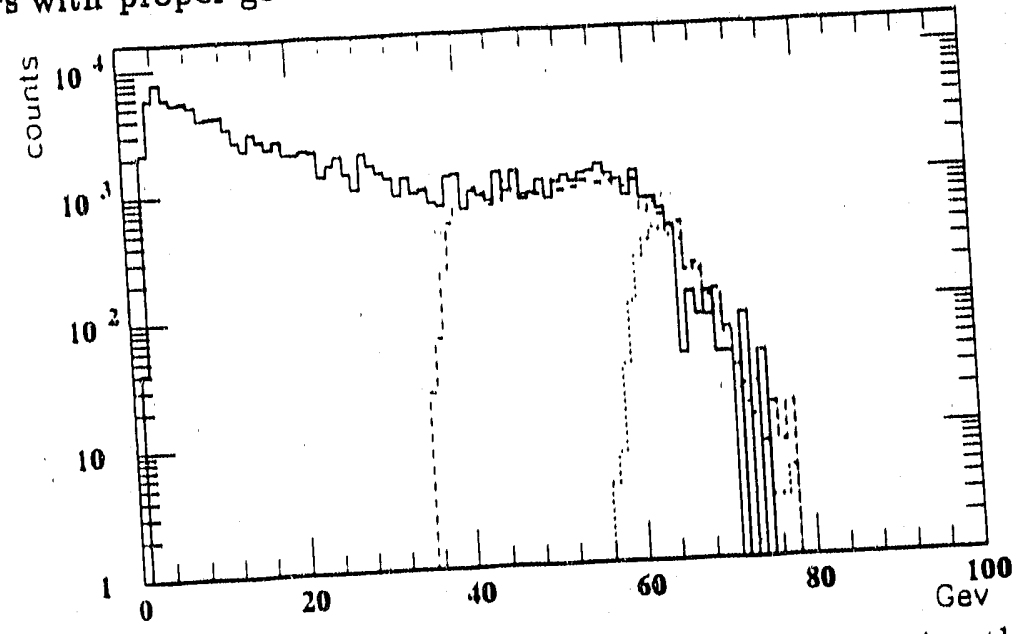

Figure 24: Online $E_{T}$ distribution showing triggering thresholds

proved before the ' 91 run. A number of errors in the trigger electronics had been corrected at the Pitt electronics shop and the system was tested before the run with a test pulse setup to insure its being operational during the run. In Figure 23 is the correlation between the analog sum of the total $E_{T}$ and the digital sum of all the phototube readouts. The linearity and width of the correlation shows the reliability of the trigger analog sum. Figure 24 indicates the positions of three $E_{T}$ trigger thresholds. Downscaling factors for these three levels are 41,8 and 1 . 


\subsubsection{Calorimeter Response simulation}

The program to simulate the calorimeter response is PROPHET, written first by Dagan and Oren for the Helios experiment at CERN. Instead of tracking every generated particle as the shower develops, PROPHET speeds up the calculations by parametrizing the shower development, both longitudinally and laterally. Such a parametrization is ob. tained from experimental results from calorimeter tests. It is, as expected, different from one type of calorimeter to another. The parameters used for PCAL simulation have been tuned for its showers size, $\mathrm{e} / \pi$ ratio and mip/e ratio etc. to best fit the detector response to various beams tested in the SEB run.

Parametrization is based that of R.Bock el al[13]. The starting point of the shower is calculated in the GEANT frame, based on various intercation cross-sections. The development of the electromagnetic showers is characterised by the radiation length of the media,

$$
d E=E * C * t^{\alpha-1} * e^{-\beta \omega t} * d t
$$

where $C$ is the normalization constant, $t$ the depth in radiation length and $\alpha, \beta$ as parameters to fit.

Hadronic showers are parametrized with two parts, one with radiation length and one with absorption length of the media,

$$
d E=E * C *\left(w * t^{(a-1)} * e^{-b * t} * d t+(1-w) * s^{(c-1)} * e^{-d * s} * d t\right)
$$

where $w$ is the portion of the shower characterized by radiation length, $t$ in radiation length, $s$ in absorption length and $a, b, c, d$ are parameters to be tuned. The parameters usually have a logarithmic energy dependence. To speed up computation, the above distributions as a function of $E, t$ and $s$ are calculated in the initialisation stage and stored in a table. 


\subsubsection{Data Analysis}

In Febuarary 1991, a week of running time was dedicated to transverse energy $\left(E_{T}\right)$ mea surements with participant calorimeter as the principal instrument. It was the first time this detector was used as a self-triggering device.

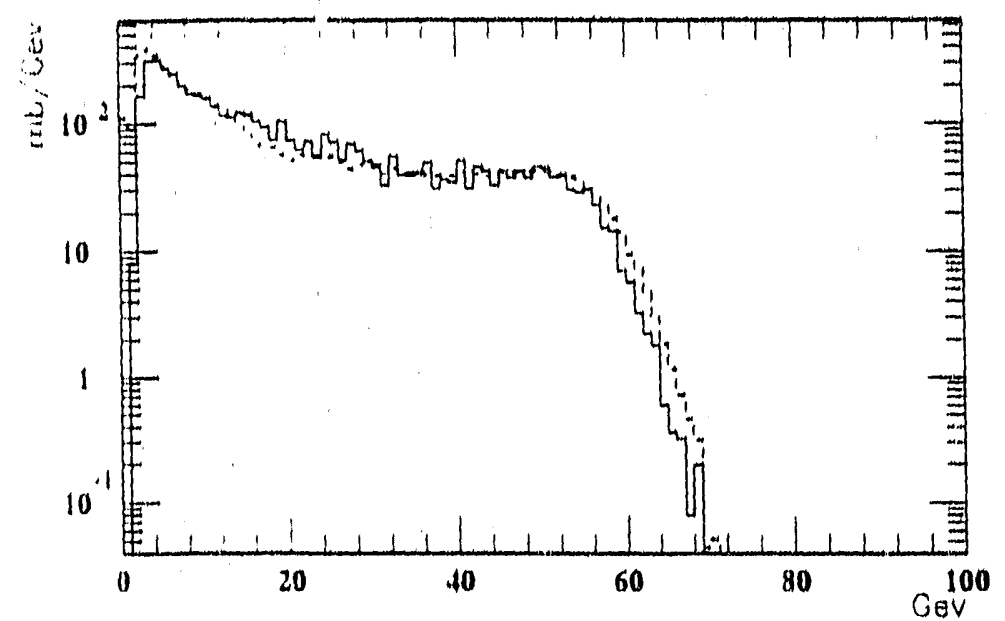

Figure 25: Raw $E_{T}$ distribution from the 1991 (solid curve) and 1990 (dashed curve) data. The two sets of data utillze two independent callbrations.

The calibration from the SEB run was tranfered back to Febuarary heavy lon data by comparing the ${ }^{60} \mathrm{Co}$ scans at the two times and then spread out to the entire detector using high multiplicity $(>100)$ heavy ion data, following the procedure in section 3.2.2. In Figure 25 is the raw transverse energy detected in the PCAL region. For consistency, also shown is the same spectrum from June 1990, with a set of totally independent calibration constants.

We are developing a mechanism to unfold raw data to real transverse energy distribution. A matrix $(M)$ is built to fold a certain $E_{T}$ distribution $(\alpha)$ (frorn Monte Carlo) into simulated detector response $(D)$,

$$
M \alpha=D
$$

where $\alpha$ is an array of $8 \mathrm{~d} E_{T} / \mathrm{d} \eta$, binned as in Figure 26. $D$ is an array of 32 elements, each representing the sum of $E_{T}$ in towers with the size and same depth(a ring). Each 


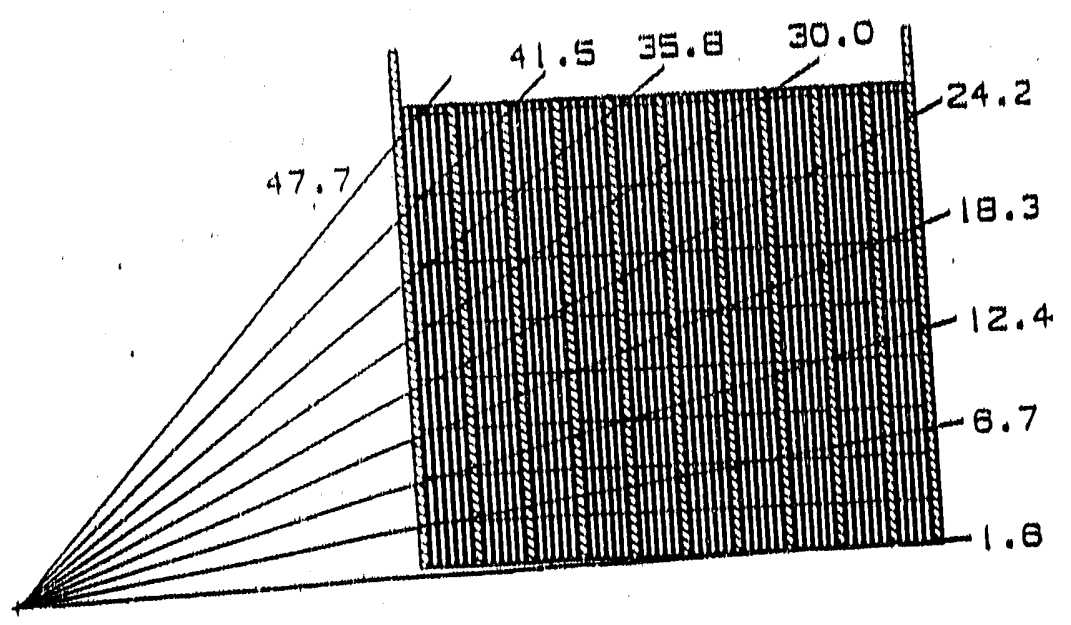

Figure 20: Dlagram showing a side view of the upper half of PCAL indicating the pseudorapidity blns used in the analysis. The beam is Incldent from the left, and the target is located at the point marked $(t)$.

element of matrlx $M$ is then naturally the percentage of $E_{T}$ deposited in a particular ring from a certain $\eta$ bin.

When applying the matrix to raw data(real detector response), a least $\chi^{2}$ approach is used, $\alpha$ is treated as a set of parameters to be tuned so that

$$
\chi^{2}(\alpha)=\sum_{i} \frac{\left(D_{i}-M_{i j} \alpha_{j}\right)}{\sigma_{i}^{2}}
$$

can be minimized. The fitted $\alpha$ is then expected to be the real $E_{T}$ distribution. By taking into consideration the depth information and tower to tower correlation due to shower spread, the model dependence on the Monte Carlo $E_{T}$ distribution is expected to be minimized.

To examine model (in)dependence of this method, FRITIOF and HIJET Monte Carlo are employed. $E_{T}$ distributions of both models are folded into simulated detector response by PROPHET, and two matrices are built accordingly. Figure 27a compares the original and unfolded $E_{T}$ distribution of FRITIOF, and shows the sensitivity of the unfolding method to different models. Figure 27b makes similiar comparison for HIJET.

In Figure 28 raw data are unfolded into total transverse energy distribution in PCAL's pseudorapidity region and compared with that of FRITIOF. It falls short of the simulation, 

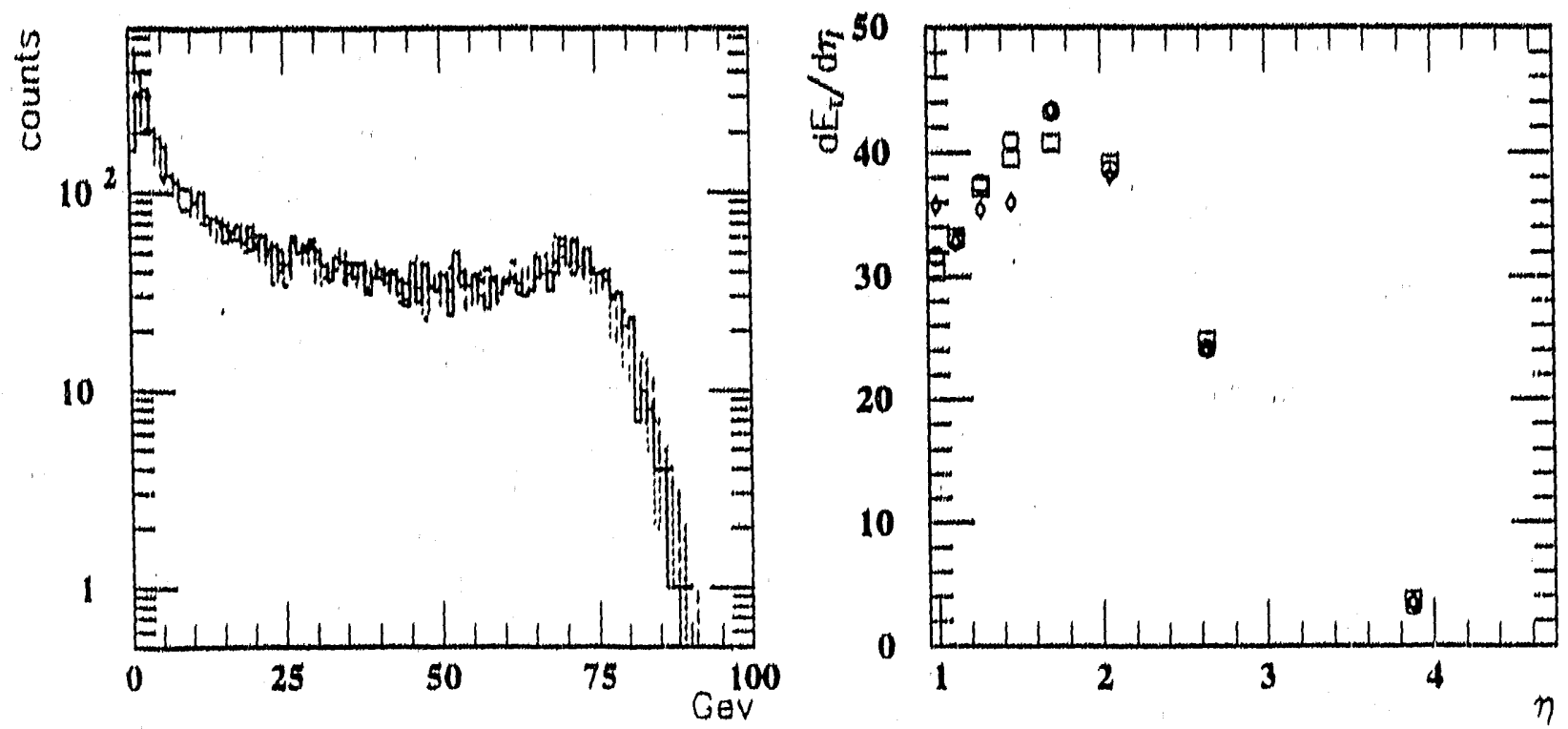

(a)
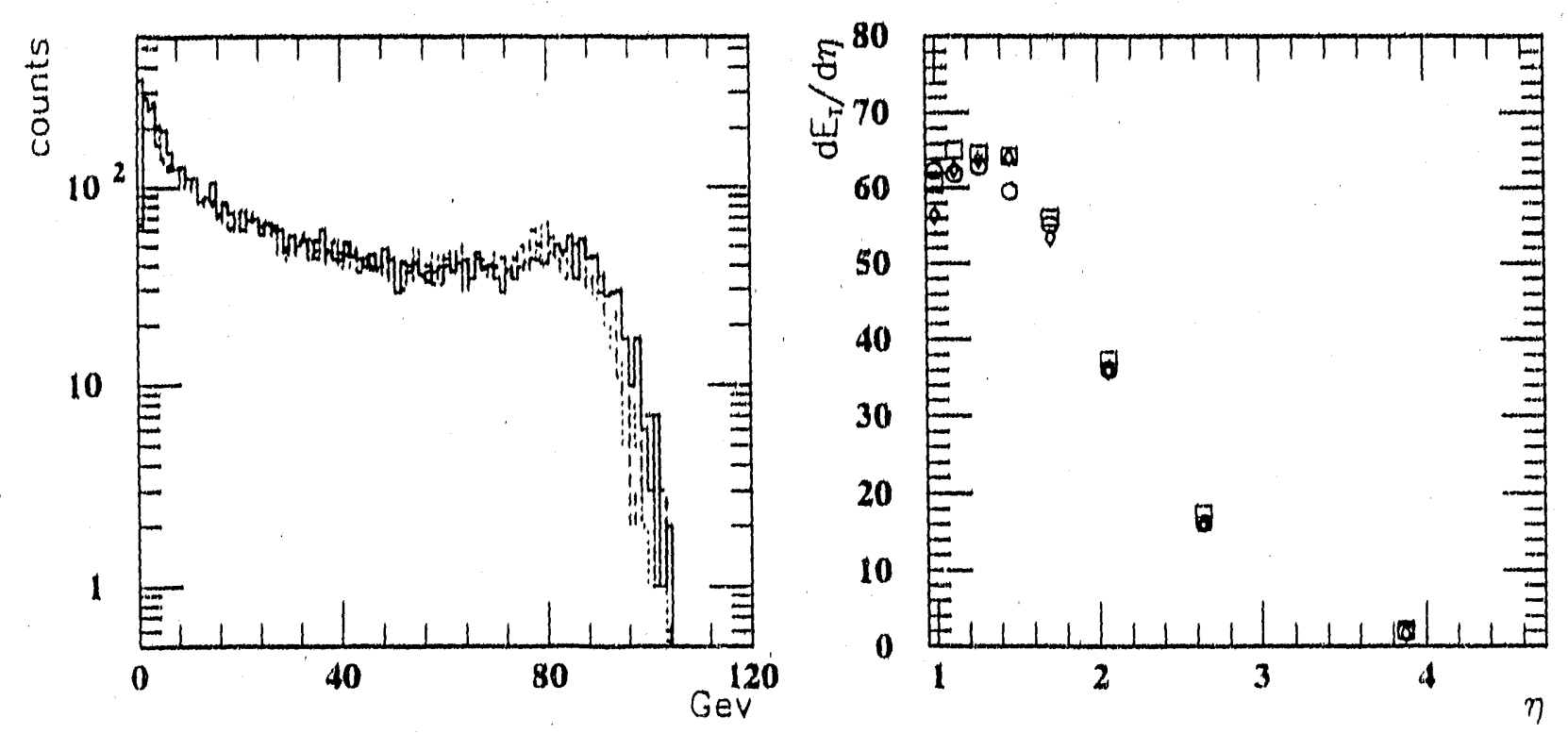

(b)

Figure 27: (a)FRITIOF simulation of the total $E_{T}$ distribution(solid curve on the left) and $\mathrm{d} E_{T} / \mathrm{d} \eta$ (squres on the right), compared with the corrected values obtained from the unfolding procedure using matrices derlved from FRITIOF(dotted curve and cirles) and HIJET(dashed curve and diamonds). (b)The same test for HIJET 


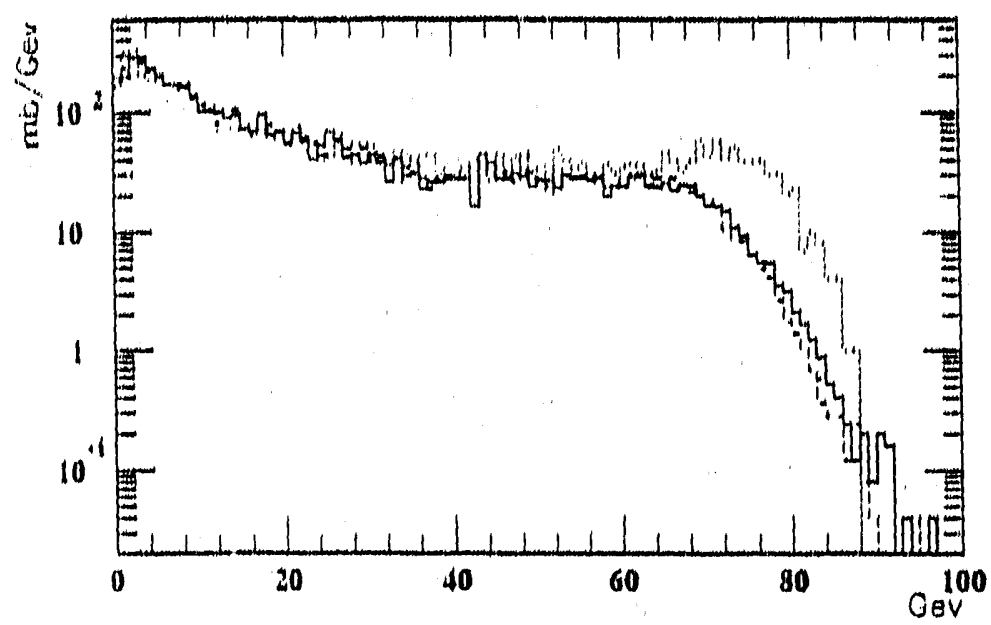

Figure 28: Total $E_{T}$ distribution obtained using correctlon matrices derived from FRITIOF(dashed) and HIJET(solld), Dotted curve is FRITIOF simulation

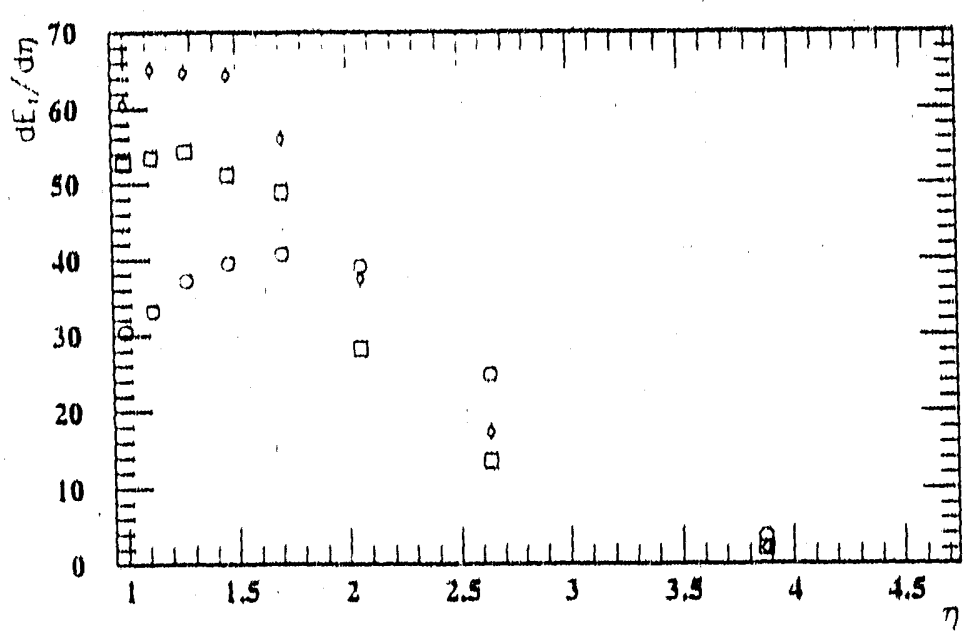

Figure 29: Values of $d E_{T} / d \eta$ obtalned from the unfolding process using the matrix $M_{F R}$ obtained from FRITIOF (open squares), compared with the generated values from FRITIOF (circles) and HIJET (diamonds). 
and the cutroff is not as sharp as that of the simulation. The model independence of the unfolding is clearly shown when matrices generated both with FRITIOF and HIJET are applied to data and the results are almost identical.

Figure 29 compares the $d E_{T} / d \eta$ distribution to those of FRITIOF and HIJET. At large angle data are in between but at small' angle data fall below both FRITIOF and HIJET.

\section{Personnel}

1. W. E. Cleland, principal investigator. Cleland's principal role in Experiment 814 has been in the area of organization of the trigger electronics. His efforts include planning of the trigger system, decisions regarding the purchase of colnumarcial units for the trigger, supervision of the modification of exlsting electronics, and the design of new electronics which must be constructed. He also was responsible for the integration of the detector systems which make up the experiment. During the past year his major activity has been involvement in the analysis activities of the Pitt group.

2. E. Marcia Takagui. Takagui is a postdoctoral research associate who joined us in December 1989. She is working in the area of peripheral collisions and is presentiy concentrating on the the reconstruction of $2 \mathrm{p}$ data, for which she is responsible. In addition she is system manager for our Silicon Graphics based computing system.

3. D. E. Kraus. Kraus is a part-time instructor at the University of Pittsburgh with considerable experience in experimental high energy physics. He has spent approxi- 
years 1987-90. His principal technical responsibillties in E814 have deen un suuusuo the behavior of photomultiplier tubes in the environment of heavy fon experiments; design, construction, calibration and maintenance of the forward and magnet scintillators; and development of a DC light source for use in the participant and beam calorimeters. Currently he is spending a small fraction (1/6) of his time participating in the analysis of the E814 data.

4. S. Voloshin. Voloshin is a theorist at the Moscow Engineering Institute who became interested in our multiplicity data and spent two months at Pitt as a visiting scientist. He worked with Jayananda on the analyis of the two particle correlation functions and was in important source of encouragement and theoretical guldance,

5. K. Jayananda, Jayananda was a graduate student who carried out his thesis research in central collisions. His first effort in this experiment, carried out during the summer and fall of 1987, were to analyze the data for albedo yields taken during the May 1987 test run. He then took responsibility for the design and commissioning of the silicon multiplicity detector, and helped to develop the summing electronics to integrate the information from the multiplicity detector into the second level trigger. His thesis, Appendix B of this report, was finished in October 1991, and included several studies of the charged multiplicity data. One of the more interesting topics he studied is the two particle correlation functions, made possible by the high statistical accuracy of our data. He now holds a faculty position at the University of Sri Jayewardenepura in Sri Lanka and plans to continue to collaborate on the two-particle correlation analysis.

6. Bo Yu. Bo Yu was a graduate student who carried out a thesis in high energy physics in instrumentation under the supervision of V. Radeka of BNL. He was responsible for the detailed design of the prototype for DC1, a novel type of chamber using interpolative readout of cathode pads. He was then heavily involved in the design 
and testing of the prototype chamber and had followed through with the design, testing, and installation of the final chamber. He also designed the pad planes for DC2 and DC3. His thesis, finished in December 1991, was a study of drift chambers with interpolative cathode pad readouts. He currently holds a research position at Brookhaven National Laboratory.

7. U. Sonnadara. Upul Sonnadara is a graduate student who has been working with us since the summer of 1988 . He passed the comprehensive examination in May, 1989. He has helped with the assembly and calibration of the forward scintillation counters, and he has written monitor programs on the data-acquisition VAX in order to monitor their performance. He has taken on the responsibility for the calibration of the forward and magnet scintillators. He is heavily involved in the peripheral data analysis and was the major author on the 1p paper incluaed in Appendix A. He plans to continue his thesis research with an analysis of the in data.

8. Z. Zhang. Zhang is a graduate student who joined our group in April, 1989, having passed the comprehensive exam in May, 1987. His principal responsibility is for the data taken with the participant calorimeter, so during the past year much of his effort has been spent on the calibration and gain matching of this device, including a and a study of the triggering electronics. In addition Zhang built the trigger monitoring workstation based on expert systems techniques, installed in E814 before the June 1990 run, and he continues to maintain this device. He plans to write a thesis in the area of central collisions, concentrating on data from the participant calorimeter.

9. Tomas Joyce. Joyce is an undergraduate engineering student who has been working with our research group on a part time basis since the spring of 1991. He has carried out a number of data analysis tasks, including studies of the gains of photomultiplier tubes required for the peripheral analysis and studies of neutron calorimeter using calibration data taken with a proton beam.

10. Brian Lopresti. Lopresti is an undergraduate physics major who has been working 
with our group on a part time basis since the spring of 1991 . He has been helping with analysis tasks, including studies of the timing properties of the time of flight counters installed in the experiment in 1990 . He is currently involved in the analysis of peripheral collisions with $\alpha$ particles in the final state.

11. J. Rabel. Joe Rabel is an electronics engineer in our group who who has spent considerable effort on the E814 electronics during the past several years. His expertise in digital logic has been invaluable in carrying out the design and testing of the CAMAC modules needed for our trigger processor. Mr. Rabel also maintains the electronics modules which are used in E814, including the large number of current monitors used for calibrating the calorimeters. Currently he is involved in work connected with our RHIC R\&D project, in which field programmable gate arrays are being evaluated for their usefulness in triggering applications. 


\section{References}

[1] B. Bassalleck, et al. Z. Phys $C 38$ (1988) 45

[2] J. Barrette et al. Phys. Rev. Letters 64 (1990) 1219.

[3] J. Barrette et al. Phys. Rev. C 41 (1990) 1512.

[4] J. Barrette et al. Phys. Letters B252 (1990) 550.

[5] J. Barrette et al. Phys, Rev, C, to be published.

[6] J. Barrette et al. "Excitation Energy Distribution of Relativistic ${ }^{28} \mathrm{Si}$ in Electromagnetic Dissociation into $\mathrm{p}+{ }^{27} \mathrm{Al}$ ", submitted for publication in Phys. Rev. $C \operatorname{Dec} 1991$.

[7] J. Barrette et al. "Charged particle multiplicity in ${ }^{28} \mathrm{Si}+\mathrm{Al}, \mathrm{Cu}$ and $\mathrm{Pb}$ reactions at $E_{l a b}=14.6 \mathrm{GeV} /$ nucleon", submitted for publication in Phys. Rev. C Nov 1991.

[8] J. Fischer at al., IEEE Trans. Nucl. Sci., NS-37 (1990).

[9] R. Debbe at al., IEEE Trans. Nucl. Sci., NS-37, (1990).

[10] Bo Yu "Gas Proportional Detectors with Interpolating Cathode Pad Readout for High Track Multiplicities", Ph.D. thesis, University of Pittsburgh, December 1991

[11] J. Lissauer and H. Takai, Phys. Rev. C 41 (1990) 2410.

[12] M.G. Catanesi at al, Nucl. Inst. and Methods A260(1987) 43-54

[13] R.K. Bock at al, Nucl. Inst. and Methods 186(1981) 533-539

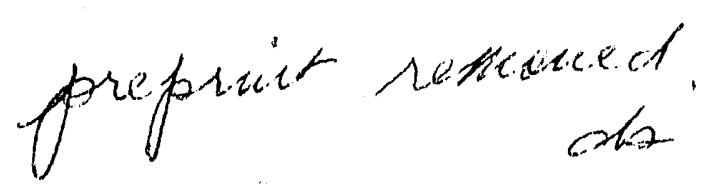




\title{
Studies of Relativistic Heavy Ion Collisions at the AGS (Experiment 814)
}

\author{
W. E. Cleland \\ University of Pittsburgh \\ Department of Physics and Astronomy
}

\section{DISCLAIMER}

This report was prepared as an account of work sponsored by an agency of the United States Nerther the United States Government nor any agency thereof, nor any of their Government. Neither the United States Go implied, or assumes any legal liability or responsiemployces, makes any warranty, express or implied, or assumes any logal tiability or reduct, or bility for the accuracy, completeness, or usefulness of any information, apparat rights. Referprocess disclosed, or represents that its use would not infringe privately owned rights. Reference herein to any specific commercial product, process, or service by trade norsement, recommanufacturer, or otherwise does not necessarily constitute or imply its enderef. The views mendation, or favoring by the United States Government or any agency theref. The the and opinions of authors expressed herein do not

United States Government or any agency thereof.

Annual Progress Report

DOE Grant No. DE FG02 87ER40363

1 May 1991 - 30 April 1992 


\section{Studies of Relativistic Heavy Ion Collisions at the AGS (Experiment 814)}

During the past year, the Pittsburgh group has continued to work with the E814 collaboration in carrying out AGS Experiment 814. We present here a brief history of the experiment, followed by a detailed report of the analysis work being pursued at the University of Pittsburgh.

In May 1987, during the heavy ion run at the AGS, we mounted a test setup, which was used (a) to understand the behavior of the modified uranium/scintillator calorineters, (b) to test the prototype forward scintillation counters, (c) to carry out a set of albedo measurements, and (d) to carry out a measurement of the $E_{T}$ spectrum for a $10 \mathrm{GeV} /$ nucleon beam incident upon a variety of nuclear targets. Preliminary results [1] from this run indicate that there is almost complete stopping of the ions at $10 \mathrm{GeV} /$ nucleon, as the limit in $E_{T}$ reached seems to depend little on the A of the target nucleus. In November 1987, we mounted a smaller test in order to study the properties of scintillation counters in relativistic ion beams. In December 1988, the apparatus in its final configuration (but still incomplete) was exposed first to proton beams, in which debugging and testing was carried out, and then to $15 \mathrm{GeV} /{ }^{28} \mathrm{Si}$ beams, during which a significant data sample was taken on each of the three major physics topics we are studying. The 1988 data have been analyzed, resulting in the publication of three papers $[2,3,4]$.

In June 1989, another run was carried out, in which all of the forward calorimeters were in place, while the drift chambers and the participant calorimeter were being tested. Results from this run on central collisions have been recently published [5]. Another ${ }^{28} \mathrm{Si}$ run was carried out in June 1990, in which the apparatus was finally complete, including the participant calorimeter, the drift chambers, and a new device, the beam vertex detector. There was a strong emphasis on peripheral data in this run, and a paper on $1 p$ emission has recently been submitted for publication [6] (included here as Appendix 
A). Other peripheral channels are still being analyzed, and the progress in this area is one of the main topics of this report. In addition, a set of data on central collisions were taken using the silicon multiplicity detector as the trigger. The analysis of these data was the main topic of the Ph.D. thesis of M. K. Jayananda, included here as Appendix B. In addition, a paper on the charged multiplicity distribution has been submitted for publication [7]. In 1991 we carried out a calibration run on the participant calorimeter as well as a data run on central collisions, with PCAL as the main trigger. The status of the analysis of these data is described in detail below. Another topic in the 1991 run was a measurement of the $p_{T}$ spectra of forward-emitted particles, which is being analyzed by the Stony Brook group.

As originally proposed, Experiment 814 is a study of both extreme peripheral collisions and the transition from peripheral to central collisions in relativistic heavy ion-nucleus interactions. We are studying relativistic heavy ion interactions with nuclei in two types of collisions: (a) extreme peripheral collisions of large impact parameter, and (b) central collisions with high transverse energy in the final state. The experiment emphasizes the measurement of overall event characteristics, in particular energy flow measurements and a precise measurement of the particle charge, momentum, and energy in the forward direction. This permits measurements of cross sections and rapidity densities as a function of the transverse energy for leading baryons emitted into regions of larger rapidity. Combining the energy flow measurements as a function of rapidity with the spectra of leading baryons provides information on the impact parameter dependence of the nuclear stopping of the projectile in relativistic heavy ion collisions. In 1988, the scope of Experiment 814 was enlarged to include a search for strange matter in central collisions, the first results of which have been published [4], and analysis on a longer run taken in 1990 is still under way. 


\section{Detectors in Experiment 814}

A diagram of the experiment is shown in Figure 1. We give a brief description of each of the detector systems below. More detailed information is available in our previous annual reports and in the publications and conference reports of the experiment.

- Beam vertex detector. The beam vertex detector is a pair of silicon strip detectors used to locate the horizontal position and angle of the incoming beam particle. This system was installed before the June 1990 run, in order to improve the resolution in the center of mass quantities in certain exclusive peripheral interactions.

- Beam counters. The beam detectors serve to define the presence of a beam projectile, to establish the time of the collision, and to identify the charge of the projectile. The telescope consists of two counters in coincidence and two other counters, with a hole for the beam, in anticoincidence. The geometrical coincidence signifies that a track has crossed the target. A pulse height measurement from one of the counters is used to establish the charge of the projectile, and the timing of the other counter is used to establish its time of arrival. The problems posed by large dynamic range in making good amplitude and timing measurements for scintillation counters operating in a heavy ion beam are nontrivial. Tests on the suitability of various scintillators and phototubes were carried out in a heavy ion run during the fall of 1987 . On the basis of these tests, NE102 was chosen as the scintillator material, Hammamatsu R2083 as the phototube for good timing, and EM 9954 as the tube for good charge resolution.

- Target. The target for E814 is a simple disk target with a remote positioning control. The rotating target assembly was built by the McGill group and was installed in the mount which supports the multiplicity counter. The disk has eight positions, so that up to seven targets plus an empty frame may be used at any one time. The complement of targets is changed for the different programs, as peripheral, central, and strangelet running require different materials and thicknesses. 


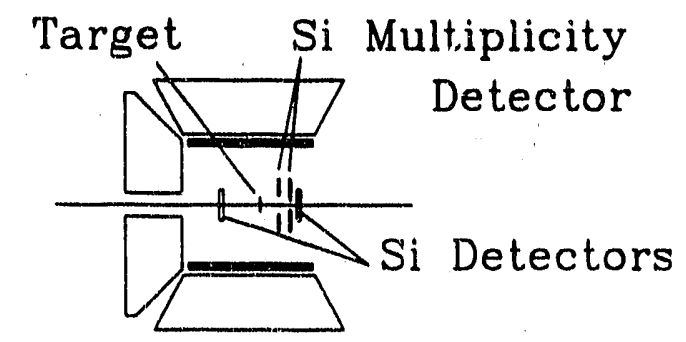

Vertex 1 Vertex 2
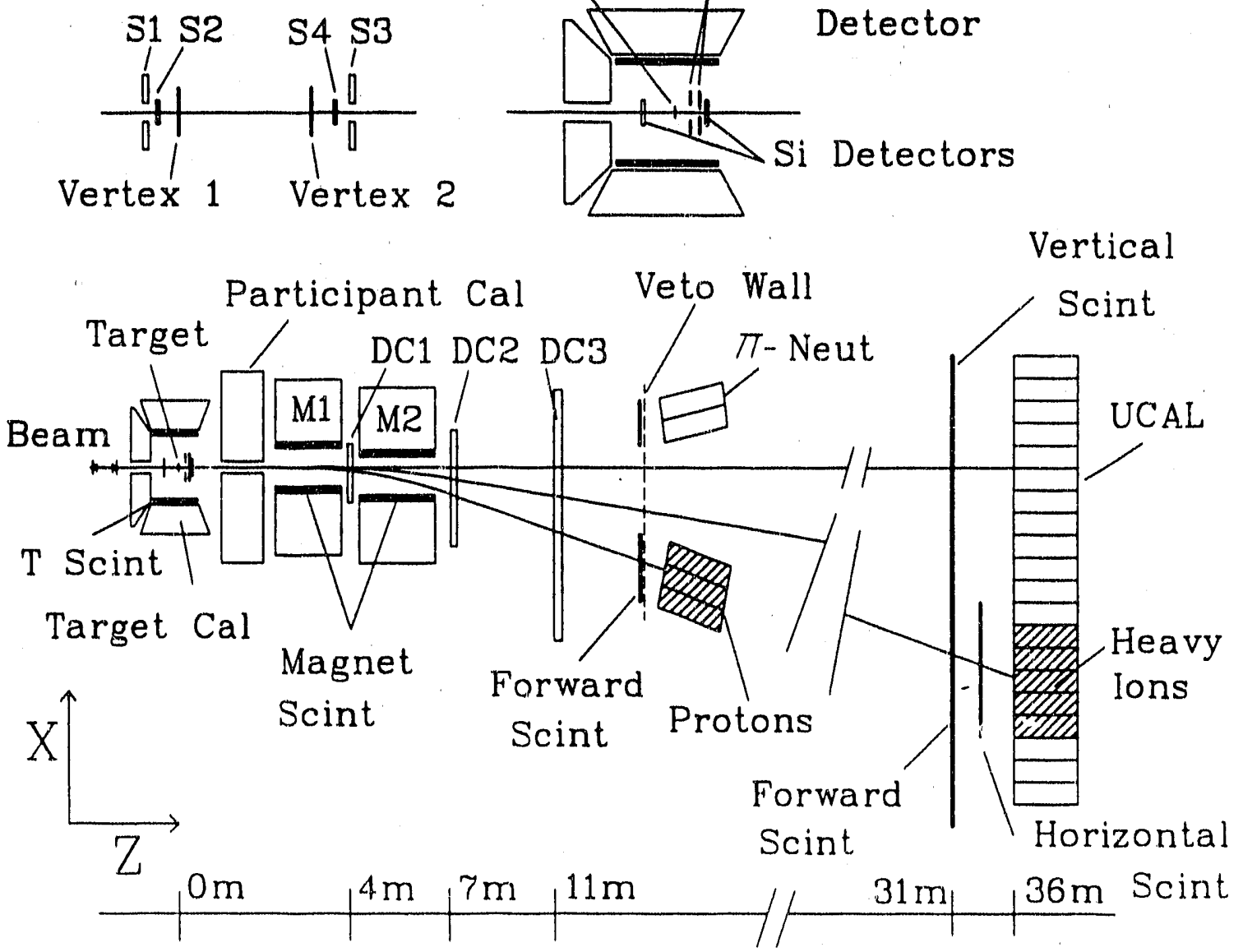

Figure 1: Diagram of the apparatus of Experiment 814. M1 and M2 are dipole magnets. DC1, DC2, and DC3 are drift chambers used to track particles in the forward spectrometer. Solid lines in the figure represent trajectories for neutral particles, beamlike particles (with $\mathrm{Z} / \mathrm{A}=$ $1 / 2$ ) and protons with $14.6 \mathrm{GeV} / \mathrm{c}$ momentum. 
- Multiplicity Detector. The silicon multiplicity counter consists of two 512 pad silicon detectors which are positioned immediately downstream of the target. They are used both at the trigger level and in offline studies of the multiplicity as a function of rapidity. This detector was fabricated at BNL, and the electronics was supplied by BNL, CERN, and New Mexico. K. Jayananda, a Pitt graduate student, was responsible for the pad layout and for designing the electronics for integrating it into the trigger. The detector was used successfully as a multiplicity trigger in the June 1989 run, and it was the source for most of the central triggers for the 1990 and 1991 runs. Details of the detector and analysis of the multiplicity data are given in Jayananda's Ph.D. thesis, included here as Appendix B.

- Target Calorimeter. The target calorinıeter is used to detect the products of the target fragmentation and covers the region of $\theta$ between 45 and 180 degrees. It consists of a box composed of 900 blocks of $\mathrm{NaI}, 4 \times 4 \mathrm{~cm}$ in cross section and 6 radiation lengths thick. It serves to measure $E_{T}$ in central collisions and it also acts as a veto for the peripheral collisions. The NaI is read out using photodiodes followed by prearnplifiers and shaping amplifiers. Summing electronics for the first and second level trigger has been designed and fabricated at Stony Brook. The construction and calibration of this detector is the responsibility of the Stony Brook group.

- Participant Calorimeter. The participant calorimeter, constructed by Los Alamos and Texas $\mathrm{A} \& \mathrm{M}$, is azimuthally symmetric, covering the polar angle region $1.6^{\circ}$ $<\theta<48^{\circ}$. It has both azimuthal and polar angle segmentation. The radiator is lead and iron plates, and the sampling layer is plastic scintillator, coupled to phototubes via wavelength-shifting fiber light guides. The electromagnetic and hadronic sections of the calorimeter are read out separately. Fast trigger sums, using $E_{T}$ weights, are formed separately for each azimuthal section, both for the electromagnetic and hadronic sections of the calorimeter. Z. Zhang, a Pitt graduate student, is responsible for the PCAL and its trigger electronics, including the problem of 
on-line and off-line gairs monitoring and adjustment. A discussion of the status of: the calorimeter and the data taken with the PCAL trigger is given in Section 3.2.1.

- Tracking Chambers. In order to measure the momenta of the charged particles emitted in the collisions, we use a spectrometer consisting of three sets of tracking chambers and two magnets. This configuration is chosen due to the wide range of particle momenta. One of the chambers, DC1, has highly segmented pad readout and interpolation by charge division. It is positioned between the two magnets. The chamber has 1000 pads, and the density varies across the plane in accordance with the expected track multiplicity. The design of this chamber was carried out in the Instrumentation Division of BNL. One of the key people in this group was Bo Yu, a graduate student at the University of Pittsburgh who has recently completed his Ph.D. thesis[10], which contains several studies relevant to the E814 tracking chambers, especially DC1. The other drift chambers for the experiment, DC2 and DC3, consist of six drift planes and two planes with cathode jad readout. They have been designed at BNL, with the fabrication of the frames carried out at Yale. These chambers were installed just before the June 1989 run and were fully operational in the June 1990 run.

- Forward Calorimeters. Charged particles with momenta greater than $6 \mathrm{GeV} / \mathrm{c}$ and all neutral particles emitted in a cone of $\theta$ less than 1 degree are measured in the forward calorimeters. In 1988, we had only 13 of these units, since they were obtained from CERN and were still required in HELIOS. The remaining 12 units were shipped to BNL in early 1989 and were modified and installed in the experiment before the June 1989 run, completing the set of uranium/scintillator calorimeters in the forward spectrometer. Just upstream of each of the calorimeter stacks are a bank of scintillation counters, two counters associated with each stack, which are used to measure the charge and the vertical position of the track. Because fragments emitted in the forward direction have approximately the velocity of the beam particle 15 $\mathrm{GeV} /$ nucleon, the energy measurement in the calorimeters is a good measure of the 
nucleon number. The use of calorimeters in the detection of final state neutrons is particularly attractive, as the good energy and spatial resolution of these calorimeters permit studies of correlations among leading neutrons in order to get information on transparency in nuclear collisions and to search for bound multineutron states. Thus is is important for us to understand the detailed response of these units to the forward emitted baryons.

- Scincillation Counters. There are three sets of scintillation hodoscopes used in the experiment: (a) the target counters, used in conjunction with the target calorimeter to identify charged particle frapments and neutrals; (b) magnet scintillators, which line the sidewalls of the two magnets, used to detect pions produced in the forward direction; and (c) forward scintillation counters, to be used in conjunction with the forward calorimeter to measure the charge of nuclear fragments. The target counters have been constructed and installed by the Stony Brook group. The magnet counters and forward scintillation counters have been constructed and installed by the Pitt group, and their operation and calibration is the responsibility of $\mathrm{Y}$. Sonnadara.

\section{Analysis of Peripheral Collisions}

\subsection{Data Sample}

In June 1990, during 10 days of running, we collected a large, high quality sample of data to study peripheral collisions. The recorded sample consists of two major parts, namely, (a) a single nucleon emission (1p-1n) and (b) two or three nucleon emission $(2 \mathrm{p}-2 \mathrm{n})$. These were recorded over 6 different targets including data taken with an empty target frame. In addition, a majority of the events were recorded with an online veto requirement to enrich the data sample. A small percentage of data were taken without the online veto requirement to study trigger efficiencies. In a normal data run, the trigger was set up to record, in addition to the peripheral events, a small fraction of events with minimal trigger requirements (pre-triggers), events with no interactions (beam triggers), 
Table 1: Sample used in the analysis of peripheral data.

\begin{tabular}{|c|c|c|}
\hline Target & $\begin{array}{c}\text { No of tapes for } \\
\text { single nucleon }\end{array}$ & $\begin{array}{c}\text { No of tapes for } \\
\text { two or three nucleons }\end{array}$ \\
\hline $\mathrm{Pb}$ & 8 & 36 \\
$\mathrm{Sn}$ & 7 & 36 \\
$\mathrm{Cu}$ & 3 & 37 \\
$\mathrm{Al}$ & 5 & 35 \\
$\mathrm{C}$ & 3 & - \\
$\mathrm{MT}$ & 4 & 33 \\
\hline
\end{tabular}

and random events with no beam particles present (empty events). These were used to study gain variations and other systematic effects. The recorded data sample is shown in Table 1.

In addition we recorded 18 beam trigger tapes, 18 pre-trigger tapes and 18 pulser tapes, also used för systematic detector studies.

These data were originally written into 6250 magnetic tapes (one tape for each run). The policy of the collaboration is to keep the original copy of the data at BNL and produce copies for individual institutions onto $8 \mathrm{~mm}$ tapes which can be read via hexabyte tape drives. Each $8 \mathrm{~mm}$ tape is used to record up to 12 runs from 6250 tapes. The peripheral sample with which we are working consists of 6 (one for each target) $8 \mathrm{~mm}$ tapes for $1 \ldots-1 \mathrm{n}$ triggers and 22 tapes for $2 \mathrm{p}-2 \mathrm{n}$ or rare triggers. This sample consists of about $2,400,000$ events.

\subsection{Event Selection}

In studying peripheral interactions, the main objective is to identify pure electromagnetic interactions from nuclear induced interactions. The detectors surrounding the target region are very useful for this. The Participant Calorimeter (PCAL) is an excellent tool 
for separaiting these two classes. In Figure 2 we show the energy seen by the PCAL for a normal peripheral run. The peak at $8 \mathrm{GeV}$ corresponds to events where one nucleon is emitted at an angle greater than 0.8 degrees (which corresponds to the aperture in the PCAI) due to high momentum transfer from nuclear induced collisions. Pure electromagnetic events do not produce detectable energy in PCAL, so we can set un upper limit of $1.0 \mathrm{GeV}$ (a typical noise level for the total energy sum) in PCAL as a yeto against nuclear interactions.

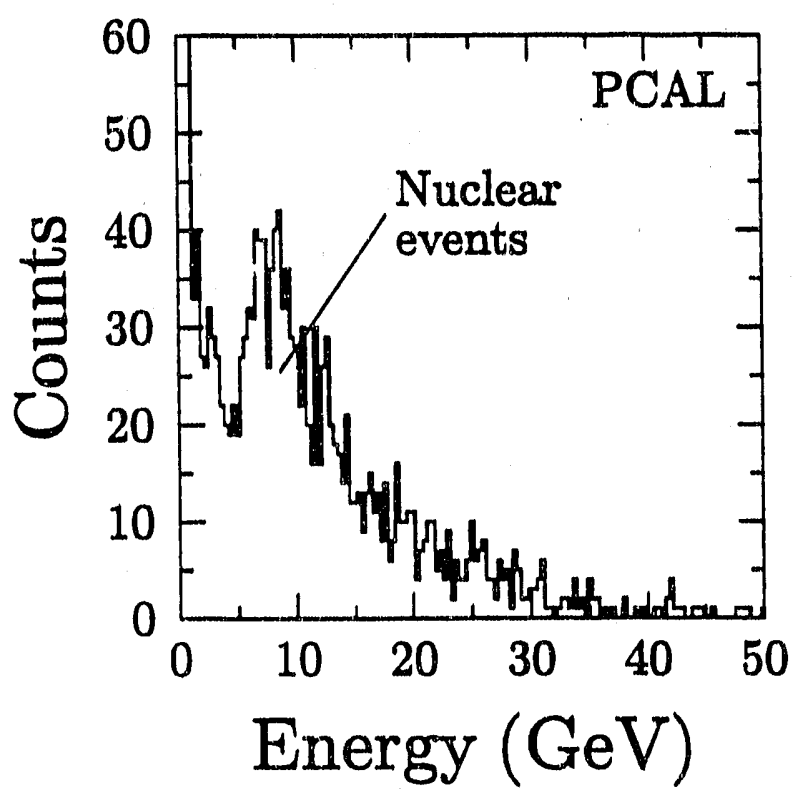

Figure 2: Energy deposited in PCAL in the peripheral data. The EMD events are charaterized by low $(<1 \mathrm{GeV})$ energy deposit in this calorimeter.

The second objective is to detect and identify all final state products, for which we used the downstream detectors. We identify the charge of the final state particles from the upstream and downstream scintillator hodescopes and energy from proton and neutron region calorimeters. Figure 3, (a) and (b) shows the measured charge distribution for heavy-ion fragments and the measured energy distribution for protous. We have achieved about $2.7 \%$ resolution in charge measurement and $0.6 / \sqrt{E}$ for energy measurement, both of which are adequate to identify low multiplicity peripheral channels (1p, $1 \mathrm{n}$ or $1 \mathrm{p} 1 \mathrm{n})$. The resolution in the charge measurement is good enough to separate all charges from 
$\mathrm{Z}=1$ to $\mathrm{Z}=14$.
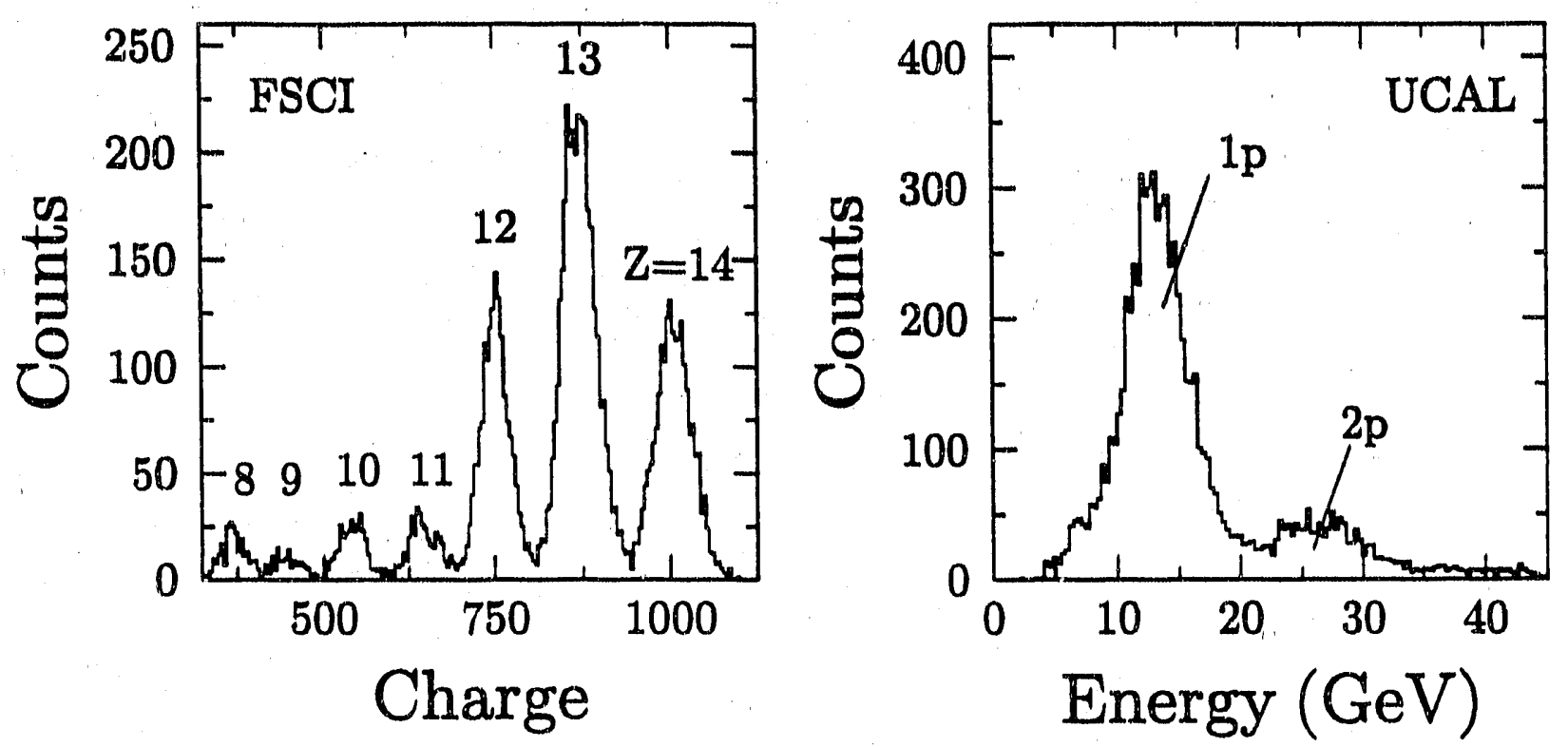

Figure 3: (a) Charge distribution for heavy ions and (b) calorimeter energy distribution for protons in peripheral data.

With these tools, we analyze raw data tapes and extract candidates for nuclear and peripheral events for further analysis and record them in a secondary set of $8 \mathrm{~mm}$ tapes. This procedure reduced our data sample by a factor of 4 , mostly due to the rejection of events containing downstream interactions. These tapes were used at this stage to study cross sections for channels with low-multiplicity final states, such as $1 \mathrm{p}+{ }^{27} \mathrm{Al}, 1 \mathrm{n}+{ }^{28} \mathrm{Si}$, and $1 p+1 n+{ }^{26} \mathrm{Al}$. The more complex peripheral channels are contaminated and information from the tracking chambers is required to reduce the background.

The E814 tracking chambers (described in detail elsewhere[8, 9]) have been used previously to study events produced via central interactions, in which only minimum ionizing particles are present. One of the problem we faced was to utilize these chambers in peripheral interactions, in which a highly charged ion is present in the final state. The heavy-fragment tends to produce more delta rays and other noise problems in the chambers making it difficult to obtain a good tracking resolution with the tracking software developed for minimum ionizing particles. 

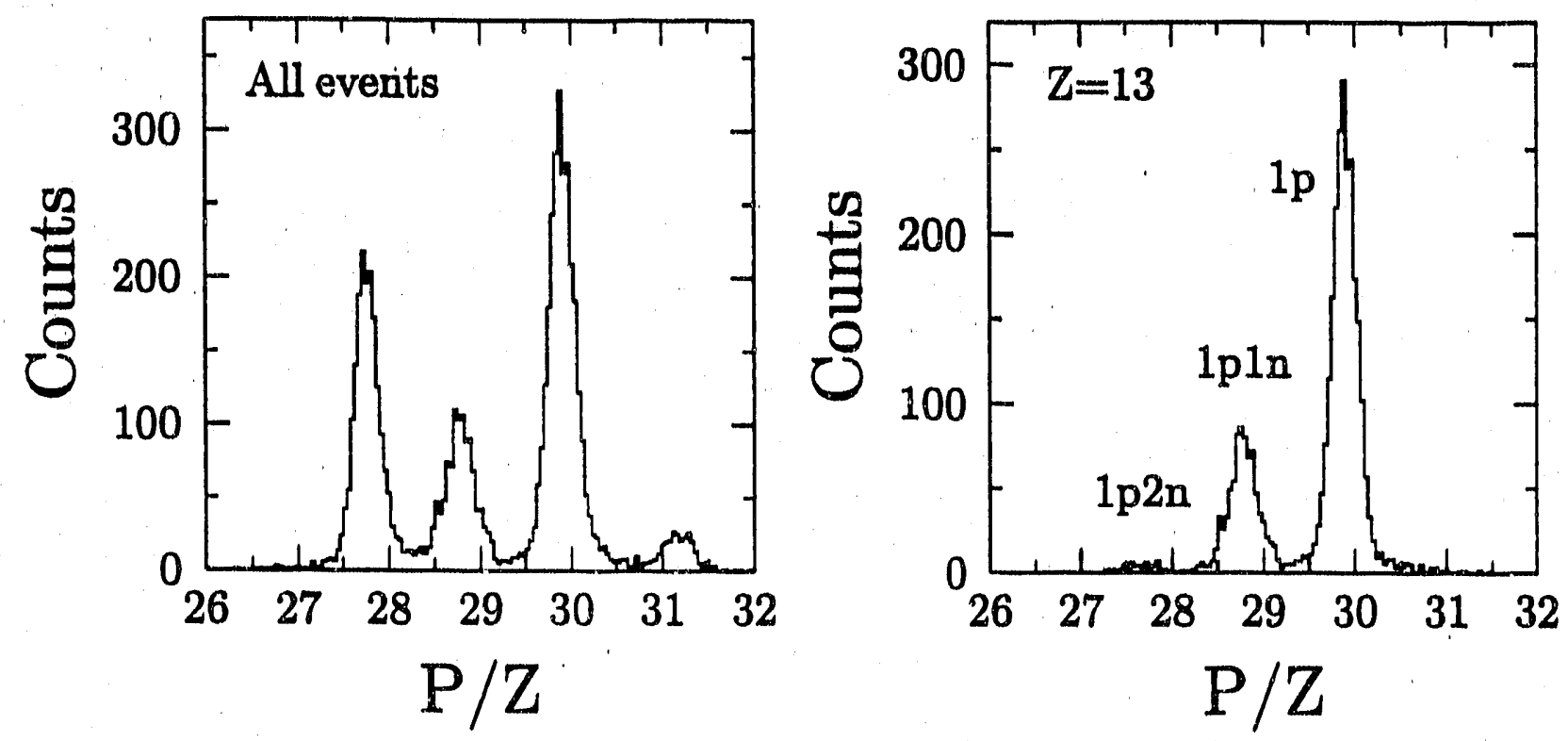

Figure 4: (a) Magnetic rigidity (P/Z) for the heavy ion track for all values of $\mathrm{Z}$; (b) same for $\mathrm{Z}=13$.

We worked extensively to understand these chambers in an heavy-ion environment and developed a pattern recognition program tailored to the peripheral analysis. The analysis was done in two parts. In the first part all tracks which deposit a correct charge in the pad sections of DCI, DCII and DCIII and also in the drift sections of DCII and DCIII are identified. Good tracks are identified with the possible hits in the upstream proton calorimeters by performing a crude pattern recognition. In the second stage, tracks were reconstructed with our geometrical/kinematic reconstruction program (GRIST). We have achieved a single cell resolution in the order of $300 \mu \mathrm{m}$ for both DCII and DCIII, for protons as well as heavy ions in the horizontal direction (see Appendix A). In the vertical direction we achieved $2.5 \mathrm{~mm}$ in DCII and $5 \mathrm{~mm}$ in DCIII. In the case of DCI, we achieved a resolution of about $200 \mu \mathrm{m}$ in the horizontal direction and $2 \mathrm{~mm}$ (wire separation) in the vertical direction. The work we performed on the beam vertex detector (see our previous DOE report) is an important part of the reconstruction, as it provies a precise measurement of the incident angle and position of the beam at the target.

In Figure 4 (a) we show the measured magnetic rigidity $(\mathrm{P} / \mathrm{Z})$ for heavy-ions for typical 
peripheral events after using our event reconstruction. With this information we are able to easily identify multinucleon emission channels. As an example, we show in Figure 4 (b) the magnetic rigidity for the events with measured $\mathrm{Z}=13$. Different peaks correspond to different isotopes and we can clearly identify $1 \mathrm{p}, 1 \mathrm{p} 1 \mathrm{n}$ and $1 \mathrm{p} 2 \mathrm{n}$ events.
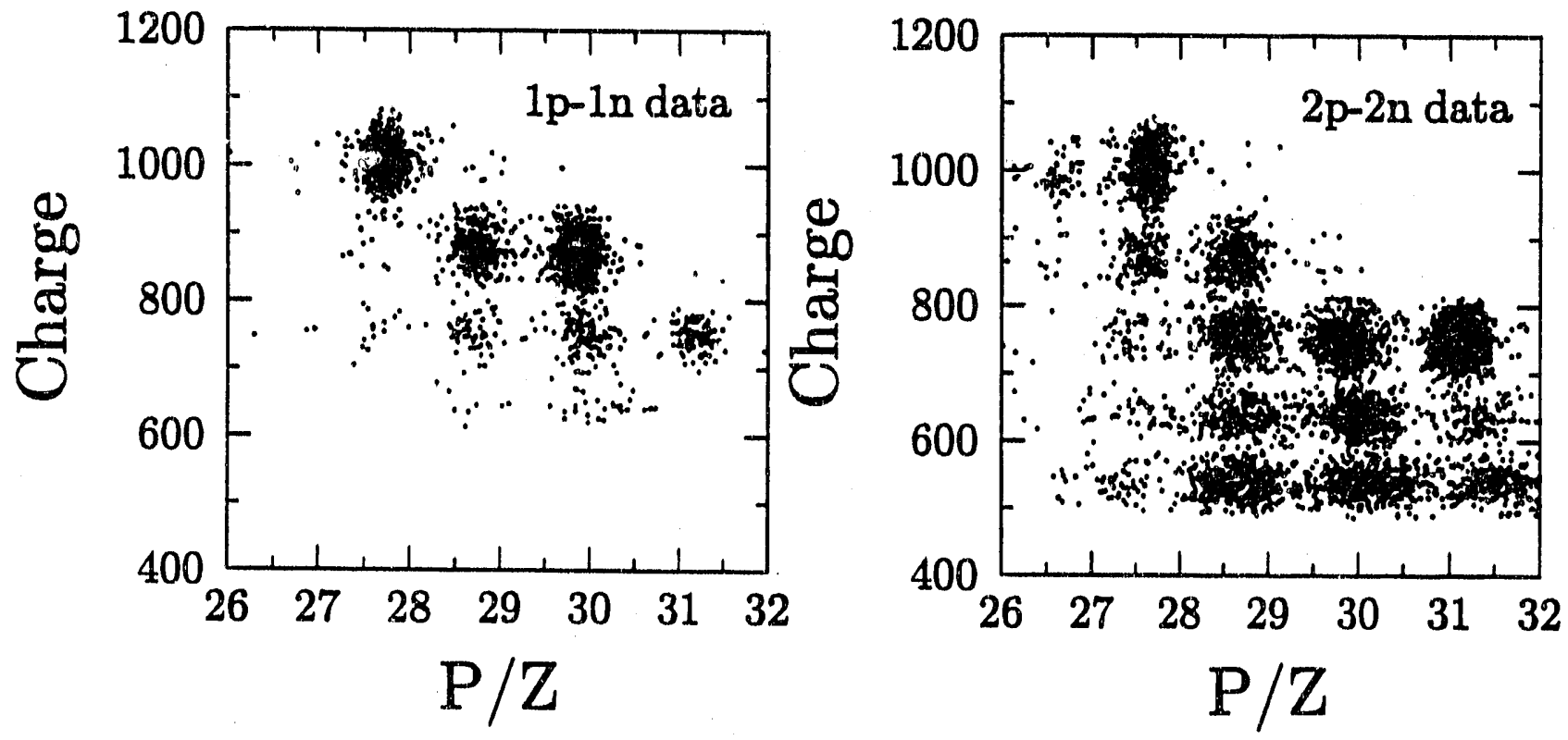

Figure 5: Two dimensional plots of the heavy ion charge (on a scale where $Z=14$ corresponds to 1000 ) vs its magnetic rigidity $(\mathrm{P} / \mathrm{Z})$. Each "island" corresponds to a different final state. (a) 1p triggers; (b) $2 p$ triggers.

In Figure 5, (a) and (b) we show the measured charge against the magnetic rigidity of the heavy-ion for a collection of events extracted through $1 \mathrm{p}-1 \mathrm{n}$ tapes and $2 \mathrm{p}-2 \mathrm{n}$ tapes. (Figure 4 are two projections of this plot.) We can identify a large number of peripheral channels from these and we have a good possibility of extracting rare channels such as $3 p+{ }^{25} \mathrm{Na}, 3 \mathrm{p}+1 \mathrm{n}+{ }^{24} \mathrm{Na}, 2 \mathrm{p}+1 \mathrm{~d}+{ }^{24} \mathrm{Na}, 4 \mathrm{p}+2 \mathrm{n}+{ }^{22} \mathrm{Ne}, 2 \mathrm{p}+1 \alpha+{ }^{22} \mathrm{Ne}$ etc. We are currently investigating the possibilities of extraction of some of these channels for full event reconstruction. 


\subsection{Physics Analysis}

One of the main goals of the E814 peripheral program was to measure the excitation energy of the ${ }^{28} \mathrm{Si}$ for possible channels and to get an insight into the Giant Dipole resonance. This can shed light into the Weiszäcker-Williams (WW) method and also to the possible multiphoton excitations. The E814 apparatus allows us to fully reconstruct peripheral channels with 2 kinematic constraints in order to study in detail such effects.

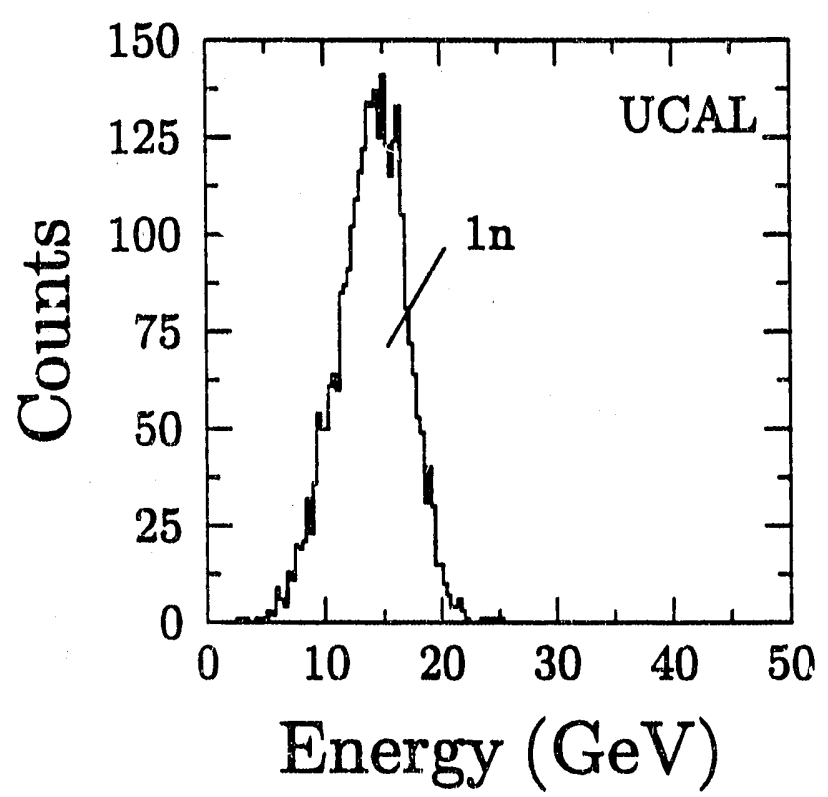

Figure 6: Neutron calorimeter energy distribution for peripheral events.

We began this work with the simplest decay channel $1 \mathrm{p}+{ }^{27} \mathrm{Al}$. This channel, apart from physics understanding, provides an excellent tool to understand the systematic problems associated with the detectors and resolution measurements. We also developed a simulation program which simulates the $1 p$ decay. It takes the known low energy photo nurlear cross section data for the ${ }^{28} \mathrm{Si}(\gamma, \mathrm{p})^{27} \mathrm{Al}$ channel and convolutes it with the WW photon spectrum and gives an excellent representation for our data. The preliminary result of this $1 \mathrm{p}$ analysis was presented at the April 1991 APS meeting. The work for $1 \mathrm{p}$ is now completed, and the results were submitted for publication in Physical Review C. A copy of this paper is included in this report as Appendix A. 
We are now working on a somewhat more difficult channel, $1 \mathrm{n}+{ }^{27} \mathrm{Si}$. This channel is interesting for many reasons. In literature, very limited data is available for low energy measurements because of the difficulty in measuring and detecting the neutron. This channel can also provide an understanding of how well calorimetry works in the reconstruction of exclusive events containing final state neutrons, and could lay the groundwork for studies of events with final state multineutron clusters like ${ }^{2} n$ or ${ }^{4} n$.
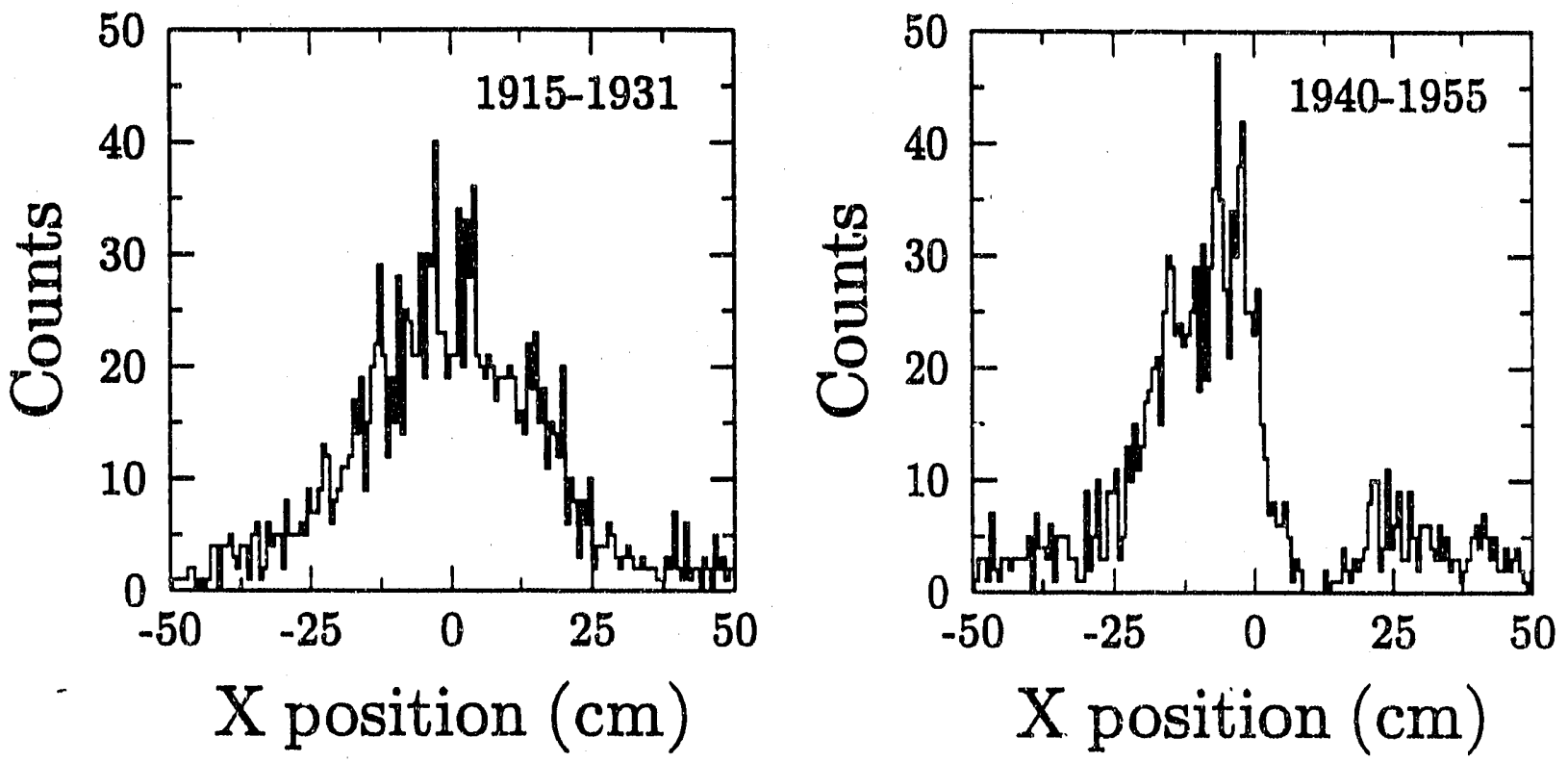

Figure 7: Horizontal position distribution for 1n data. (a) Portion of data sample with full calorimeter trigger; (b) portion of sample with one calorimeter module missing from trigger.

In Figure 6, we show the measured energy in the neutron calorimeters for the $1 n$ events. The efficiency of the neutron calorimeters are about $96 \%$. The low energy tail corresponds to neutrons with late showers which deposit very little energy in the calorimeter. In Figure, 7 (a) and (b) we show the measured $x$ positions at these calorimeters for the early part of the run and later part of the run. Due to a bad cable from one of the calorimeters (between $0-21 \mathrm{~cm}$ ) we have lost the contribution of one of the calorimeter modules to the trigger sum during the second part of the run which results in a gap in the position distributions. Fortunately, about half of the data does not suffer from this problem. Our calorimeter algorithms provide about $\approx 2 \mathrm{~cm}$ resolution in $\mathrm{x}$ and a little worse resolution 
in the $y$ direction.

Figure 8 shows the measured cross sections for in data (white circles) together with 1pln (black circles). The fit is a two component fit described in the 1p paper. Both of these show roughly a $\mathrm{Z}^{1.8}$ behavior with the target charge for the EMD part although the fit is shown only on In data. 1pln data suffered due to low statistics. The total cross section shows a non-negligible contribution from the "soft" nuclear part, specially for low $\mathrm{Z}$ targets.

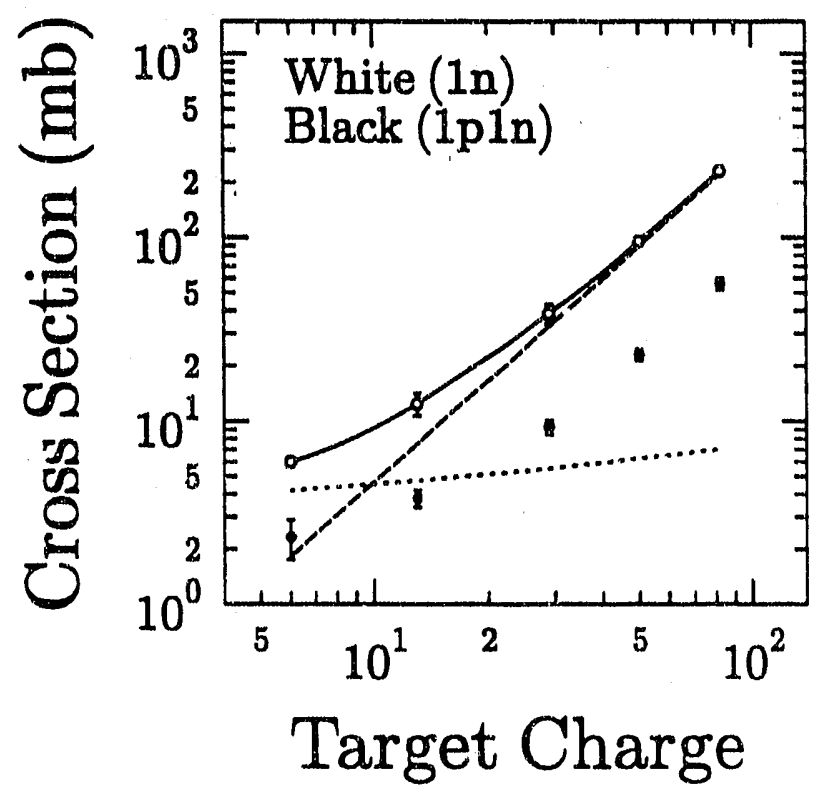

Figure 8: Cross section vs. target charge for $1 \mathrm{n}$ (white circles) and 1n $1 \mathrm{p}$ (black circles) data. The lines show the electromagnetic (dashed), nuclear (dotted), and total (solid) composition for the in data.

We carry out the same kinematic reconstruction process for the ln data sample as was used for the $1 \mathrm{p}$ sample, although we expect considerably worse resolution due to the lack of magnetic tracking for the neutron. This is easily seen in Figure 9, where we show the reconstructed momentum spectra for the $1 \mathrm{p}$ and $1 \mathrm{n}$ events. Note that the energy distribution in the $1 \mathrm{n}$ case is not quite symmetric, which we believe is a property of the calorimeter, arising from cases in which the neutron shower starts deep in the calorimeter. One of the challenges in this analysis, which is still in progress, will be to correctly handle 
the calorimeter response in the kinematic reconstruction. One of the tools at our disposal is the reconstructed angular distribution of the polar decay angle in the center-of-mass frame $\left(\theta^{*}\right)$.
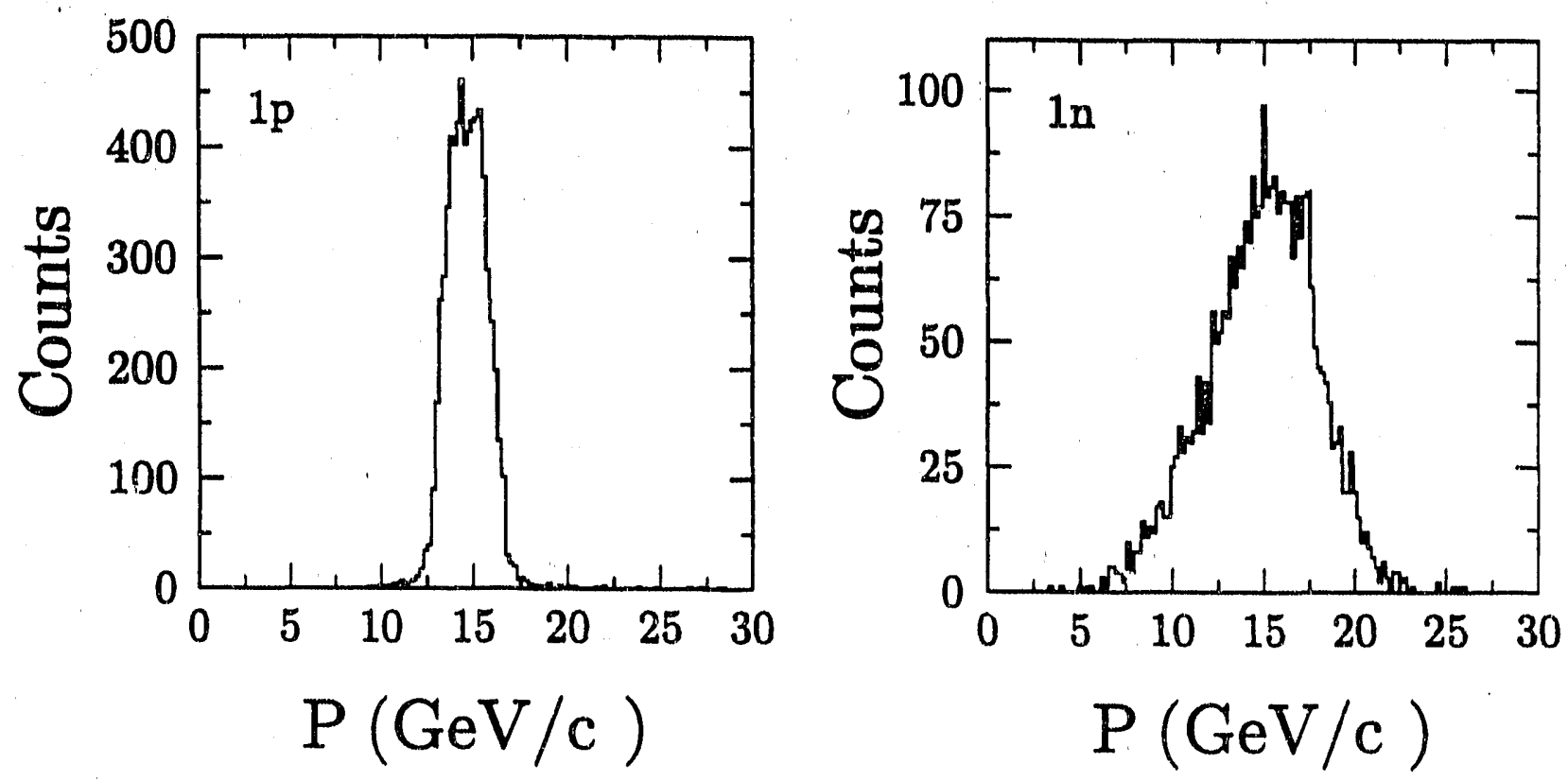

Figure 9: Momentum distributions from kinematic fit for (a) protons in $1 p$ events and (b) neutrons in $1 \mathrm{n}$ events. The resolution for the $1 \mathrm{n}$ data is considerably worse than that for the $1 \mathrm{p}$ events due to the use of the calorimeter rather than magnetic tracking for energy determination.

In Figure 10a we show the angular distribution found for the $1 \mathrm{p}$ events, which is consistent with isotropy, and in Figure 10b we show the corresponding quantity for the $1 \mathrm{n}$ events, which is clearly very different. The severe anisotropy in this case arises, we believe, from an improperly weighted measurement of the neutron energy, which, in the current analysis is assumed to be gaussian in nature. (Too low or too high energy measurements in UCAL tends to put events forward or backward in the CM system, artificially giving this shape.) We plan to develop a liklihood technique which will permit the introduction of a nongaussian response function for the calorimeter, and monitor the effect on the angular distribution, in which we will assume the same isotropy as has been measured in the $1 \mathrm{p}$ case.

Figure 11, (a) and (b) compare the excitation energy distribution for $1 \mathrm{p}$ and $1 \mathrm{n}$. The 

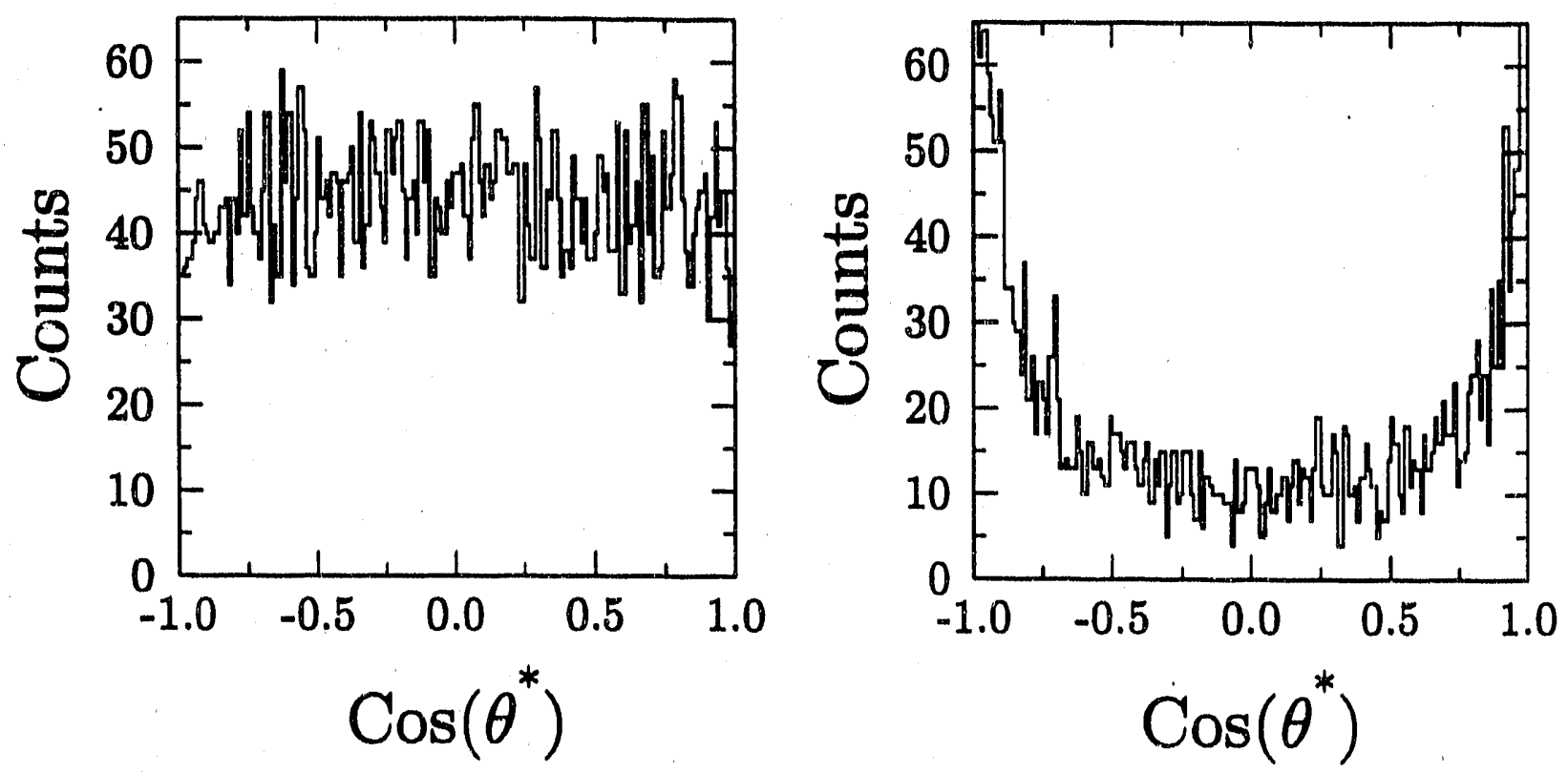

Figure 10: Distribution in $\cos \left(\theta^{*}\right)$ for (a) $1 \mathrm{p}$ and (b) $1 \mathrm{n}$ events. This quantity is very sensitive to errors in the energy determination. The lack of uniformity in the 1 n case probably arises from the non-gaussian behaviour of the calorimeter response.

kinematic thresholds for these events are $11.59 \mathrm{MeV}$ and $17.18 \mathrm{MeV}$ respectively. Clearly the neutron energy and position measurement also affects the excitation energy for the event. We plan to present preliminary results for this channel at the April 1992 APS meeting.

2.4 Studies of $2 p$ emission in the EMD of ${ }^{28} \mathrm{Si}$ and reconstruction of the invariant mass for the $2 \mathrm{p} 0 \mathrm{n}$ decay channel

Following the encouraging results obtained in the reconstruction of the invariant masses in the dissociation of ${ }^{28} \mathrm{Si}$ by emission of one proton, we are also pursuing a study of EMD into the $2 p$ emission channels, particularly the $2 \mathrm{p} 0 \mathrm{n}$ decay channel. These decay modes require larger photon energies to be produced, and might reveal new aspects in the excitation process of ${ }^{28} \mathrm{Si}$. Also, the event ceconstruction of the two nucleon emission channel, $2 \mathrm{p} 0 \mathrm{n}$, opens up the possibility for looking for correlated $2 \mathrm{p}$ emission if this can happen in peripheral collisions in the energy region spanned by our data. 

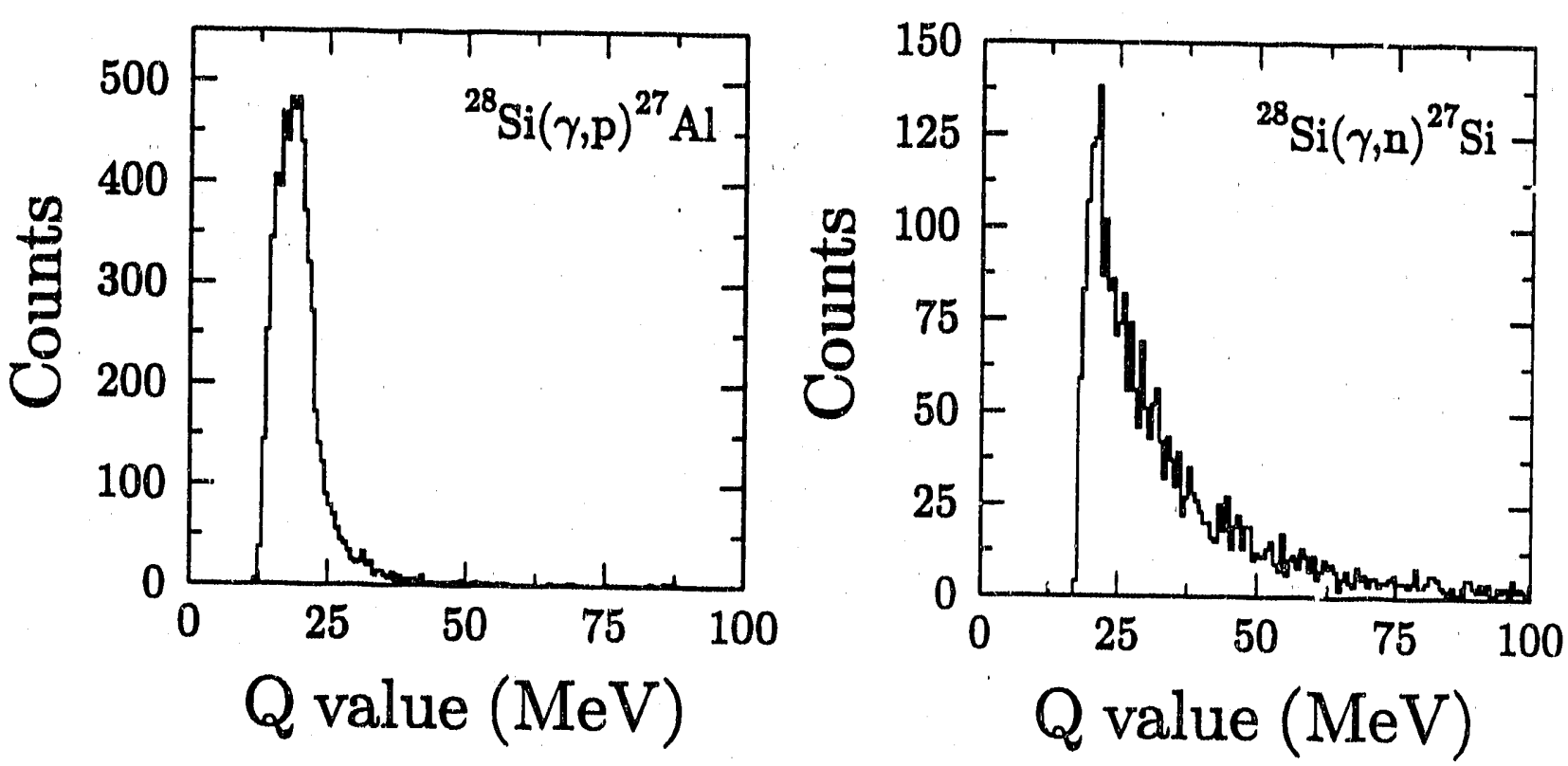

Figure 11: Excitation energy distribution for (a) $1 \mathrm{p}$ and (b) In events. The lower resolution for the neutron position and energy causes a lower resolution in the excitation energy determination.

\subsubsection{Event Selection}

To select good "EM events" (events from the EMD of ${ }^{28} \mathrm{Si}$ ) we use the same method described in detail in refs. [3] and [6]. To select good 2pxn events we impose cuts on the charge and energy of the heavy ion $(\mathrm{Mg})$ as well as of the protons, measured in the corresponding forward scintillators and calorimeters (details of the analysis procedure are described in ref. [6]), and we also demand appropriate energy in the neutron calorimeters. To further improve the quality of the selection procedure, we require the magnetic rigidity $\mathrm{P} / \mathrm{Z}$ of the heavy ion (see Figure 4) to be within certain limits. This step is needed since inefficiency and poor energy resolution in the neutron calorimeters can allow background (primarily $2 p(x+1) n$ ) events to be present in the $2 p x n$ sample. Combined with the charge information, this analysis permits us to identify the mass number of the heavy ion, thus enabling the extraction of a cleaner sample of $2 \mathrm{pxn}$ events.

To perform the heavy ion $\mathrm{P} / \mathrm{Z}$ analysis we use information provided by the drift chambers of the E814 forward spectrometer, and we reconstruct the heavy ion track. 


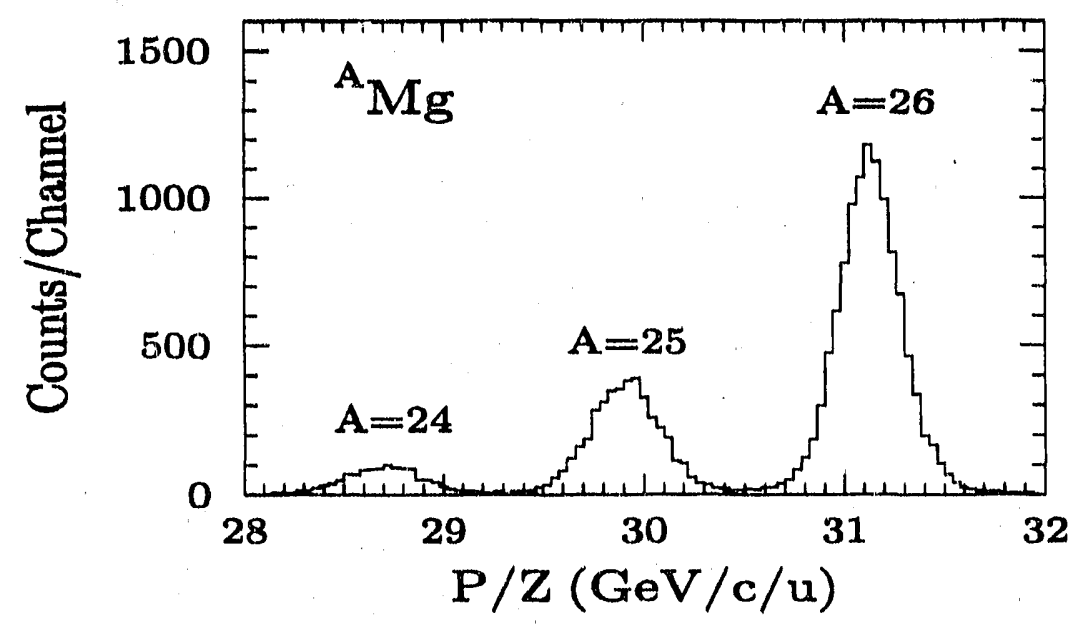

Figure 12: Momentum distribution of $\mathrm{Mg}$ isotopes corresponding to 2pxn emission channels from the decay of ${ }^{28} \mathrm{Si}^{*}$.

Figure 12 shows the $\mathrm{P} / \mathrm{Z}$ distribution for events where the heavy ion has charge number $\mathrm{Z}=12$. Indicated in the figure are the mass numbers corresponding to the various $\mathrm{Mg}$ jsotopes, and we note the clean separation between the different groups.

This method of mass identification of the heavy ion is currently being applied to many other studies of the E814 peripheral collision data, such as studies of fragmentation processes, EMD into multi-nucleon emission channels and search for rarer decay modes, involving for example the emission of $\alpha$ particles. It is also used in the selection of specific samples of events for studies involving invariant mass reconstruction, which are presently being done for the $2 \mathrm{p} 0 \mathrm{n}$ and $1 \mathrm{n}$ decay channels.

\subsubsection{Cross Sections Studies}

Preliminary cross sections for the EMD of ${ }^{28} \mathrm{Si}$ into $2 \mathrm{p} 0 \mathrm{n}, 2 \mathrm{p} 1 \mathrm{n}$ and $2 \mathrm{p} 2 \mathrm{n}$ decay channels were extracted for targets of $\mathrm{Pb}, \mathrm{Sn}, \mathrm{Cu}$ and $\mathrm{Al}$. Figure 13 shows the cross sections as a function of the target $Z$. Under the assumption that the Coulomb and the nuclear parts do not interfere, and that the nuclear component scales as the sum of the target 


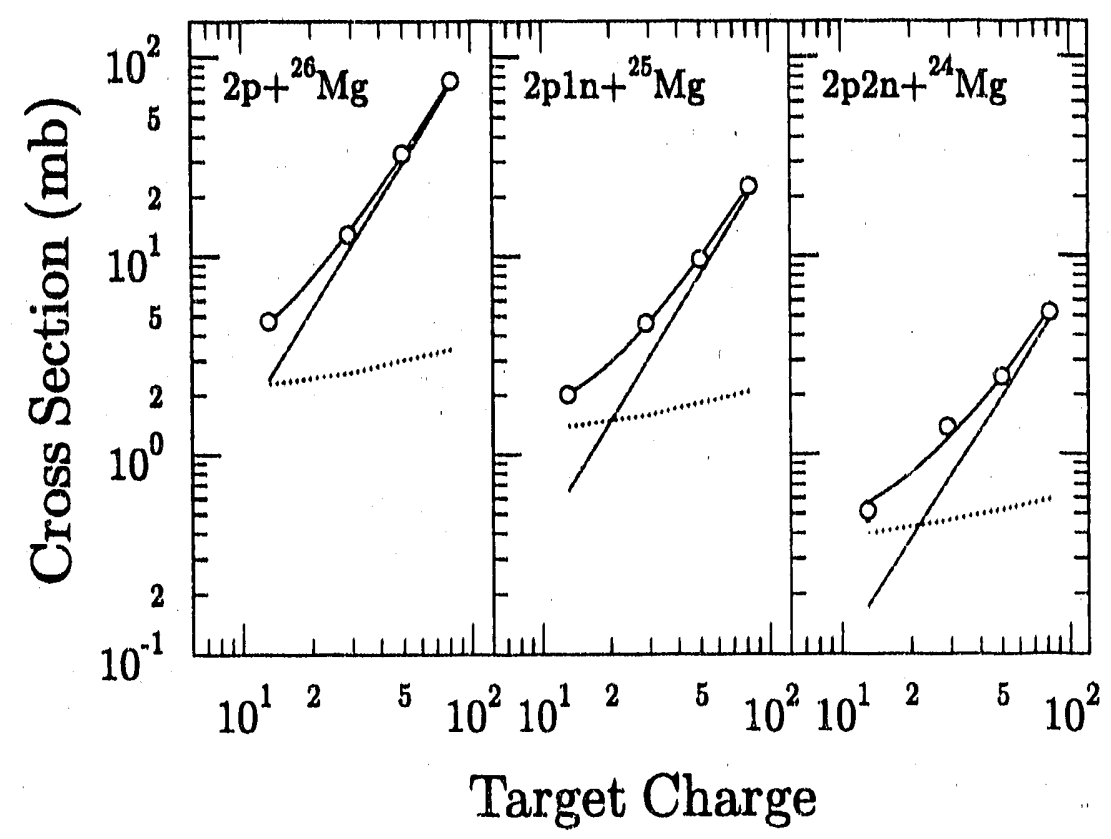

Figure 13: Cross sections for the $2 \mathrm{p} 0 \mathrm{n}, 2 \mathrm{p} 1 \mathrm{n}$ and $2 \mathrm{p} 2 \mathrm{n}$ decay channels from ${ }^{28} \mathrm{Si}^{*}$ as a function of the target $\mathrm{Z}$.

and projectile perimeters, the electromagnetic component scales approximately as $\mathrm{Z}^{1.8}$, in agreement with other EMD studies [11]. In the figure, the dashed curve represents the EM component, the dotted curve is for the nuclear component, and the solid curve is for the sum of the two components.

\subsubsection{Invariant mass reconstruction studies for the $2 \mathrm{p} 0 \mathrm{n}$ decay channel}

To handle the kinematic reconstruction of the 3-body decay channel, we found it convenient to consider it as a composite 2-body decay system. We emphasize that this is not a physical assumption, and that we can always convert the quantities extracted in the frame of any 2-body decay frame to the original frame of the decaying projectile by doing the appropriate Lorentz transformations.

We consider the following decay scheme: 
- ${ }^{28} \mathrm{Si} \rightarrow{ }^{26} \mathrm{Mg}+\mathcal{D}$ ("diproton")

- $\mathcal{D} \rightarrow \mathrm{p}+\mathrm{p}$

We define the following quantities:

- $\mathrm{Ex}_{1}$ is the excitation energy of the excited ${ }^{28} \mathrm{Si}^{*}$.

- $\mathrm{Q}_{2}$ is the mass of the diproton less the rest masses of the two protons.

- $\theta^{*}$ is the polar angle of the ${ }^{26} \mathrm{Mg}$ momentum vector with respect to the beam momentum vector in the rest frame of the ${ }^{28} \mathrm{Si}$ projectile.

- $\phi^{*}$ is the azimuthal angle of the decay plane about the beam momentum vector, in the first 2-body decay subsystem, referred to the horizontal plane.

- $\theta_{p}$ is the polar angle of the momentum vector of the proton going forward with respect to the momentum vector of the diproton in the rest frame of the diproton.

- $\phi_{p}$ is the azimuthal angle of the decay plane about the diproton momentum vector, in the diproton frame, referred to the decay plane of the first 2-body decay subsystem.

The basic steps for event reconstruction are described in detail in ref. [3]. However a number of extra procedures are introduced to handle the reconstruction of the two proton tracks, since they lie close together in a considerable fraction of the events. The reconstructed mass of the diproton shows a distribution concentrated at very low values, as is shown below, and under these circumstances the momentum vectors of the two protons are almost identical, so they follow practically the same trajectory.

The main new features introduced in the reconstruction program are:

- A better definition of the "roads" defined by the pads in DC2 and DC3 to take into account regions of different pad widths in each chamber. These "roads" are used to perform an initial selection of the TDC hits belonging to each track. 
- Inclusion of a second alternative for creating the "roads" corresponding to each track by swapping, in one of the chambers, the pad information initially assigned to each track.

- For the cases where the two proton tracks cross the same pad in one of the chambers, inclusion of a second alternative for fitting the track through the TDC hits by swapping, in the chamber having the common pad, the set of TDC hits initially associated to each proton track. Studies done with simulated events showed that improper assignment of the set of TDC hits to each track was not unlikely to occur, resulting in wrong values for the reconstructed quantities.

- Inclusion of special criteria to choose the correct alternative for the proton hits, which consists of reconstructing the tracks for both configurations, going through the magnets up to the target, and making the choice based on (a) how mainy hits have been found in $\mathrm{DC1}$, (b) how many TDC hits are found to lie on each track, and (c) the combined $\chi^{2}$ values of the fit to the two tracks. Tests done with simulated events show that the method works well.

By using simulated events we also determine the resolution attainable in the reconstruction of invariant masses and decay angles. Basically, we generate the positions of the tracks in all the detection planes of our apparatus, in which we include distortions due to multiple scattering in the target and in the raterials present in the path of the particles, and resolution of the detectors. The set of events is then reconstructed and we compare the results from the reconstruction to the quantities used to produce the event. Figure 14 shows the differences between these two quantities for: $(a)$ the excitation energy of the ${ }^{28} \mathrm{Si}^{*} ;(b)$ the mass of the diproton; $(c)$ the polar angle $\theta^{*} ;(d)$ the azimuthal angle $\phi^{*} ;$ (e) the polar angle $\theta_{p i}(f)$ the azimuthal angle $\phi_{p}$. The distributions shown correspond to the $\mathrm{Pb}$ target. Our resolution is about $1.7 \mathrm{MeV}$ in the excitation energy of the ${ }^{28} \mathrm{Si}^{*}$ and $0.5 \mathrm{MeV}$ in the mass of the diproton, which are remarkable when we consider that the beam energy is of hundreds of $\mathrm{GeV}$. The angular distributions also show good overall 

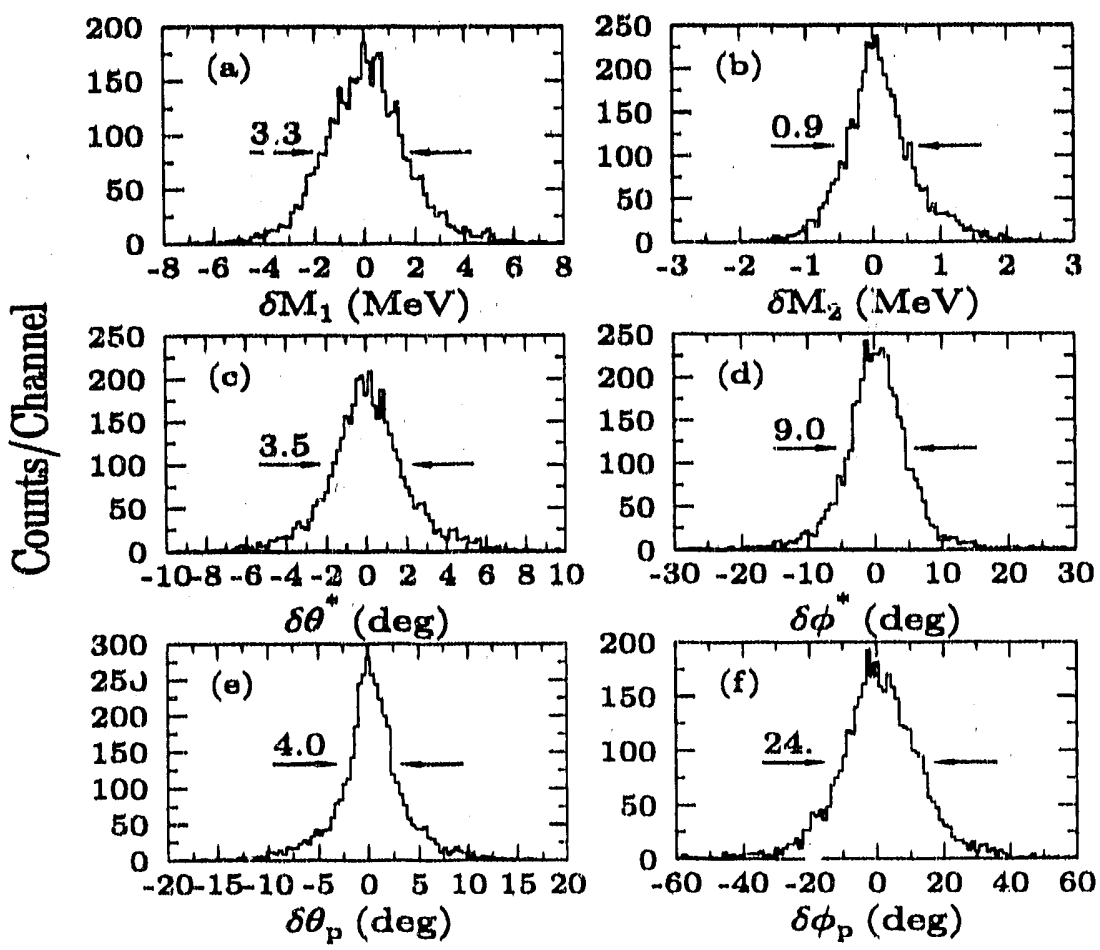

Figure 14: Distribution of the differences between reconstructed quantities and the ones used to produce the event.

resolution except for the azimuthal angle of the diproton decay, which is understandable if we remember that most of the time the two protons are quite close together.

Some preliminary reconstructed data are presented in Figs. 15 and 16. Figure 15 shows the distributions of $(a)$ the excitation energy of the ${ }^{28} \mathrm{Si}^{*}$ and $(b)$ the mass of the diproton system (less the rest masses). In Figure 16 we show the angular distributions of the decay. The sharp rise above the threshold that we see in Figure 15(a) gives an indication of our good resolution in excitation energy. If we compare this distribution with the one for the excitation energy of ${ }^{28} \mathrm{Si}^{*}$ in the $1 \mathrm{p}$ decay channel $\mathrm{n}$ the $1 \mathrm{p}$ decay channel (Figure 5 of ref. [3]), we note that in the present case of $2 p$ emission the high energy tail extends further out. In part $(b)$ of Figure 15 we see that the diproton mass distribution is peaked at very low values, which means that the two protons will have close trajectories, as noted 

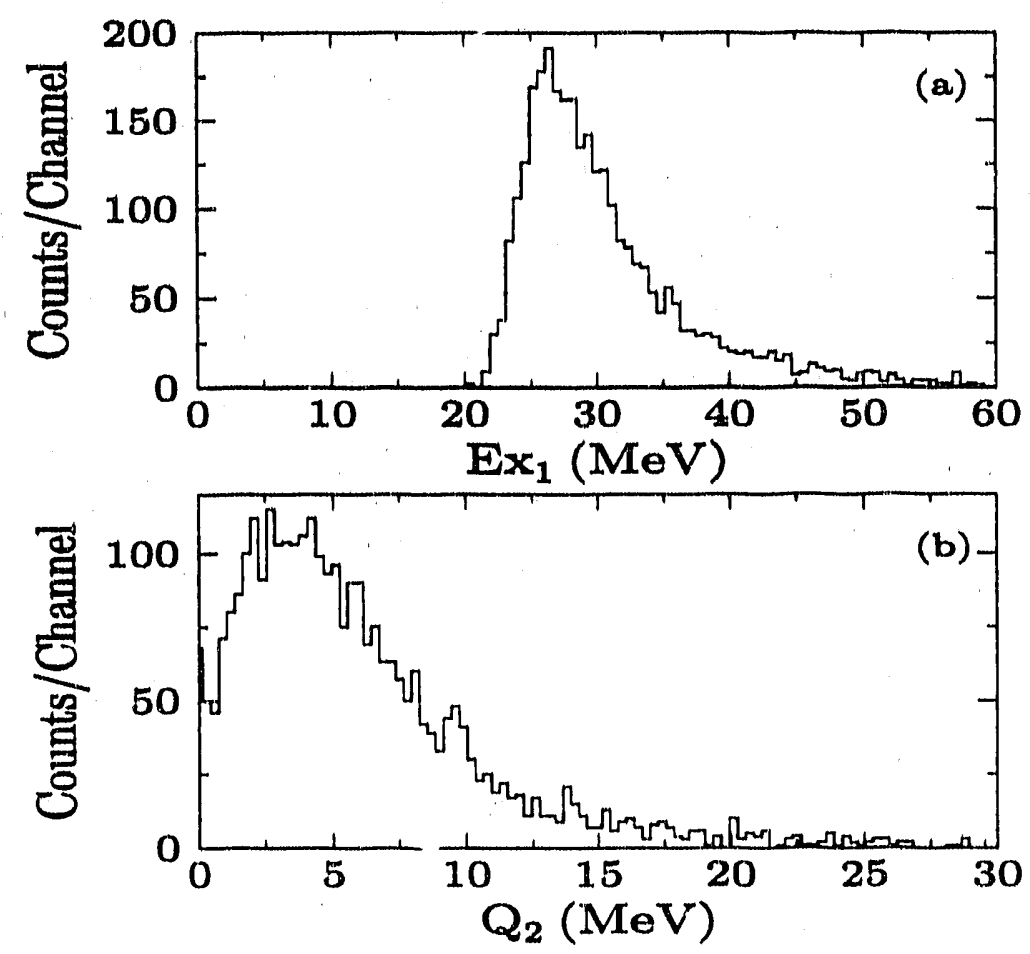

Figure 15: Experimental Distribution of $(a)$ excitation energy of ${ }^{28} \mathrm{Si}^{*} ;(b)$ mass of the diproton system (less the rest masses).

above.

The $\cos \left(\theta^{*}\right)$ distribution shown in Figure $16(a)$ indicates that apparently the ${ }^{26} \mathrm{Mg}$ is ernitted preferentially in the forward direction. However studies made using simulated events show that this behaviour could be due to misalignment of the detectors or imprecise information about the incident beam momentum. Studies of the incident heam momentum provided by the Gauss Clock (a pulse train provided by the AGS whose frequency is related to the instantaneous magnetic rigidity of the extracted beam) indicate that from one set of runs to another the beam rigidity $\mathrm{P} / \mathrm{Z}$ can vary by as much as 0.3 $\mathrm{GeV} / \mathrm{c} / \mathrm{u}$. On the other hand our studies based on simulation indicate that a systematic error of 0.2 in the beam $\mathrm{P} / \mathrm{Z}$ value already produce angular distributions with the $\cos \left(\theta^{*}\right)$ peaked at one of the extremes. This effect is caused by an error in the Lorentz factor, and 

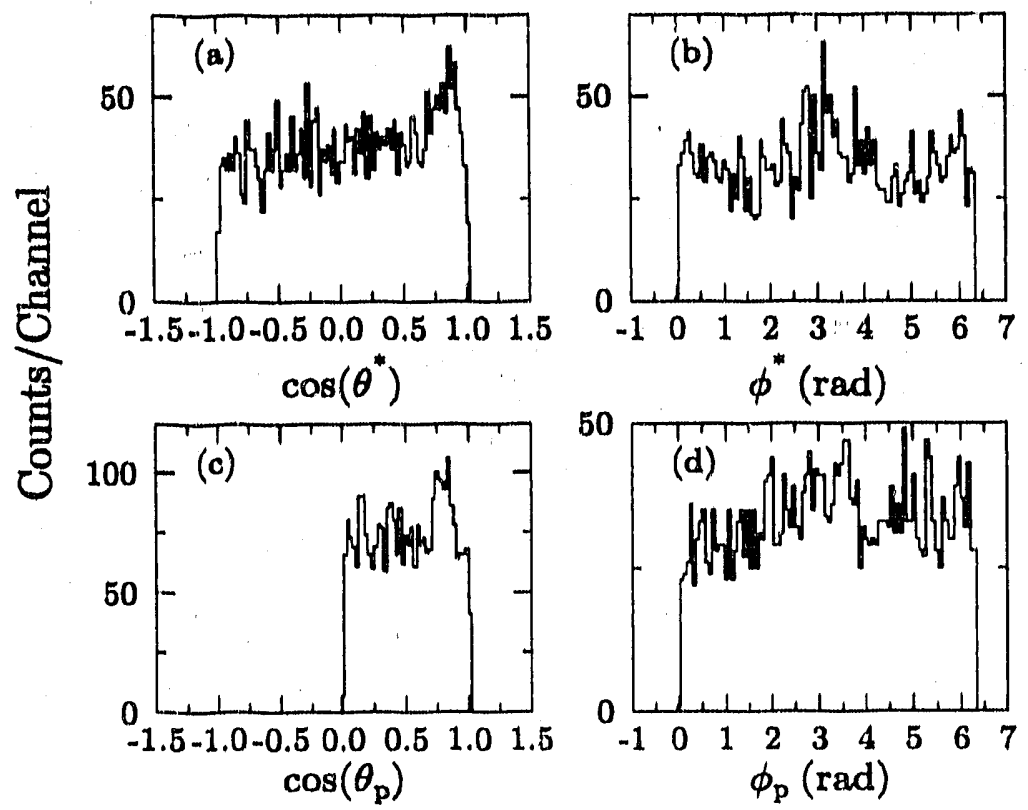

Figure 16: Experimental Angular Distributions in the composite 2-body decay of ${ }^{28} \mathrm{Si}^{*}$ into ${ }^{26} \mathrm{Mg}$ $+2 \mathrm{p}$.

it is therefore important to determine the absolute value of the beam momentum. We are able to verify from magnetic tracking that variations in the momentum are properly measured by the Gauss Clock. However additional work is required to ascertain whether there can be a small offset at the level of $0.1 \mathrm{GeV} / \mathrm{c}$. Studies of this effect are still in progress.

We plan to present preliminary results of the $2 \mathrm{p}$ channel reconstruction at the April 1992 meeting of the APS.

\section{Analysis of Central Collisions}

\subsection{Introduction}

Our group has been studying central collisions in two ways, through the charged multiplicity distributions as measured in the the silicon multiplicity detector and through the 
$E_{T}$ distributions measured in the participant calorimeter.

The charged multiplicity work was the $\mathrm{Ph}$.D. thesis of one of our graduates students, $\mathrm{M}$. K. Jayananda, and this research was completed during the past year. A copy of his thesis is included as Appendix B of this report. The topics covered included total multiplicity distributions for the three targets used in the central data-taking $(\mathrm{Al}, \mathrm{Cu}$, and $\mathrm{Pb})$, a study of $d N / d \eta$, including an evaluation of the negative binomial parameters, and a study of two-particle correlation functions. The techniques and conclusions are reported in detail in Appendix B. A paper on the charged multiplicity distributions measured in E814 has been submitted to Physical Review C [7].

The analysis of the $E_{T}$ distributions from the participant calorimeter is still underway. It is discussed in detail in the following section.

\subsection{Studies of $E_{T}$ with the Participant Calorimeter}

\subsubsection{Detector Description}

Table 2: List of the mechanical properties of the participant calorimeter

\begin{tabular}{|c|c|c|c|c|}
\hline & EM1 & EM2 & HAD1 & HAD2 \\
\hline Number of Pb plates & 5 & 5 & 20 & 19 \\
Thicknes of Pb plates & $1.0 \mathrm{~cm}$ & $1.0 \mathrm{~cm}$ & $1.0 \mathrm{~cm}$ & $1.0 \mathrm{~cm}$ \\
\hline Number of Fe plates & 1 & 1 & 1 & 1 \\
Thicknes of Fe plates & $1.6 \mathrm{~cm}$ & $1.6 \mathrm{~cm}$ & $1.6 \mathrm{~cm}$ & $1.6 \mathrm{~cm}$ \\
\hline Number of scint. layers & 6 & 6 & 24 & 23 \\
Number of scint. plates & 768 & 768 & 3072 & 3072 \\
Thicknes of scint. plates & $0.3 \mathrm{~cm}$ & $0.3 \mathrm{~cm}$ & $0.3 \mathrm{~cm}$ & $0.3 \mathrm{~cm}$ \\
Type of scint. plates & BC408 & BC408 & KSTI-430 & KSTI-430 \\
\hline Number of PMT's & 128 & 128 & 128 & 128 \\
\hline
\end{tabular}

The participant calorimeter is designed to be approximately azimuthally symmetric, 

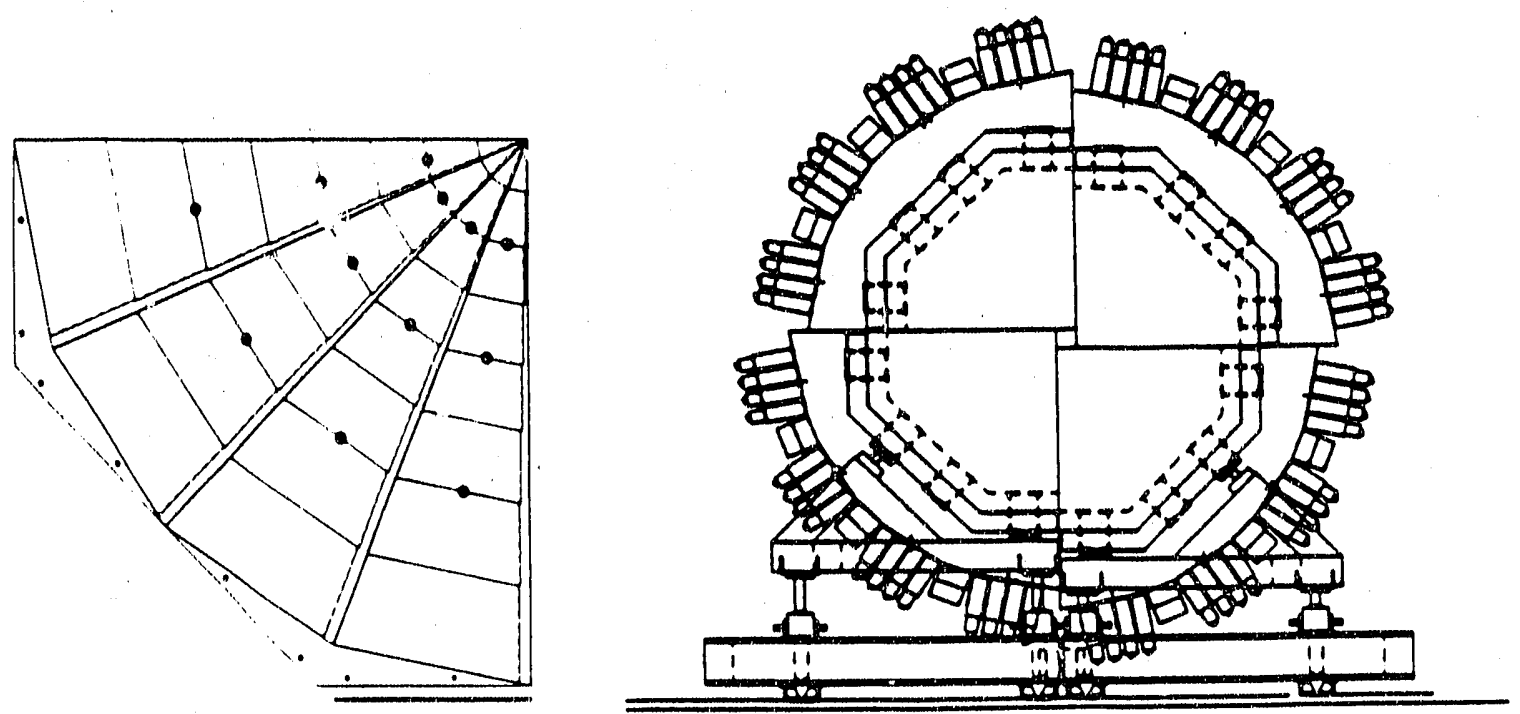

Figure 17: Participant calorimeter, seen by the beam. The left drawing details transverse segmentation in quatrant IV.

covering the polar angle $1.6^{\circ}<\theta<48.0^{\circ}$, corresponding to pseudorapitity region 0.81 $<n<4.3$. It uses lead plates as radiators and plastic scintillators coupled with optical fibers for sampling readouts. Every 6 th lead plate is substituted with an iron plate for mechanical stability.

The calorimeter has a depth of four interaction lengths and a radius of 33 inches. It is segmented tranversely into 8 radial and 16 azimuthal segments and longitudinally into 4 depth segments, and is read out with an array of 512 phototubes. A list of the mechanical properties is given in Table 2 and a drawing of the calorimeter is shown in Figure 17.

\subsubsection{SEB Test beam for Calibration}

The procedure to calibrate PCAL is that a group of towers accessible to the beam which lie in the horizontal azimuthal segment (pie) with different sizes and positioned at different values of pseudorapidity can be calibrated first with SEB beam, with the reasonable 
assumption that all towers at the same pseudorapidity receive the same amount of $\mathrm{E}_{T}$ the calibration is then spread around the detector according to the calibrated tower with similar pseudorapidity and same depth.

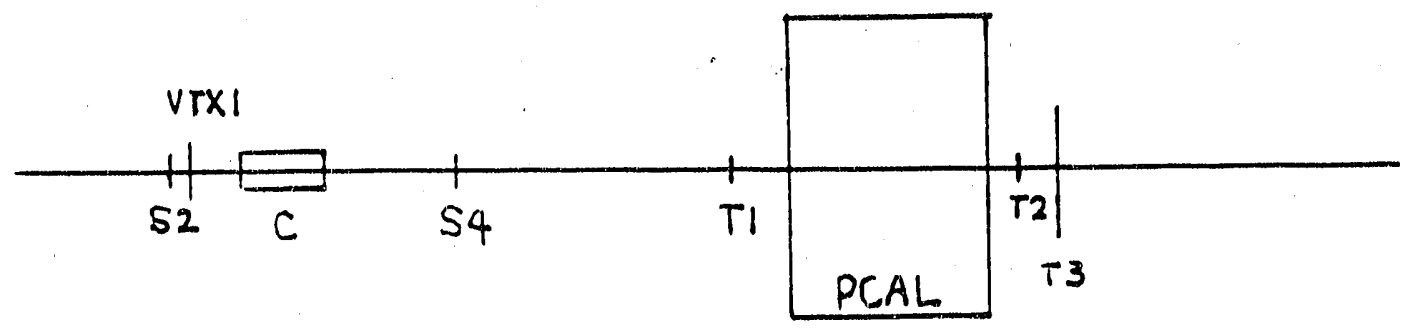

Figure 18: Setup used in the calibration of the participant calorimeter.

Table 3: Conditions for the calibration runs, showing the techniques employed for the particle identification

\begin{tabular}{|c|c|c|c|c|c|}
\hline momentum(Gev/c) & $\mu$ & e & $\pi$ & p & gas in Cherekov \\
\hline 1.0 & T1,T2 & C & no C & TOF & air(1.0atm) \\
2.0 & T1,T2 & C & no C & TOF(limit) & air(1.0atm) \\
-4.0 & T1,T2 & C & no C & - & air(1.0atm) \\
+4.0 & T1,T2 & no C & no C & C & freon(1.0atm) \\
10.0 & T1,T2 & no C & no C & C & freon(1.0atm) \\
14.6 & T1,T2 & no C & no C & C & freon(1.0atm) \\
\hline
\end{tabular}

The calibration was performed in April 1991, when AGS SEB proton beam was available to E814. The setup for beam calibration is shown in Figure 18. It consists of a vertex detector(VTX1), a pair of beam scintilator horoscopes(S2,S4), a Cherenkov counter(C), two $1.0 \mathrm{~cm}$ diameter $\mu$ scintilator counters $(\mathrm{T} 1, \mathrm{~T} 2)$ in both front and back of the PCAL, 
and a 12 inch paddle scintilator(T3) at the end. Two drift chambers were also turned on for beam momentum test. Table 3 shows the identifications of different particles at different momenta.

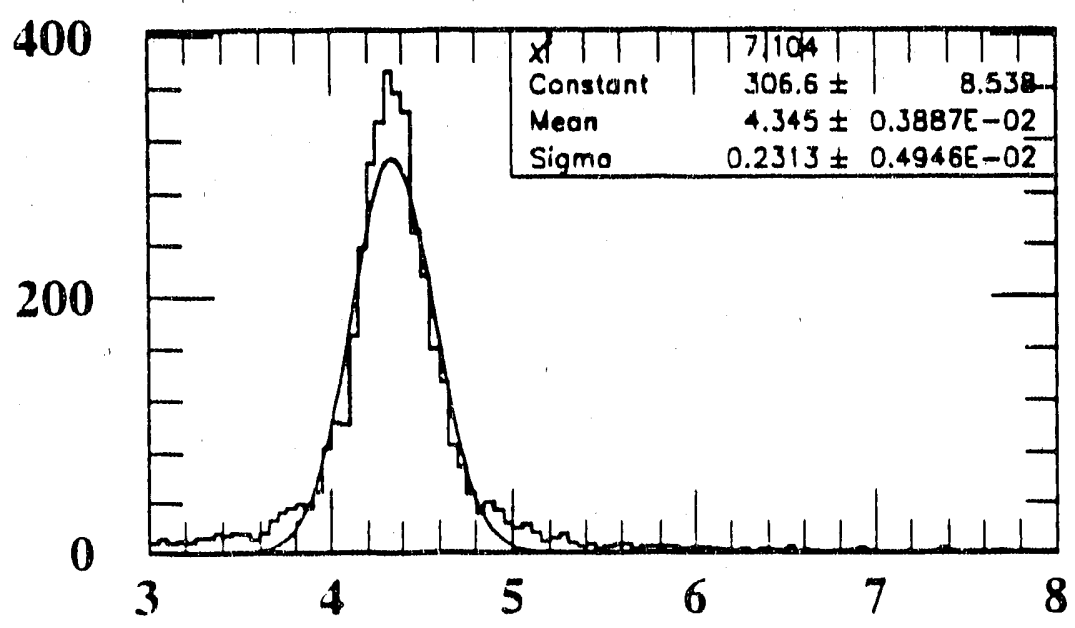

Figure 19: Momentum of the beam used in the PCAL calibration, obtained from tracking in the E814 magnetic spectrometer.

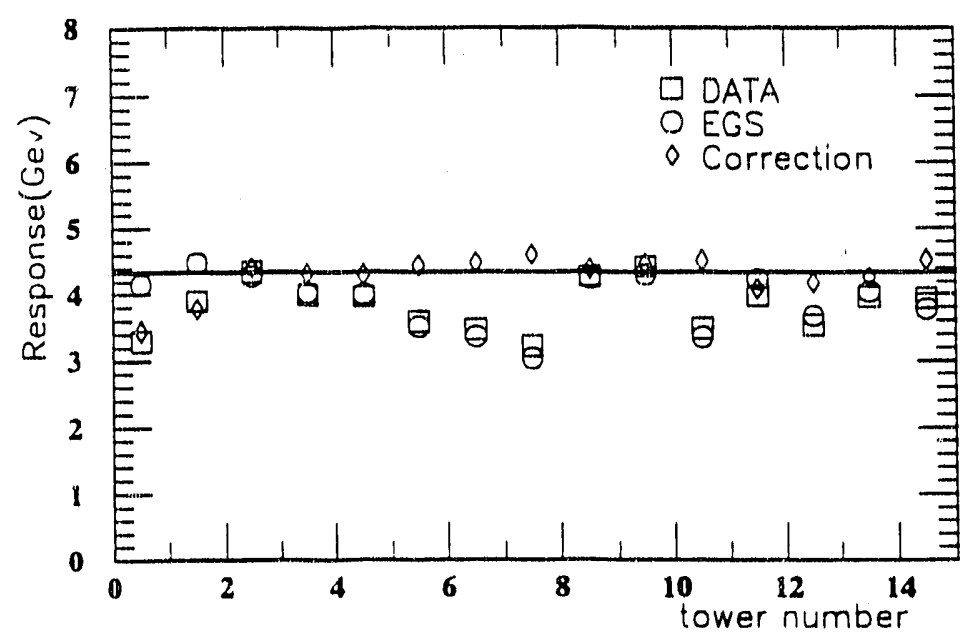

Figure 20: Mean values of the electron energy for a beam of $4.31 \mathrm{GeV} / \mathrm{c}$ (squares). Monte Carlo simulated data are shown as circles. Corrected data are shown as diamonds.

Figure 19 shows the beam momentum at a nominal value $4.31 \mathrm{Gev} / \mathrm{c}$, the histogram 


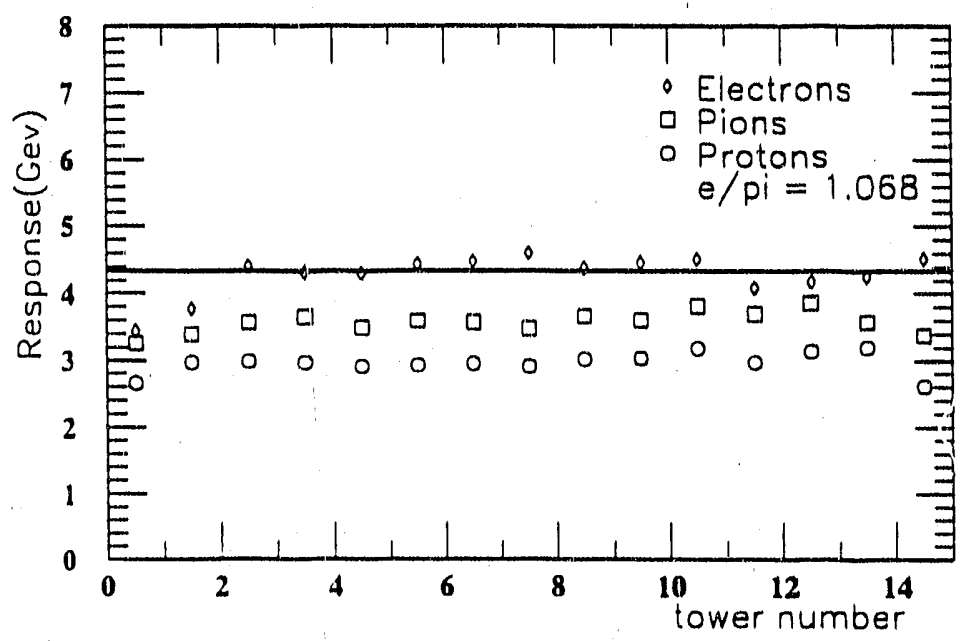

Figure 21: Comparison of electron, pion and proton responses for a beam of $4.31 \mathrm{GeV} / \mathrm{c}$. From these data we obtain a value of the $e / \pi$ ratio of 1.07 .

is the measured value using drift chambers and GRIST track reconstruction code. All towers in pie 16 and towers of the 2 smallest sizes in quadrant IV(shown in Figeure 17) were calibrated at this energy. For each tower, a tape of about 8,000 events were recorded with mixed trigger of $\mu$, electron, $\pi$ and proton.

Relative calibrations of the photomultinliers for these towers in the beam are achieved using $\mu$ data at $4.31 \mathrm{Gev} / \mathrm{c}$. The energy loss of $\mu$ 's while traversing the calorimeter is essentically minimum ionizing and can be calculated basing on the Bethe-Bloch formula to set the relative calibrations for all towers seen by the bearn.

Figure 20 shows electron energy depositions in all these calibrated towers, with relative gain matching from $\mu$ data. The large fluctuations are due to the fact that optical coupling varies drastically from plate to plate and from tower to tower. By putting in the coupling constants obtained from ${ }^{60} \mathrm{Co}$ source scan, EGS simulation tracks these fluctuations to within 5\%, except the first two towers because of the leakage into the PCAL center hole. Average electron response is then corrected by taking out the effect of optical coupling.

Pion and proton responses were also measured and Figure 21 shows them together with electrons. Taking the average of size $4,5,6$, where there is minimal side leakage, 

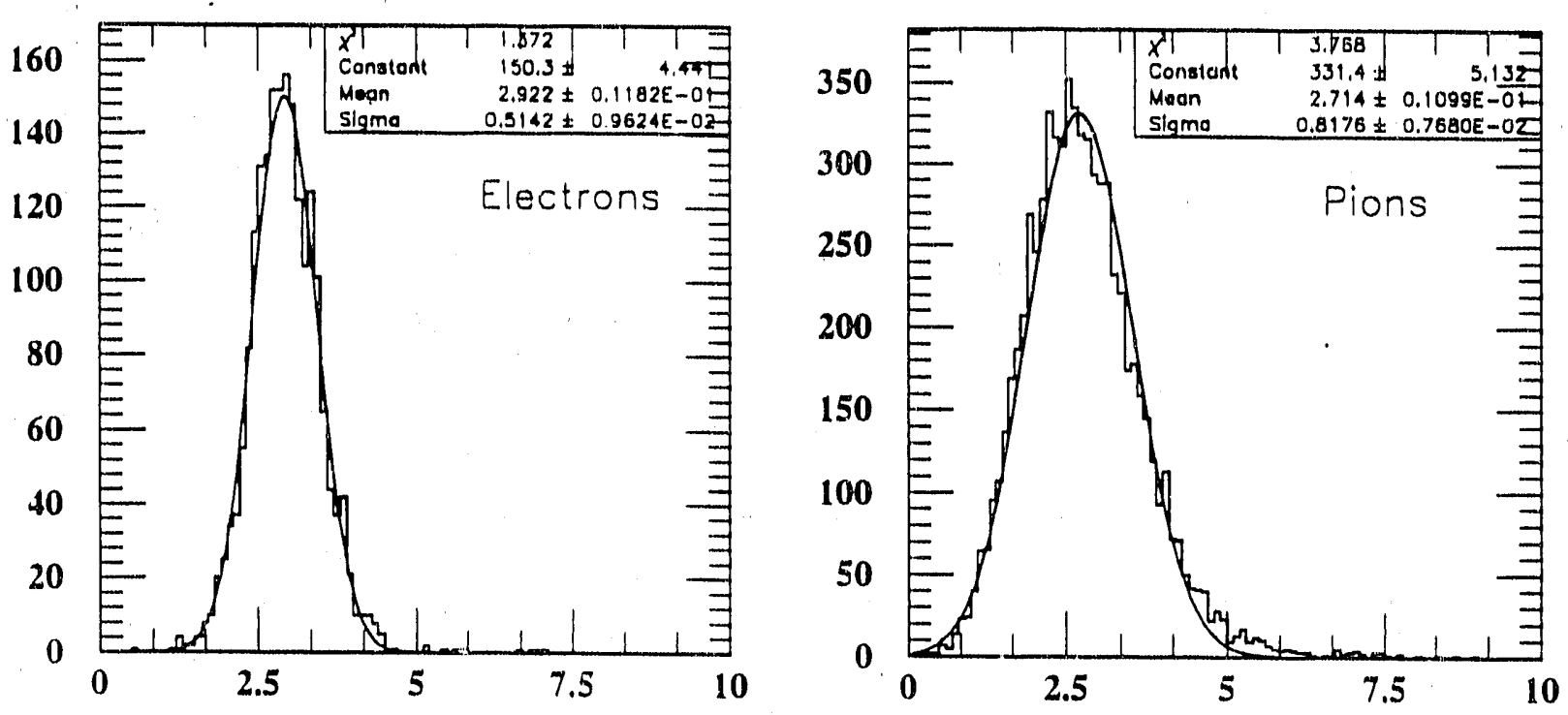

Figure 22: Response curves of a single tower $(5,16)$ to electrons and pions at $4.31 \mathrm{GeV} / \mathrm{c}$, from which the resolution values of the calorimeter are obtained.

the $\mathrm{e} / \pi$ response ratio is about 1.068 if the back leakage is considered as $6 \%$ as in the literature [12].

Figure 22 shows typical responses of the calorimeter to electron and $\pi$ beams. In this case, beams are aimed at the center of tower $(5,16)$. The resolution for electromagnetic shower is $28 \% / \sqrt{E}$ averaged among the calibrated towers while hadronic shower resolution is $50 \% / \sqrt{E}$. These resolution data will be put into a parametrization Monte Carlo program PROPHET simulating the calorimeter response.

\subsubsection{Trigger}

The design of the PCAL trigger system is that towers in every azimuthal segment are grouped into two fast analog sums on the side of the calorimeter, one for electromagnetic sections and one for hadronic sections. Each tower is weighted according to its geometry to form a analog sum of $E_{T}$ in that group. 32 analog sums are thus generated and being transmitted via fast cables to the counting house where trigger decisions are made before taking the event.

The trigger system of PCAL for the measurement of tranverse energy has been im- 


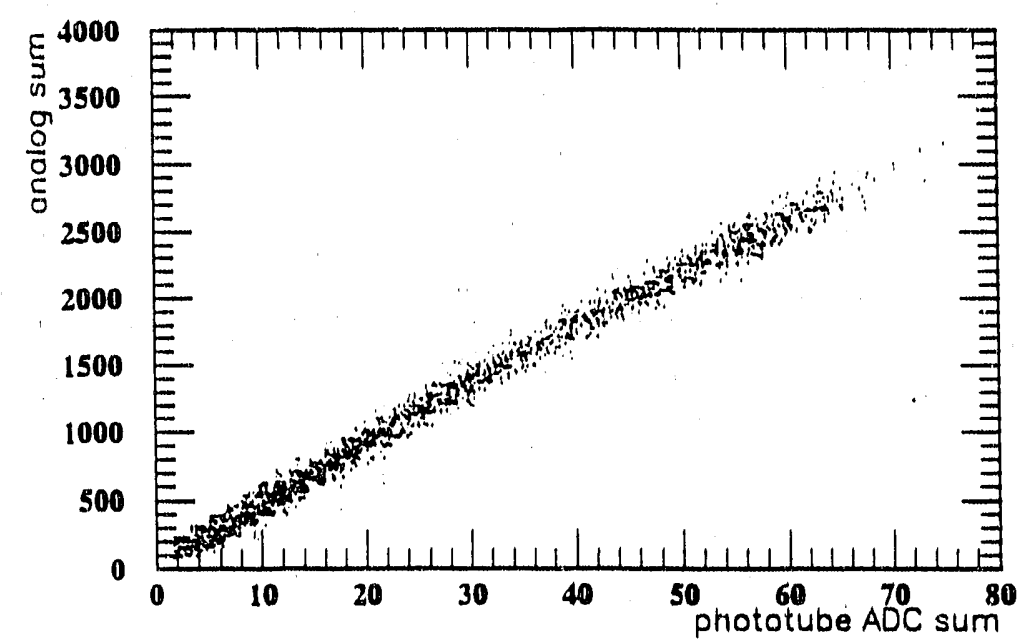

Figure 23: Trigger ananlog sum of PCAL $E_{T}$ versus the corresponding digital sum of all individual towers with proper geometrical weights.

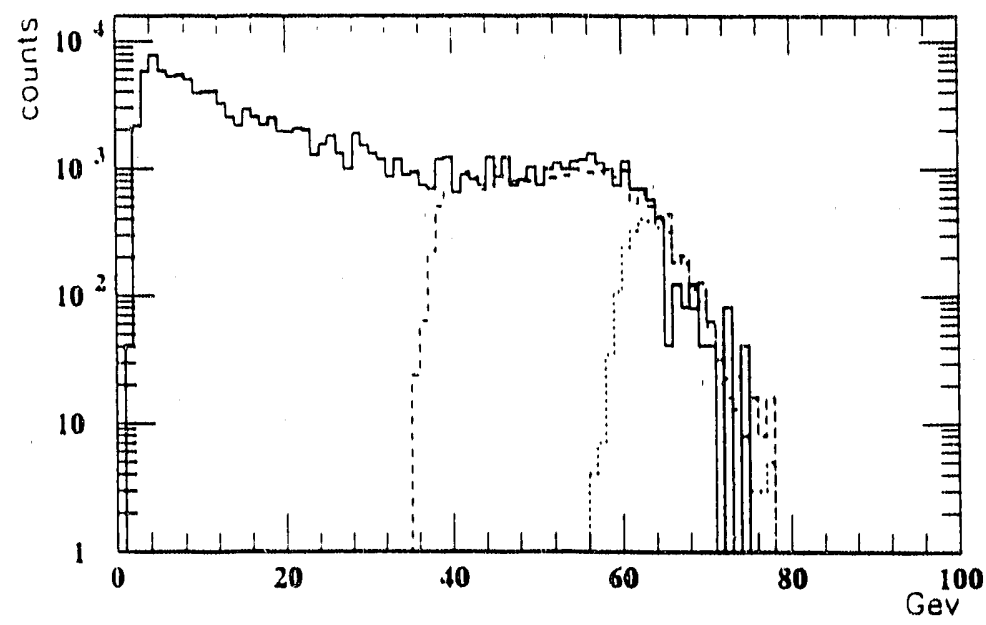

Figure 24: Online $E_{T}$ distribution showing triggering thresholds

proved before the ' 91 run. A number of errors in the trigger electronics had been corrected at the Pitt electronics shop and the system was tested before the run with a test pulse setup to insure its being operational during the run. In Figure 23 is the correlation between the analog sum of the total $E_{T}$ and the digital sum of all the phototube readouts. The linearity and width of the correlation shows the reliability of the trigger analog sum. Figure 24 indicates the positions of three $E_{T}$ trigger thresholds. Downscaling factors for these three levels are 41,8 and 1 . 


\subsubsection{Calorimeter Response simulation}

The program to simulate the calorimeter response is PROPHET, written first by Dagan and Oren for the Helios experiment at CERN. Instead of tracking every generated particle as the shower develops, PROPHET speeds up the calculations by parametrizing the shower development, both longitudinally and laterally. Such a parametrization is obtained from experimental results from calorimeter tests. It is, as expected, different from one type of calorimeter to another. The parameters used for PCAL simulation have been tuned for its showers size, e/ $\pi$ ratio and mip/e ratio etc. to best fit the detector response to various beams tested in the SEB run.

Parametrization is based that of R.Bock el al[13]. The starting point of the shower is calculated in the GEANT frame, based on various intercation cross-sections. The development of the electromagnetic showers is characterised by the radiation length of the media,

$$
d E=E * C * t^{\alpha-1} * e^{-\beta_{m t}} * d t
$$

where $C$ is the normalization constant, $t$ the depth in radiation length and $\alpha, \beta$ as parameters to fit.

Hadronic showers are parametrized with two parts, one with radiation length and one with absorption length of the media,

$$
d E=E * C *\left(w * t^{(a-1)} * e^{-b * t} * d t+(1-w) * s^{(c-1)} * e^{-d * s} * d t\right)
$$

where $w$ is the portion of the shower characterized by radiation length, $t$ in radiation length, $s$ in absorption length and $a, b, c, d$ are parameters to be tuned. The parameters usually have a logarithrnic energy dependence. To speed up cornputation, the above distributions as a function of $E, t$ and $s$ are calculated in the initialisation stage and stored in a table. 


\subsubsection{Data Analysis}

In Febuarary 1991, a week of running time was dedicated to transverse energy $\left(E_{T}\right)$ medm surements with participant calorimeter as the principal instrument. It was the first time this detector was used as a self-triggering device.

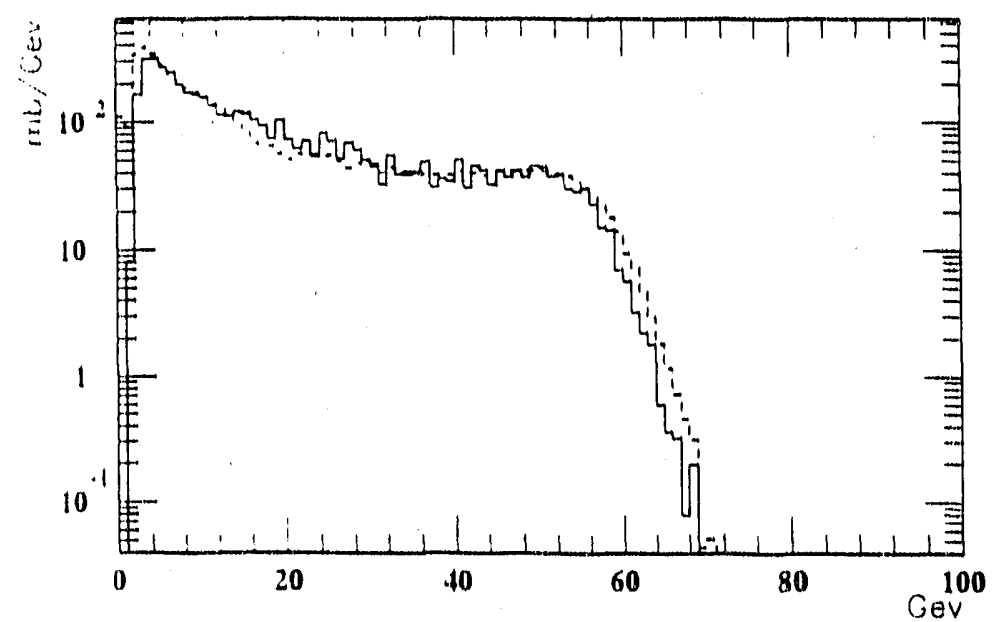

Figure 25: Raw $E_{T}$ distribution from the 1991 (solid curve) and 1990 (dashed curve) data. The two sets of data utilize two independent calibrations.

The calibration from the SEB run was tranfered back to Febuarary heavy ion data by comparing the ${ }^{60} \mathrm{Co}$ scans at the two times and then spread out to the entire detector using high multiplicity $(>100)$ heavy ion data, following the procedure in section 3.2 .2 . In Figure 25 is the raw transverse energy detected in the PCAL region. For consistency, also shown is the same spectrum from June 1990, with a set of totally independent calibration constants.

We are developing a mechanism to unfold raw data to real transverse energy distribution. A matrix $(M)$ is built to fold a certain $E_{T}$ distribution( $\alpha$ (from Monte Carlo) into simulated detector response $(D)$,

$$
M \alpha=D
$$

where $\alpha$ is an array of $8 \mathrm{~d} E_{T} / \mathrm{d} \eta$, binned as in Figure 26. $D$ is an array of 32 elements, each representing the sum of $E_{T}$ in towers with the size and same $\operatorname{depth}(\mathrm{a}$ ring). Each 


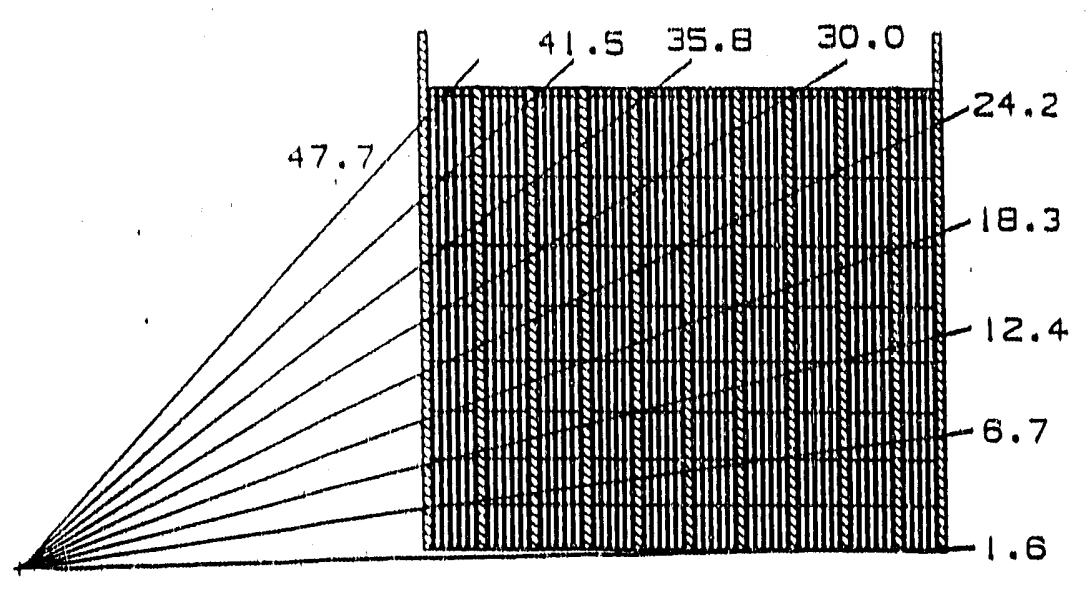

Figure 26: Diagram showing a side view of the upper half of PCAL indicating the pseudorapidity bins used in the analysis. The beam is incident from the left, and the target is located at the point marked $(+)$.

element of matrix $M$ is then naturally the percentage of $E_{T}$ deposited in a particular ring from a certain $\eta$ bin.

When applying the matrix to raw data(real detector response), a least $\chi^{2}$ approach is used. $\alpha$ is treated as a set of parameters to be tuned so that

$$
\chi^{2}(\alpha)=\sum_{i} \frac{\left(D_{i}-M_{i j} \alpha_{j}\right)}{\sigma_{i}^{2}}
$$

can be minimized. The fitted $\alpha$ is then expected to be the ieal $E_{T}$ distribution, By taking into consideration the depth information and tower to tower correlation due to shower spread, the model dependence on the Monte Carlo $E_{T}$ distribution is expected to be minimized.

To examine model (in)dependence of this method, FRITIOF and HIJET Monte Carlo are employed. $E_{T}$ distributions of both models are folded into simulated detector response by PROPHET, and two matrices are built accordingly. Figure 27a compares the original and unfolded $E_{T}$ distribution of FRITIOF, and shows the sensitivity of the unfolding method to different models. Figure 27b makes similiar comparison for HIJET.

In Figure 28 raw data are unfolded into total transverse energy distribution in PCAL's pseudorapidity region and compared with that of FRITIOF. It falls short of the simulation, 

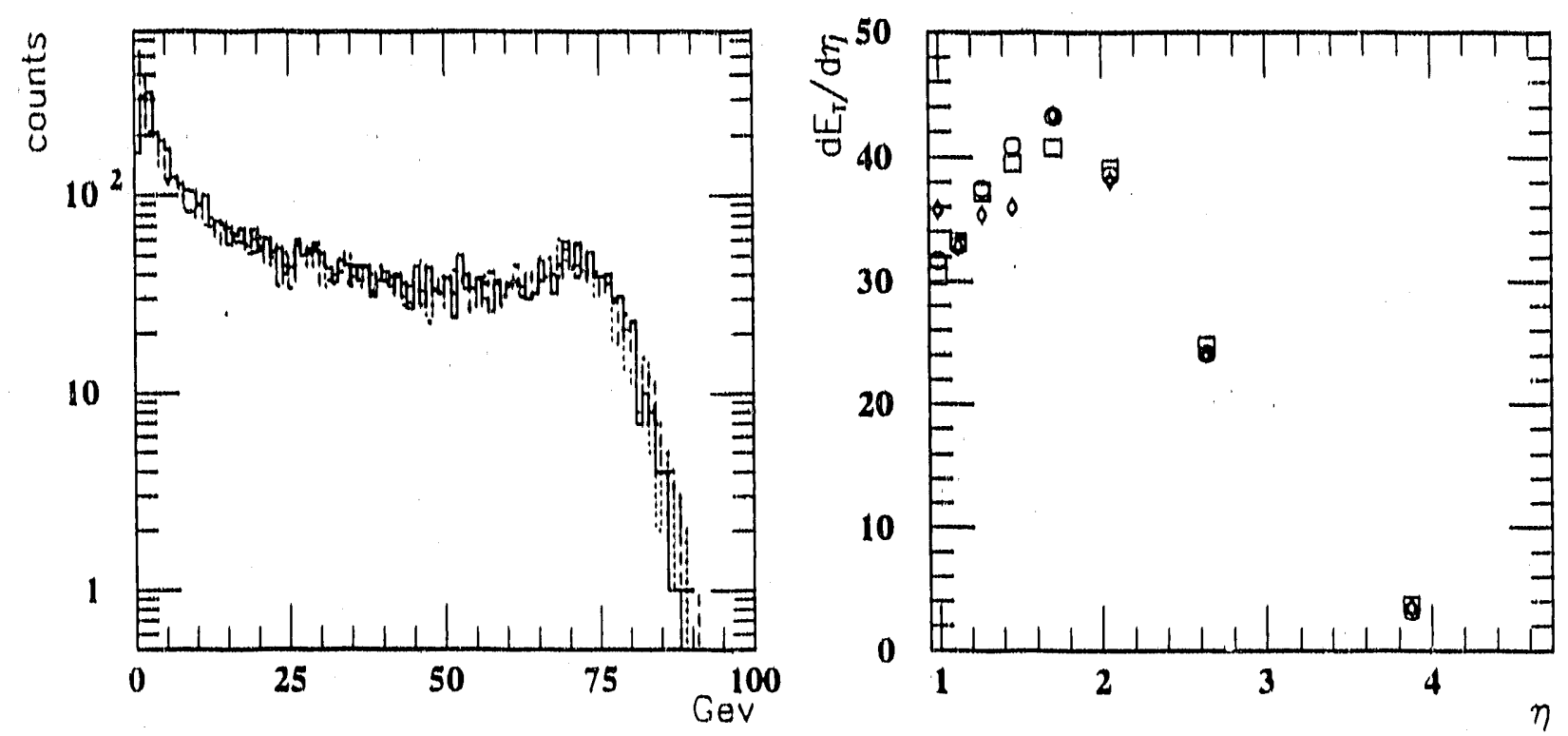

(a)
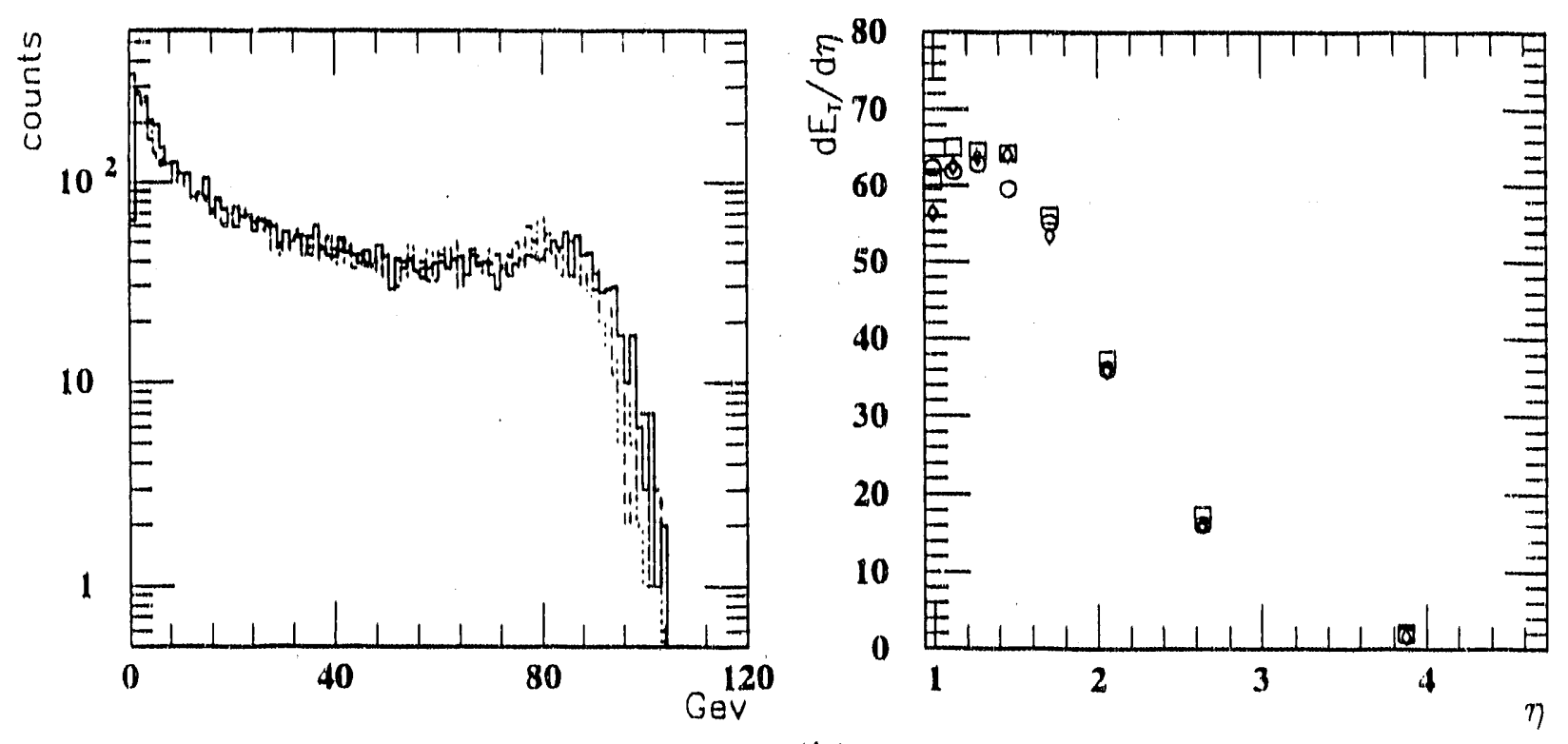

(b)

Figure 27: (a)FRITIOF simulation of the total $E_{T}$ distribution(solid curve on the left) and $\mathrm{d} E_{T} / \mathrm{d} \eta$ (squres on the right), compared with the corrected values obtained from the unfolding procedure using matrices derived from FRITIOF(dotted curve and cirles) and HIJET(dashed curve and diamonds). (b)The same test for HIJET 


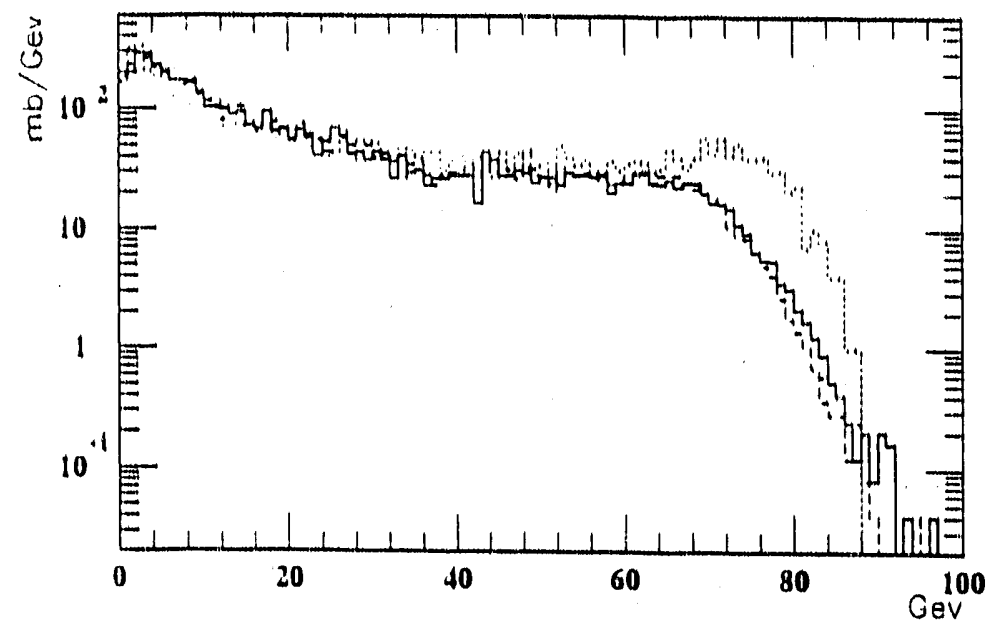

Figure 28: Total $E_{T}$ distribution obtained using correction matrices derived from FRITIOF(dashed) and HIJET(solid), Dotted curve is FRITIOF simulation

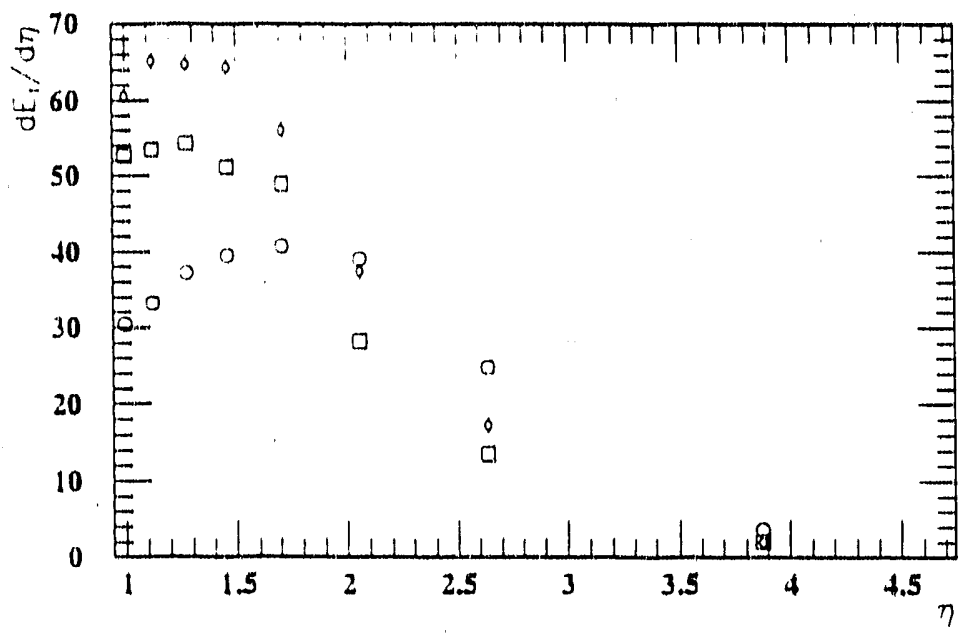

Figure 29: Values of $d E_{T} / d \eta$ obtained from the unfolding process using the matrix $M_{F R}$ obtained from FRITIOF (open squares), compared with the generated values from FRITIOF (circles) and HIJET (diamonds). 
and the cut-off is not as sharp as that of the simulation. The model independence of the unfolding is clearly shown when matrices generated both with FRITIOF and HIJET are applied to data and the results are almost identical.

Figure 29 compares the $d E_{T} / d \eta$ distribution to those of FRITIOF and HIJET. At large angle data are in between but at small angle data fali below both FRITIOF and HIJET.

\section{Personnel}

1. W. E. Cleland, principal investigator. Cleland's principal role in Experiment 814 has been in the area of organization of the trigger electronics. His efforts include planning of the trigger system, decisions regarding the purchase of commercial units for the trigger, supervision of the modification of existing electronics, and the design of new electronics which must be constructed. He also was responsible for the integration of the detector systems which make up the experiment. During the past year his major activity has been involvement in the analysis activities of the Pitt group.

2. E. Marcia Takagui. Takagui is a postdoctoral research associate who joined us in December 1989. She is working in the area of peripheral collisions and is presentiy concentrating on the the reconstruction of $2 p$ data, for which she is responsible. In addition she is system manager for our Silicon Graphics based computing system.

3. D. E. Kraus. Kraus is a part-time instructor at the University of Pittsburgh with considerable experience in experimental high energy physics. He has spent approxi- 
mately two-thirds of his time as a research associate for Experiment E814 during the years 1987-90. His principal technical responsibilities in E814 have been in studies of the behavior of photomultiplier tubes in the environment of heavy ion experiments; design, construction, calibration and maintenance of the forward and magnet scintillators; and development of a DC light source for use in the participant and beam calorimeters. Currently he is spending a small fraction (1/6) of his time participating in the analysis of the E814 data.

4. S. Voloshin. Voloshin is a theorist at the Moscow Engineering Institute who became interested in our multiplicity data and spent two months at Pitt as a visiting scientist. He worked with Jayananda on the analyis of the two particle correlation functions and was in important source of encouragement and theoretical guidance.

5. K. Jayananda. Jayananda was a graduate student who carried out his thesis research in central collisions. His first effort in this experiment, carried out during the summer and fall of 1987, were to analyze the data for albedo yields taken during the May 1987 test run. He then took responsibility for the design and commissioning of the silicon multiplicity detector, and helped to develop the summing electronics to integrate the information from the multiplicity detector into the second level trigger. His thesis, Appendix B of this report, was finished in October 1991, and included several studies of the charged multiplicity data. One of the more interesting topics he studied is the two particle correlation functions, made possible by the high statistical accuracy of our data. He now holds a faculty position at the University of Sri Jayewardenepura in Sri Lanka and plans to continue to collaborate on the two-particle correlation analysis.

6. Bo Yu. Bo Yu was a graduate student who carried out a thesis in high energy physics in instrumentation under the supervision of $\mathrm{V}$. Radeka of BNL. He was responsible for the detailed design of the prototype for DC1, a novel type of chamber using interpolative readout of cathode pads. He was then heavily involved in the design 
and testing of the prototype chamber and had followed through with the design, testing, and installation of the final chamber. He also designed the pad planes for DC2 and DC3. His thesis, finished in December 1991, was a study of drift chambers with interpolative cathode pad readouts. He currently holds a research position at Brookhaven National Laboratory.

7. U. Sonnadara. Upul Sonnadara is a graduate student who has been working with us since ine summer of 1988 . He passed the comprehensive examination in May, 1989. He has helped with the assembly and calibration of the forward scintillation counters, and he has written monitor programs on the data-acquisition VAX in order to monitor their performance. He has taken on the responsibility for the calibration of the forward and magnet scintillators. He is heavily involved in the peripheral data analysis and was the major author on the 1p paper incluaed in Appendix A. He plans to continue his thesis research with an analysis of the $1 \mathrm{n}$ data.

8. Z. Zhang. Zhang is a graduate student who joined our group in April, 1989, having passed the comprehensive exam in May, 1987. His principal responsibility is for the data taken with the participant calorimeter, so during the past year much of his effort has been spent on the calibration and gain matching of this device, including a and a study of the triggering electronics. In addition Zhang built the trigger monitoring workstation based on expert systems techniques, installed in E814 before the June 1990 run, and he continues to maintain this device. He plans to write a thesis in the area of central collisions, concentrating on data from the participant calorimeter.

9. Tomas Joyce. Joyce is an undergraduate engineering student who has been working with our research group on a part time basis since the spring of 1991 . He has carried out a number of data analysis tasks, including studies of the gains of photomultiplier tubes required for the peripheral analysis and studies of neutron calorimeter using calibration data taken with a proton beam.

10. Brian Lopresti. Lopresti is an undergraduate physics major who has been working 
with our group on a part time basis since the spring of 1991 . He has been helping with analysis tasks, including studies of the timing properties of the time of flight counters installed in the experiment in 1990. He is currently involved in the analysis of peripheral collisions with $\alpha$ particles in the final state.

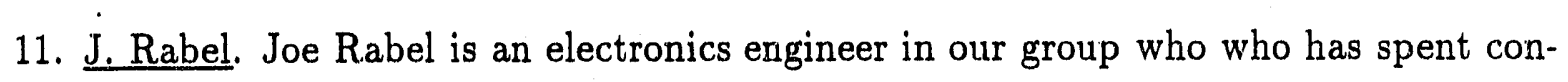
siderable effort on the E814 electronics during the past several years. His expertise in digital logic has been invaluable in carrying out the design and testing of the CAMAC modules needed for our trigger processor. Mr. Rabel also maintains the electronics modules which are used in E814, including the large number of current monitors used for calibrating the calorimeters. Currently he is involved in work connected with our RHIC R\&D project, in which field programmable gate arrays are being evaluated for their usefulness in triggering applications. 


\section{References}

[1] B. Bassalleck, et al. Z. Phys C 38 (1988) 45

[2] J. Barrette et al. Phys. Rev. Letters 64 (1990) 1219.

[3] J. Barrette et al. Phys. Rev. C 41 (1990) 1512.

[4] J. Barrette et al. Phys. Letters B252 (1990) 550.

[5] J. Barrette et al. Phys. Rev. C, to be published.

[6] J. Barrette et al. "Excitation Energy Distribution of Relativistic ${ }^{28} \mathrm{Si}$ in Electromagnetic Dissociation into $\mathbf{p}+{ }^{27} \mathbf{A l}$ ", submitted for publication in Phys. Rev. C Dec 1991.

[7] J. Barrette et al. "Charged particle multiplicity in ${ }^{28} \mathrm{Si}+\mathrm{Al}, \mathrm{Cu}$ and $\mathrm{Pb}$ reactions at $E_{l a b}=14.6 \mathrm{GeV} /$ nucleon", submitted for publication in Phys. Rev. C Nov 1991.

[8] J. Fischer at al., IEEE Trans. Nucl. Sci., NS-37 (1990).

[9] R. Debbe at al., IEEE Trans. Nucl. Sci., NS-37, (1990).

[10] Bo Yu "Gas Proportional Detectors with Interpolating Cathode Pad Readout for High Track Multiplicities", Ph.D. thesis, University of Pittsburgh, December 1991

[11] J. Lissauer and H. Takai, Phys, Rev. C 41 (1990) 2410.

[12] M.G. Catanesi at al, Nucl. Inst. and Methods A260(1987) 43-54

[13] R.K. Bock at al, Nucl. Inst. and Nethods 186(1981) 533-539

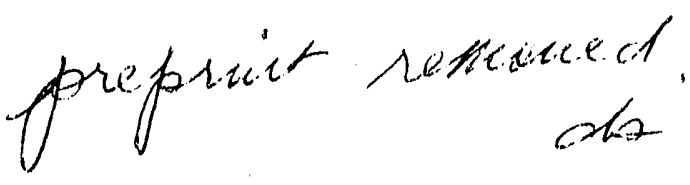




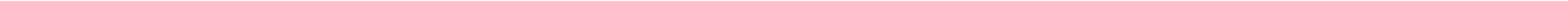




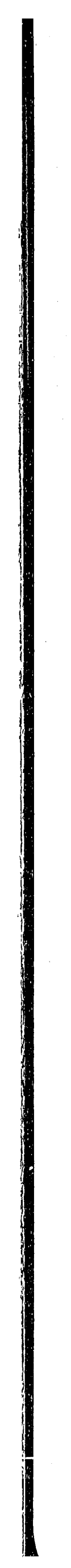

